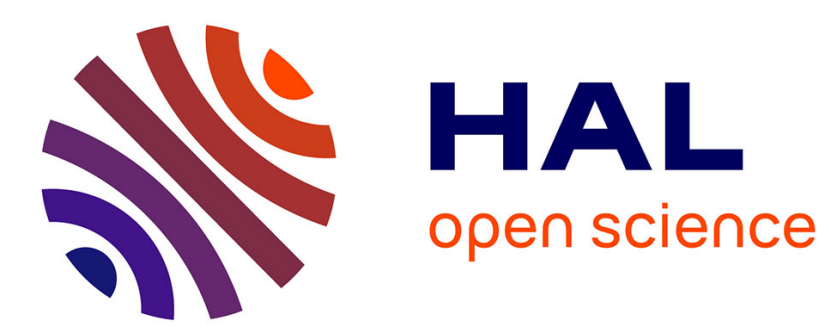

\title{
Control and Stabilization of the Nonlinear Schroedinger Equation on Rectangles
}

\author{
Lionel Rosier, Bing-Yu Zhang
}

\section{To cite this version:}

Lionel Rosier, Bing-Yu Zhang. Control and Stabilization of the Nonlinear Schroedinger Equation on Rectangles. Mathematical Models and Methods in Applied Sciences, 2010, 20 (12), pp.2293-2347. 10.1142/S0218202510004933 . hal-00453074

\section{HAL Id: hal-00453074 https://hal.science/hal-00453074}

Submitted on 3 Feb 2010

HAL is a multi-disciplinary open access archive for the deposit and dissemination of scientific research documents, whether they are published or not. The documents may come from teaching and research institutions in France or abroad, or from public or private research centers.
L'archive ouverte pluridisciplinaire HAL, est destinée au dépôt et à la diffusion de documents scientifiques de niveau recherche, publiés ou non, émanant des établissements d'enseignement et de recherche français ou étrangers, des laboratoires publics ou privés. 


\title{
Control and Stabilization of the Nonlinear Schrödinger Equation on Rectangles
}

\author{
Lionel Rosier * $\quad$ Bing-Yu Zhang ${ }^{\dagger}$
}

February 3, 2010

\begin{abstract}
This paper studies the local exact controllability and the local stabilization of the semilinear Schrödinger equation posed on a product of $n$ intervals $(n \geq 1)$. Both internal and boundary controls are considered, and the results are given with periodic (resp. Dirichlet or Neumann) boundary conditions. In the case of internal control, we obtain local controllability results which are sharp as far as the localization of the control region and the smoothness of the state space are concerned. It is also proved that for the linear Schrödinger equation with Dirichlet control, the exact controllability holds in $H^{-1}(\Omega)$ whenever the control region contains a neighborhood of a vertex.
\end{abstract}

Key words. Schrödinger equation, Bourgain spaces, exact boundary controllability, exact internal controllability, exponential stabilization

\section{Introduction}

The control of the Schrödinger equation has received a lot of attention in the last decades. (See e.g. [52] for an excellent review of the contributions up to 2003). Significant progresses have been made for the linear Schrödinger equation on its controllability and stabilizability properties (see [21, 24, 31, 36, 37, 38, 40, 43] for control issues, and [3, 11, 12, 39, 51] for Carleman estimates and their applications to inverse problems). For the control of the so-called bilinear Schrödinger equation, in which the bilinear term is linear in both

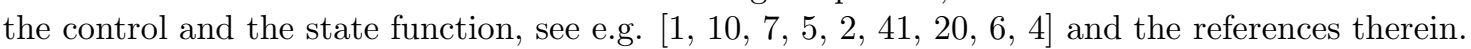

By contrast, the study of the nonlinear Schrödinger equation is still at its early stage. Recently, Illner, Lange and Teismann [19, 20] considered the internal controllability of the nonlinear Schrödinger equation posed on a finite interval with periodic boundary conditions:

$$
i u_{t}+u_{x x}+f(u)=i a(x) h(x, t) .
$$

In (1), a denotes a smooth real function which is strictly supported in $\mathbb{T}$, the one-dimensional torus. They

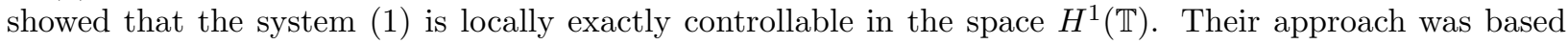
on the well-known Hilbert Uniqueness Method (HUM) and Schauder's fixed point theorem. Later, Lange and Teismann [25] considered internal control for the nonlinear Schrödinger equation (11) posed on a finite interval with the homogeneous Dirichlet boundary conditions

$$
u(0, t)=u(\pi, t)=0
$$

and established local exact controllability of the system (1)-(2) in the space $H_{0}^{1}(0, \pi)$ around a special ground state of the system. Their approach was mainly based upon HUM and the implicit function theorem. Dehman, Gérard and Lebeau [13 studied the internal control and stabilization of a class of defocusing nonlinear Schrödinger equations posed on a two-dimensional compact Riemannian manifold $M$ without boundary

$$
i u_{t}+\Delta u+f(u)=i a(x) h(x, t) .
$$

They demonstrated, in particular, that the system is (semiglobally) exactly controllable and stabilizable in the space $H^{1}(M)$ assuming that the Geometric Control Condition and some unique continuation condition are satisfied.

\footnotetext{
*Institut Élie Cartan, UMR 7502 UHP/CNRS/INRIA, B.P. 239, 54506 Vandouvre-lès-Nancy Cedex, France. (rosier@iecn.u-nancy.fr)

${ }^{\dagger}$ Department of Mathematical Sciences, University of Cincinnati, Cincinnati, Ohio 45221, USA. (bzhang@math.uc.edu)
} 
Recently, the authors proved in 46 that the cubic Schrödinger equation on the torus $\mathbb{T}$ with a localized control

$$
i u_{t}+u_{x x}+\lambda|u|^{2} u=i a(x) h(x, t), \quad x \in \mathbb{T}
$$

is locally exactly controllable in $H^{s}(\mathbb{T})$ for all $s \geq 0$ (hence, in $L^{2}(\mathbb{T})$ ). Inspired by the work of Russell-Zhang in [48], the method of proof combined the momentum approach and Bourgain analysis. In the same paper, the local stabilization by the feedback law $h=a(x) u(x, t)$ was established by applying the contraction mapping theorem in some Bourgain space. Finally, similar results were obtained with Dirichlet (resp. Neumann) homogeneous boundary conditions thanks to an extension argument. More recently, Laurent has shown in 28] that the system (3) is semiglobally exactly controllable and stabilizable. The same result has also been derived by Laurent in 29] for certain manifolds of dimension 3, including $\mathbb{T}^{3}, S^{3}$, and $S^{2} \times S^{1}$. The propagation of compactness and regularity proved in [28, 29] plays a crucial role in the derivation of the stabilization results in these papers. See also [30] for another application of these ideas to the semiglobal stabilization of the periodic Korteweg-de Vries equation.

In addition, the authors considered in [47] the following nonlinear Schrödinger equation

$$
i u_{t}+\Delta u+\lambda|u|^{2} u=0
$$

posed on a bounded domain $\Omega$ in $\mathbb{R}^{n}$ with either the Dirichlet boundary conditions or the Neumann boundary conditions. They showed that if

$$
s>\frac{n}{2}
$$

or

$$
0 \leq s<\frac{n}{2} \text { with } 1 \leq n<2+2 s
$$

or

$$
s=0,1 \text { with } n=2,
$$

then the systems with control inputs acting on the whole boundary of $\Omega$ are locally exactly controllable in the classical Sobolev space $H^{s}(\Omega)$ around any smooth solution of the Schrödinger equation.

The aim of this paper is to extend the results of [46] to any dimension. More precisely, we shall assume that the spatial variable lives in the rectangle

$$
\Omega=\left(0, l_{1}\right) \times \cdots \times\left(0, l_{n}\right) .
$$

We shall investigate the control properties of the semilinear Schrödinger equation

$$
i u_{t}+\Delta u+\lambda|u|^{\alpha} u=i a(x) h(x, t)
$$

where $\lambda \in \mathbb{R}$ and $\alpha \in 2 \mathbb{N}^{*}$, by combining new linear controllability results in the spaces $H^{s}(\Omega)$ with Bourgain analysis. Let us briefly review the results proved in this paper.

The internal controllability of the linear Schrödinger equation on $\mathbb{T}^{n}$

$$
i u_{t}+\Delta u=i a(x) h(x, t), \quad x \in \mathbb{T}^{n}, t \in(0, T)
$$

is established in $H^{s}\left(\mathbb{T}^{n}\right)$ for any $s \geq 0$ and any function $a \not \equiv 0$. (Note that the Geometric Control Condition is not required.) It is derived from a well-known result in $L^{2}\left(\mathbb{T}^{n}\right)$, due to Jaffard [21] when $n=2$ and Komornik [23] for any $n \geq 2$, by an argument allowing to shift the (state and control) space from $L^{2}\left(\mathbb{T}^{n}\right)$ to $H^{s}\left(\mathbb{T}^{n}\right)$. In particular, the exact controllability in $H^{s}\left(\mathbb{T}^{n}\right)$ will require a control input $h \in L^{2}\left(0, T ; H^{s}\left(\mathbb{T}^{n}\right)\right)$. Similar results with Dirichlet or Neumann homogeneous boundary conditions are deduced by using the extension argument from 46 .

The boundary controllability of the linear Schrödinger equation is considered both with Dirichlet control

$$
u=1_{\Gamma_{0}} h(x, t)
$$

and with Neumann control

$$
\frac{\partial u}{\partial \nu}=1_{\Gamma_{0}} h(x, t)
$$

In (6) and in (7), $\Gamma_{0}$ denotes an open set in $\partial \Omega$. For the Dirichlet control, we shall prove that in any dimension $n \geq 2$ the exact controllability holds in $H^{-1}(\Omega)$ whenever $\Gamma_{0}$ is a neighborhood of a vertex 
of $\Omega$. The observability inequality for this (arbitrarily small) control region is actually derived from the corresponding observability inequality for internal control by multiplier techniques.

For the Neumann control, the exact controllability in $L^{2}(\Omega)$ is obtained in any dimension when $\Gamma_{0}$ is a side. Finally, the results with Dirichlet (resp Neumann) boundary control are extended to any Sobolev space $H^{s}(\Omega)$ with $s<1 / 2$ (resp. $s<1$ ) by considering control inputs more regular in time, namely $h \in H^{\frac{s+1}{2}}\left(0, T ; L^{2}(\partial \Omega)\right)$ (resp. $\left.h \in H^{\frac{s}{2}}\left(0, T ; L^{2}(\partial \Omega)\right)\right)$.

The extension of the above exact controllability results to the semilinear Schrödinger equation

$$
i u_{t}+\Delta u+\lambda|u|^{\alpha} u=i a(x) h(x, t)
$$

is performed on the basis of Bourgain analysis. The needed linear and multilinear estimates are combined with a fixed-point argument to produce local exact controllability results. Sharp results (for the support of the control input) are given for the internal control. Boundary controllability results are derived from those established for the linear equation with the aid of estimates in Bourgain spaces of solutions of boundary-value problems with boundary terms given by HUM.

Finally, the local exponential stabilization with an internal feedback law is proved by following the same approach as in 46 .

The paper is organized as follows. The controllability results for the linear Schrödinger equation are collected in Section 2. Section 3 is devoted to the controllability of the semilinear equations. Section 4 deals with the internal stabilization issue. Multilinear estimates for nonlinearities of the form $u^{\alpha_{1}} \bar{u}^{\alpha_{2}}$ are established in Appendix.

\section{Linear systems}

\section{$2.1 \quad$ Internal control}

We first consider the linear open loop control system for the Schrödinger equation posed on $\mathbb{T}^{n}:=(-\pi, \pi)^{n}$ with periodic boundary conditions:

$$
i u_{t}+\Delta u=i G h:=i a(x) h(x, t), \quad u(x, 0)=u_{0}(x),
$$

where $a \in C^{\infty}\left(\mathbb{T}^{n}\right)$ is a given smooth real-valued function and $h=h(x, t)$ is the control input.

We denote by $H^{s}\left(\mathbb{T}^{n}\right)$ the Sobolev space of the functions $u$ defined on the torus $\mathbb{T}^{n}$ (i.e. defined on $\mathbb{R}^{n}$ and periodic of period $2 \pi$ with respect to each variable $x_{i}$ ) for which the $H^{s}$ norm

$$
\|u\|_{s}=\left\|(1-\Delta)^{s / 2} u\right\|_{L^{2}\left(\mathbb{T}^{n}\right)}
$$

is finite.

We first establish an internal observability inequality for the solution $v(t)=W(t) v_{0}$ of

$$
\left\{\begin{array}{l}
i v_{t}+\Delta v=0 \quad(x, t) \in \mathbb{T}^{n} \times \mathbb{R}, \\
v(0)=v_{0}
\end{array}\right.
$$

Proposition 2.1 (Observability inequality in $H^{-s}\left(\mathbb{T}^{n}\right)$ ) Let $a \in C^{\infty}\left(\mathbb{T}^{n}\right)$ with $a \neq 0$ and $T>0$. Then for any $s \geq 0$ there exists a constant $c>0$ such that for any solution $v$ of (10) with $v_{0} \in H^{-s}\left(\mathbb{T}^{n}\right)$, it holds

$$
\left\|v_{0}\right\|_{-s}^{2} \leq c \int_{0}^{T}\|a v(t)\|_{-s}^{2} d t .
$$

Proof. We proceed in several steps.

Step 1. Assume that $s=0$, and let

$$
\omega=\left\{x \in(-\pi, \pi)^{n} ;|a(x)|>\|a\|_{L^{\infty}\left(\mathbb{T}^{n}\right)} / 2\right\} .
$$

Then, by 24, Lemma 8.9], there exists some positive constant $c$ such that for any square-summable sequence $\left(c_{k}\right)_{k \in \mathbb{Z}^{n} \backslash\{0\}}$ we have

$$
\sum_{k \neq 0}\left|c_{k}\right|^{2} \leq c \int_{0}^{T} \int_{\omega}\left|\sum_{k \neq 0} c_{k} e^{i\left(k \cdot x-|k|^{2} t\right)}\right|^{2} d x d t .
$$


The result is still valid when the set of indices is changed into $\mathbb{Z}^{n}$ by [24, Proposition 8.4]. This yields (11) when $s=0$.

Step 2. We prove the weaker inequality

$$
\left\|v_{0}\right\|_{-s}^{2} \leq c\left(\int_{0}^{T}\|a v(t)\|_{-s}^{2} d t+\left\|v_{0}\right\|_{-s-1}^{2}\right)
$$

by contradiction. If $(13)$ is false, then there exists a sequence $\left\{v_{j}\right\}$ of solutions of $(10)$ in $C\left([0, T] ; H^{-s}\left(\mathbb{T}^{n}\right)\right)$ such that

$$
1=\left\|v_{j}(0)\right\|_{-s}^{2} \geq j\left(\int_{0}^{T}\left\|a v_{j}(t)\right\|_{-s}^{2} d t+\left\|v_{j}(0)\right\|_{-s-1}^{2}\right) .
$$

Since $v_{j}$ is bounded in $L^{\infty}\left([0, T] ; H^{-s}\left(\mathbb{T}^{n}\right)\right)$ and $\left(v_{j}\right)_{t}$ is bounded in $L^{\infty}\left([0, T] ; H^{-s-2}\left(\mathbb{T}^{n}\right)\right)$ by $(10)$, we infer from Aubin's lemma that, for a subsequence again denoted by $\left\{v_{j}\right\}$, we have for $j \rightarrow \infty$

$$
\begin{cases}v_{j} \rightarrow v & \text { in } L^{\infty}\left([0, T] ; H^{-s}\left(\mathbb{T}^{n}\right)\right) \quad \text { weak * } \\ v_{j} \rightarrow v & \text { in } C\left([0, T] ; H^{r}\left(\mathbb{T}^{n}\right)\right) \quad \forall r<-s\end{cases}
$$

where $v \in C_{w}\left([0, T] ; H^{-s}\left(\mathbb{T}^{n}\right)\right)$ is a solution of (10). In particular, $v_{j}(0) \rightarrow v(0)$ in $H^{r}\left(\mathbb{T}^{n}\right)$ for any $r<-s$. Since $v_{j}(0) \rightarrow 0$ in $H^{-s-1}\left(\mathbb{T}^{n}\right)$ by $(14)$, we conclude that $v \equiv 0$. Let $w_{j}=(1-\Delta)^{-s / 2} v_{j}$. Then $w_{j} \in$ $L^{\infty}\left([0, T] ; L^{2}\left(\mathbb{T}^{n}\right)\right)$ and

$$
\begin{cases}w_{j} \rightarrow 0 & \text { in } L^{\infty}\left([0, T] ; L^{2}\left(\mathbb{T}^{n}\right)\right) \quad \text { weak * } \\ w_{j} \rightarrow 0 & \text { in } C\left([0, T] ; H^{r}\left(\mathbb{T}^{n}\right)\right) \quad \forall r<0 .\end{cases}
$$

Let us split $a w_{j}$ into

$$
a w_{j}=(1-\Delta)^{-s / 2}\left(a v_{j}\right)-(1-\Delta)^{-s / 2}\left[a,(1-\Delta)^{s / 2}\right] w_{j} .
$$

As the pseudodifferential operator $\left[a,(1-\Delta)^{s / 2}\right]$ maps continuously $H^{r}\left(\mathbb{T}^{n}\right)$ into $H^{r-s+1}\left(\mathbb{T}^{n}\right)$, we have that

$$
(1-\Delta)^{-s / 2}\left[a,(1-\Delta)^{s / 2}\right] w_{j} \rightarrow 0 \quad \text { in } C\left([0, T] ; H^{r}\left(\mathbb{T}^{n}\right)\right) \text { for any } r<1 .
$$

Therefore, using (14) and (15), we obtain that

$$
a w_{j} \rightarrow 0 \text { in } L^{2}\left([0, T] ; L^{2}\left(\mathbb{T}^{n}\right)\right)
$$

Clearly, $w_{j}$ satisfies also the linear Schrödinger equation (10), so we infer from the observability inequality (11) established for $s=0$ that

$$
w_{j}(0) \rightarrow 0 \text { in } L^{2}\left(\mathbb{T}^{n}\right)
$$

It follows that $v_{j}(0)=(1-\Delta)^{s / 2} w_{j}(0) \rightarrow 0$ in $H^{-s}\left(\mathbb{T}^{n}\right)$, contradicting the fact that $\left\|v_{j}(0)\right\|_{-s}=1$ for all $j$.

Step 3. We prove (11) by contradiction. If (11) is false, there exists a sequence $\left\{v_{j}\right\}$ of solutions of (10) in $C\left([0, T] ; H^{-s}\left(\mathbb{T}^{n}\right)\right)$ such that

$$
1=\left\|v_{j}(0)\right\|_{-s}^{2} \geq j \int_{0}^{T}\left\|a v_{j}(t)\right\|_{-s}^{2} d t \quad \forall j \geq 0 .
$$

Extracting a subsequence if needed, we may assume that

$$
\begin{array}{ll}
v_{j} \rightarrow v & \text { in } L^{\infty}\left([0, T] ; H^{-s}\left(\mathbb{T}^{n}\right)\right) \quad \text { weak } * \\
v_{j} \rightarrow v & \text { in } C\left([0, T] ; H^{r}\left(\mathbb{T}^{n}\right)\right) \quad \forall r<-s
\end{array}
$$

for some solution $v \in C_{w}\left([0, T] ; H^{-s}\left(\mathbb{T}^{n}\right)\right)$ of $(10)$, where $C_{w}\left([0, T] ; H^{-s}\left(\mathbb{T}^{n}\right)\right.$ denotes the space of weakly sequentially continuous functions from $[0, T]$ to $H^{-s}\left(\mathbb{T}^{n}\right)$ (see [35, Lemme 8.1]). Clearly, $a v_{j} \rightarrow a v$ in $L^{\infty}\left([0, T] ; H^{-s}\left(\mathbb{T}^{n}\right)\right)$ weak $*$ which, combined to $(16)$, yields av $\equiv 0$. An application of Holmgren theorem (see e.g. [18. Theorem 8.6.5]) gives $v \equiv 0$. On the other hand, (18) gives $v_{j}(0) \rightarrow 0$ in $H^{-s-1}\left(\mathbb{T}^{n}\right)$. It then follows from (13) that $v_{j}(0) \rightarrow 0$ in $H^{-s}\left(\mathbb{T}^{n}\right)$, and this contradicts 16 .

Applying HUM [34] with $L^{2}\left(\mathbb{T}^{n}\right)$ as pivot space, we infer from Proposition 2.1 the following internal controllability of the linear Schrödinger equation in $H^{s}\left(\mathbb{T}^{n}\right)$. 
Theorem 2.2 Let $T>0$ and $s \geq 0$ be given. Then for any $\left(u_{0}, u_{1}\right) \in H^{s}\left(\mathbb{T}^{n}\right) \times H^{s}\left(\mathbb{T}^{n}\right)$ there exists a control $h \in L^{2}\left([0, T] ; H^{s}\left(\mathbb{T}^{n}\right)\right)$ such that the system (9) admits a unique solution $u \in C\left([0, T] ; H^{s}\left(\mathbb{T}^{n}\right)\right)$ satisfying $u(T)=u_{1}$. Moreover, we can define a bounded operator

$$
\Phi: H^{s}\left(\mathbb{T}^{n}\right) \times H^{s}\left(\mathbb{T}^{n}\right) \rightarrow L^{2}\left([0, T] ; H^{s}\left(\mathbb{T}^{n}\right)\right)
$$

such that for any $\left(u_{0}, u_{1}\right) \in H^{s}\left(\mathbb{T}^{n}\right) \times H^{s}\left(\mathbb{T}^{n}\right)$ it holds

$$
W(T) u_{0}+\int_{0}^{T} W(T-\tau)\left(G\left(\Phi\left(u_{0}, u_{1}\right)\right)\right)(\cdot, \tau) d \tau=u_{1} .
$$

The (small) control region is represented in Figure 1. Trapped rays are drawn to mean that the wave equation fails to be controllable with such control regions.

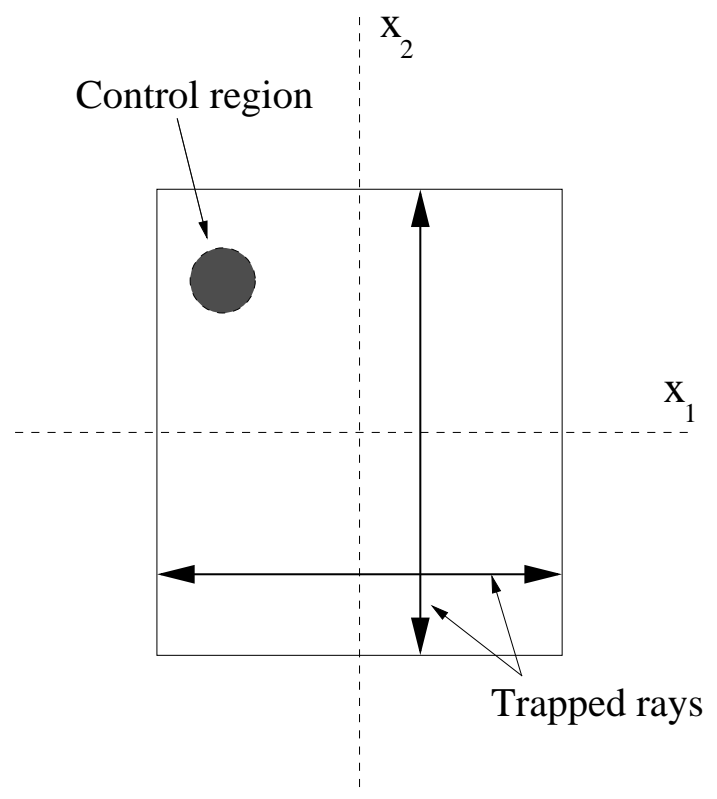

Figure 1: Internal control of the Schrödinger equation.

\subsection{Boundary control}

In this section $\Omega=(0, \pi)^{n}$, and $\Gamma_{0}$ denotes an open set in $\partial \Omega$.

\subsubsection{Dirichlet boundary control}

We first adopt the following definition.

Definition 2.3 The open set $\Gamma_{0} \subset \partial \Omega$ is called a Dirichlet control domain if given any $u_{0}, u_{1} \in H^{-1}(\Omega)$ and any time $T>0$, one may find a control $h \in L^{2}\left(0, T ; L^{2}\left(\Gamma_{0}\right)\right)$ such that the solution $u=u(x, t)$ of

$$
\begin{cases}i u_{t}+\Delta u=0 & \text { in } \Omega \times(0, T) \\ u=1_{\Gamma_{0}} h(x, t) & \text { on } \partial \Omega \times(0, T) \\ u(0)=u_{0} & \end{cases}
$$

satisfies $u(T)=u_{1}$.

The following result provides Dirichlet control domains which are arbitrary small in any dimension $n \geq 2$. Note that the wave equation fails to be controllable with such control domains.

Theorem 2.4 Let $\Omega=(0, \pi)^{n}$, and let $\Gamma_{0} \subset \partial \Omega$ be any open set containing a vertex of $\partial \Omega$. Then $\Gamma_{0}$ is a Dirichlet control domain. 


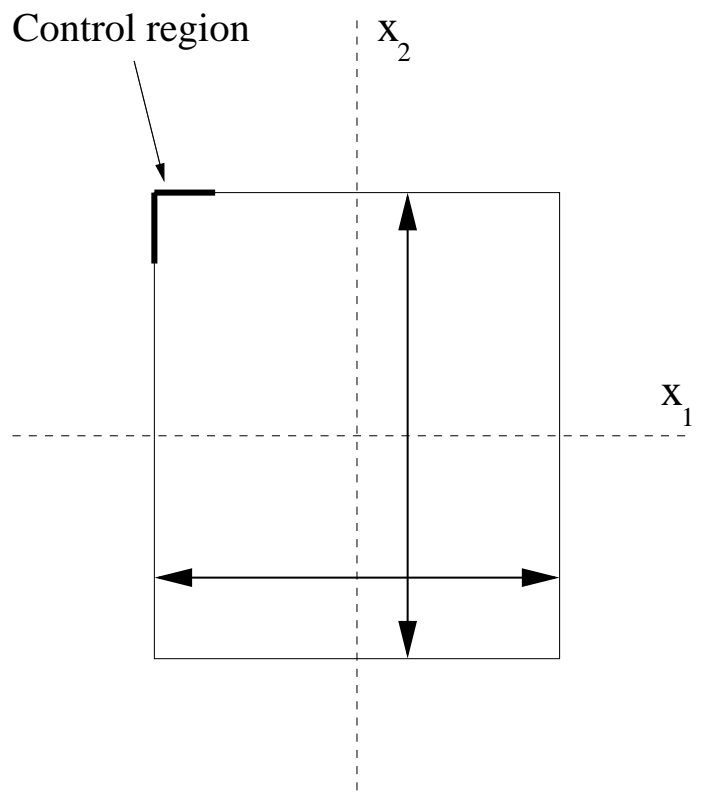

Figure 2: Boundary control of the Schrödinger equation.

By Dolecki-Russell test of controllability (or HUM), Theorem 2.4 is a direct consequence of the following boundary observability result for the system

$$
\begin{cases}i v_{t}+\Delta v=0 & \text { in } \Omega \times(0, T) \\ v=0 & \text { on } \partial \Omega \times(0, T) \\ v(0)=v_{0} . & \end{cases}
$$

Proposition 2.5 Assume that the (open) control region $\Gamma_{0} \subset \partial \Omega$ contains a vertex of $\partial \Omega$. Then for every $T>0$, there exists a constant $c>0$ such that

$$
\left\|\nabla v_{0}\right\|_{L^{2}(\Omega)}^{2} \leq c \int_{0}^{T} \int_{\Gamma_{0}}\left|\frac{\partial v}{\partial \nu}\right|^{2} d \sigma d t
$$

for any solution $v$ of (21) with $v_{0} \in H_{0}^{1}(\Omega)$.

Proof. We proceed in several steps.

Step 1. First, we prove an observability inequality in $H_{0}^{1}(\Omega)$ with an internal observation in an arbitrary subdomain of $\Omega$.

Lemma 2.6 Let $\omega \subset \Omega$ be an arbitrary nonempty open set. Then there exists a constant $c>0$ such that

$$
\left\|\nabla v_{0}\right\|_{L^{2}(\Omega)}^{2} \leq c \int_{0}^{T} \int_{\omega}|\nabla v(x, t)|^{2} d x d t
$$

for every solution $v$ of 21 ) with $v_{0} \in H_{0}^{1}(\Omega)$.

Proof of Lemma 2.0. Extend $v$ to $(-\pi, \pi)^{n} \times(0, T)$ in such a way that $v$ is an odd function of $x_{i}$ for each $i=1, \ldots, n$, and extend the initial state $v_{0}$ in a similar way. Then $v$ solves (10). Writing $v_{0}=\sum_{k \in \mathbb{Z}^{n}} c_{k} e^{i k \cdot x}$, we have that

$$
\nabla v(x, t)=\sum_{k \in \mathbb{Z}^{n}} i c_{k} e^{i\left(k \cdot x-|k|^{2} t\right)} k .
$$


It follows then from (12) that

$$
\begin{aligned}
\left\|\nabla v_{0}\right\|_{L^{2}\left(\mathbb{T}^{n}\right)}^{2} & =\sum_{j=1}^{n} \sum_{k \in \mathbb{Z}^{n}}\left|k_{j}\right|^{2}\left|c_{k}\right|^{2} \\
& \leq c \sum_{j=1}^{n} \int_{0}^{T} \int_{\omega}\left|\sum_{k \in \mathbb{Z}^{n}} c_{k} e^{i\left(k \cdot x-|k|^{2} t\right)} k_{j}\right|^{2} d x d t \\
& \leq c \int_{0}^{T} \int_{\omega}|\nabla v|^{2} d x d t
\end{aligned}
$$

The lemma is proved.

Step 2. We use the multiplier method to reduce the boundary observation inequality to an internal observation inequality. Without loss of generality, we may assume that $\Gamma_{0}$ is a (small) neighborhood of the vertex $M=(\pi, \ldots, \pi)$ defined as

$$
\Gamma_{0}=\left\{x \in \partial \Omega ; x_{1}+\cdots+x_{n}>n \pi-\varepsilon\right\},
$$

where $\varepsilon$ is a (possibly small) positive number. The following lemma is needed.

Lemma 2.7 There exists a nonnegative function $\theta \in C^{3}\left(\mathbb{R}^{n}\right)$ which is null on $\left\{x \in \mathbb{R}^{n} ; x_{1} \leq 0\right\}$ and strictly convex on $(0,+\infty)^{n} \cap B_{1}(0)$.

Proof of Lemma 2.7. Set $y^{+}=\max (y, 0)$ for all $y \in \mathbb{R}$. Let

$$
\theta\left(x_{1}, \ldots, x_{n}\right)=\left(x_{1}^{+}\right)^{4}\left(1+\delta \sum_{j=2}^{n}\left(x_{j}^{+}\right)^{4}\right)
$$

where $\delta>0$ is a small number whose value will be specified later. Clearly, $\theta$ is a nonnegative function of class $C^{3}$ on $\mathbb{R}^{n}$, which vanishes on the set $\left\{x_{1} \leq 0\right\}$. To prove that $\theta$ is strictly convex on $(0,+\infty)^{n} \cap B_{1}(0)$, it is sufficient to check that the Hessian matrix

$$
H(x)=\left(\frac{\partial^{2} \theta}{\partial x_{i} \partial x_{j}}(x)\right)
$$

is positive definite for every $x \in(0,+\infty)^{n} \cap B_{1}(0)$. Simple computations give that for any $\xi \in \mathbb{R}^{n}$,

$$
\xi^{T} H(x) \xi=12 x_{1}^{2}\left(1+\delta \sum_{j=2}^{n} x_{j}^{4}\right) \xi_{1}^{2}+12 \delta x_{1}^{4} \sum_{j=2}^{n} x_{j}^{2} \xi_{j}^{2}+32 \delta x_{1}^{3} \xi_{1} \sum_{j=2}^{n} x_{j}^{3} \xi_{j}
$$

From Young inequality, we obtain that

$$
32\left|x_{1}^{3} x_{j}^{3} \xi_{1} \xi_{j}\right| \leq 26 x_{1}^{2} x_{j}^{4} \xi_{1}^{2}+10 x_{1}^{4} x_{j}^{2} \xi_{j}^{2}
$$

therefore

$$
\xi^{T} H(x) \xi \geq(12-26(n-1) \delta) x_{1}^{2} \xi_{1}^{2}+2 \delta x_{1}^{4} \sum_{j=2}^{n} x_{j}^{2} \xi_{j}^{2} \geq c|\xi|^{2}
$$

if $x \in(0,+\infty)^{n} \cap B_{1}(0)$ and $\delta<(6 / 13)(n-1)^{-1}$.

At this position, we need an identity from 37 .

Lemma 2.8 [37, Lemma 2.2] For any $q \in H^{2}\left(\Omega, \mathbb{R}^{n}\right)$ and any solution $v$ of (21] issued from $v_{0} \in H_{0}^{1}(\Omega)$, it holds

$$
\begin{aligned}
& \frac{1}{2} \int_{0}^{T} \int_{\partial \Omega}(q \cdot \nu)\left|\frac{\partial v}{\partial \nu}\right|^{2} d \sigma d t=\left.\frac{1}{2} \operatorname{Im} \int_{\Omega}(v q \cdot \nabla \bar{v}) d x\right|_{0} ^{T} \\
& +\frac{1}{2} \operatorname{Re} \int_{0}^{T} \int_{\Omega}(v \nabla(\operatorname{div} q) \cdot \nabla \bar{v}) d x d t+\operatorname{Re} \int_{0}^{T} \int_{\Omega} \sum_{j, k=1}^{n} \frac{\partial q_{k}}{\partial x_{j}} \frac{\partial \bar{v}}{\partial x_{k}} \frac{\partial v}{\partial x_{j}} d x d t .
\end{aligned}
$$


Let

$$
\omega=\left\{x \in \Omega ; x_{1}+\cdots+x_{n}>n \pi-\varepsilon\right\} .
$$

We readily infer from Lemma 2.7 that there exists a convex function $\theta \in C^{3}(\bar{\Omega})$ which is strictly convex on $\omega$ and null on $\overline{\Omega \backslash \omega}$. Using (26) with $q=\nabla \theta$ we obtain

$$
\int_{0}^{T} \int_{\omega} \nabla \bar{v}(x)^{T} H(x) \nabla v(x) d x d t \leq c \int_{0}^{T} \int_{\Gamma_{0}}\left|\frac{\partial v}{\partial \nu}\right|^{2} d \sigma d t+C_{\delta} \int_{\Omega}\left|v_{0}\right|^{2} d x+\delta \int_{\Omega}\left|\nabla v_{0}\right|^{2} d x,
$$

where $\delta>0$ is a small number and $H(x)$ denotes the Hessian matrix given in (24). In (27), we used the fact that both quantities $\|v(t)\|_{L^{2}(\Omega)}$ and $\|\nabla v(t)\|_{L^{2}(\Omega)}$ are conserved. Using Lemma 2.6 and the fact that the Hessian matrix $H(x)=\left(\partial^{2} \theta / \partial x_{i} \partial x_{j}\right)(x)$ is positive definite on $\omega$, we obtain

$$
\left\|\nabla v_{0}\right\|_{L^{2}(\Omega)}^{2} \leq c \int_{0}^{T} \int_{\Gamma_{0}}\left|\frac{\partial v}{\partial \nu}\right|^{2} d \sigma d t+C_{\delta} \int_{\Omega}\left|v_{0}\right|^{2} d x .
$$

for a convenient choice of $\delta$. The proof of the estimate

$$
\left\|v_{0}\right\|_{L^{2}(\Omega)}^{2} \leq c \int_{0}^{T} \int_{\Gamma_{0}}\left|\frac{\partial v}{\partial \nu}\right|^{2} d \sigma d t
$$

is classical (see e.g. [37, pp. 27-28]). Then (22) follows from (28)-29). This completes the proof of Proposition 2.5 and of Theorem 2.4.

Remark 2.9 (i) Theorem 2.4 is stated for a square $\Omega=(0, \pi)^{n}$, but it is valid (with the same proof) for any rectangle $\Omega=\left(0, l_{1}\right) \times \cdots \times\left(0, l_{n}\right)$.

(ii) Using a frequential criterion and number theoretic arguments, Ramdani et al. 43 proved that when $n=2, \Gamma_{0} \subset \partial \Omega$ is a Dirichlet control domain if and only if $\Gamma_{0}$ has both a horizontal and a vertical components. It is however unclear whether the approach in [43] can yield a similar result for $n \geq 3$.

(iii) Using Theorem 2.9 on a rectangle $\tilde{\Omega}=(-1, \pi) \times(0, \pi)^{n-1}$ with a control input supported in $\tilde{\Omega} \backslash \Omega$, and next taking the restriction to $\Omega$, we infer that the linear Schrödinger equation is controllable in $L^{2}(\Omega)$ with a control supported on a side. (This fact can also be deduced from the Carleman inequalities established in [39].) This suggests that the condition for a domain to be a Dirichlet control domain is less restrictive when the state space is smoothed.

We now aim to extend Theorem 2.4 to a control result in a space $H^{s}(\Omega)$, with $s \geq-1$. We define $H_{D}^{s}(\Omega)=D\left(A_{D}^{\frac{s}{2}}\right)$, where $A_{D}$ is the Dirichlet Laplacian; i.e., $A_{D} u=-\Delta u$ with domain $D\left(A_{D}\right)=H^{2}(\Omega) \cap$ $H_{0}^{1}(\Omega) \subset L^{2}(\Omega)$. We first need to replace the characteristic function $1_{\Gamma_{0}}$ by a smooth controller function $g \in L^{\infty}(\partial \Omega)$. We adopt the following

Definition 2.10 Let $g \in L^{\infty}(\partial \Omega)$. We say that $g$ is a smooth Dirichlet controller if

(i) there exists a constant $C>0$ such that

$$
\left\|\nabla v_{0}\right\|_{L^{2}(\Omega)}^{2} \leq C \int_{0}^{T} \int_{\partial \Omega} g(x)\left|\frac{\partial v}{\partial \nu}\right|^{2} d \sigma d t
$$

for any solution $v$ of (21) emanating from $v_{0} \in H_{0}^{1}(\Omega)$ at $t=0$;

(i) for any face $F$ of $\partial \Omega, g_{F}=g_{\left.\right|_{F}} \in C^{\infty}(F)$ and for all $k \geq 0$

$$
\frac{\partial^{2 k+1} g_{F}}{\partial \nu^{2 k+1}}=0 \quad \text { on } \partial F \text {. }
$$

Note that for any nonempty open set $\Gamma_{0} \subset \partial \Omega$ one can construct a smooth Dirichlet controller $g$ supported in $\Gamma_{0}$. Consider for example a small neighborhood $\Gamma_{0}=[0, \varepsilon]^{n} \cap \partial \Omega$ of 0 in $\partial \Omega$. A smooth Dirichlet controller $g$ supported in $\Gamma_{0}$ is given by

$$
g\left(x_{1}, \ldots, x_{n}\right)=\prod_{i=1}^{n} \rho\left(x_{i}\right)
$$


where $\rho \in C^{\infty}(\mathbb{R})$ fulfills

$$
\rho(s)= \begin{cases}1 & \text { if } s \leq \frac{\varepsilon}{4} \\ 0 & \text { if } s \geq \frac{\varepsilon}{2}\end{cases}
$$

Note also that $g \in C^{0}(\partial \Omega)$ and that the set $\{x \in \partial \Omega ; g(x)>0\}$ is an open neighborhood of 0 in $\partial \Omega$.

Let $g$ be a smooth Dirichlet controller, and let $S$ denote the bounded operator $H_{0}^{1}(\Omega) \rightarrow H^{-1}(\Omega)$ defined by $S v_{0}=u(T)$, where $u=u(x, t)$ solves

$$
\begin{cases}i u_{t}+\Delta u=0 & \text { in } \Omega \times(0, T) \\ u=g(x) h(x, t) & \text { on } \partial \Omega \times(0, T) \\ u(0)=0 & \end{cases}
$$

with $h(x, t)=(\partial v / \partial \nu)(x, t), v=W_{D}(t) v_{0}$ denoting the solution of

$$
\begin{cases}i v_{t}+\Delta v=0 & \text { in } \Omega \times(0, T) \\ v=0 & \text { on } \partial \Omega \times(0, T) \\ v(0)=v_{0} & \end{cases}
$$

Applying HUM, we infer from the observability inequality (30) that $S$ is invertible. We shall prove that a similar result holds in more regular spaces.

Theorem 2.11 Pick any number $s \in\left[-1, \frac{1}{2}\right)$. Then $S$ is an isomorphism from $H_{D}^{s+2}(\Omega)$ onto $H_{D}^{s}(\Omega)$. More precisely, for any $T>0$ and any $u_{T} \in H_{D}^{s}(\Omega)$, if we set $h(x, t)=(\partial v / \partial \nu)(x, t)$ where $v$ denotes the solution of (33) with $v_{0}=S^{-1} u_{T}$, then $v_{0} \in H_{D}^{s+2}(\Omega), h \in H^{\frac{s+1}{2}}\left(0, T ; L^{2}(\partial \Omega)\right)$, and the solution $u$ of (32) satisfies $u \in C\left([0, T] ; H_{D}^{s}(\Omega)\right)$ and $u(T)=u_{T}$.

Proof. Step 1. Let us first check that $S^{-1}$ is a bounded operator from $H_{D}^{s}(\Omega)$ into $H_{D}^{s+2}(\Omega)$ for $s \in\left[-1, \frac{1}{2}\right)$. The result is already known for $s=-1$. Assume first that $-1<s<0$, and pick any $u_{T} \in H_{D}^{s}(\Omega)$ decomposed as

$$
u_{T}(x)=\sum_{p \in\left(\mathbb{N}^{*}\right)^{n}} u_{T, p} \sin \left(p_{1} x_{1}\right) \cdots \sin \left(p_{n} x_{n}\right),
$$

with $\sum_{p \in\left(\mathbb{N}^{*}\right)^{n}}|p|^{2 s}\left|u_{T, p}\right|^{2}<\infty$. Let $v_{0}=S^{-1}\left(u_{T}\right) \in H_{D}^{1}(\Omega)$ decomposed as

$$
v_{0}(x)=\sum_{p \in\left(\mathbb{N}^{*}\right)^{n}} v_{p} \sin \left(p_{1} x_{1}\right) \cdots \sin \left(p_{n} x_{n}\right),
$$

and let $v$ denote the solution of (33). The control given by HUM driving (32) from 0 to $u_{T}$ reads

$$
h(x, t):=\partial v / \partial \nu=\sum_{p \in\left(\mathbb{N}^{*}\right)^{n}} v_{p} e^{-i|p|^{2} t} \frac{\partial}{\partial \nu}\left(\sin \left(p_{1} x_{1}\right) \cdots \sin \left(p_{n} x_{n}\right)\right) .
$$

Let us write the solution $u=u(x, t)$ of $(32)$ in the form

$$
u(x, t)=\sum_{p \in\left(\mathbb{N}^{*}\right)^{n}} u_{p}(t) \sin \left(p_{1} x_{1}\right) \cdots \sin \left(p_{n} x_{n}\right) .
$$

The moments $\left\{u_{p}(t)\right\}_{p \in\left(\mathbb{N}^{*}\right)^{n}}$ can be computed from the control input $h$ by using duality. Scaling in (32) by $\bar{w}$, where $w=W_{D}(t) w_{0}$ is a smooth solution, we obtain

$$
i \int_{\Omega} u(x, t) \overline{w(x, t)} d x=\int_{0}^{t} \int_{\partial \Omega} g(x) h(x, \tilde{t}) \frac{\overline{\partial w}}{\partial \nu} d \sigma(x) d \tilde{t} .
$$

Pick any $q \in\left(\mathbb{N}^{*}\right)^{n}$ and choose $w_{0}(x)=\sin \left(q_{1} x_{1}\right) \cdots \sin \left(q_{n} x_{n}\right)$. We obtain from (35) that

$$
\begin{aligned}
\left(\frac{\pi}{2}\right)^{n} i e^{i|q|^{2} t} u_{q}(t)= & \int_{0}^{t} \int_{\partial \Omega} g(x) h(x, \tilde{t}) e^{i|q|^{2} \tilde{t}} \frac{\partial}{\partial \nu}\left(\sin \left(q_{1} x_{1}\right) \cdots \sin \left(q_{n} x_{n}\right)\right) d \sigma(x) d \tilde{t} \\
= & \sum_{p \in\left(\mathbb{N}^{*}\right)^{n}} v_{p}\left(\int_{0}^{t} e^{i\left(|q|^{2}-|p|^{2}\right) \tilde{t}} d \tilde{t}\right) \\
& \times \int_{\partial \Omega} g(x) \frac{\partial}{\partial \nu}\left(\sin \left(p_{1} x_{1}\right) \cdots \sin \left(p_{n} x_{n}\right)\right) \frac{\partial}{\partial \nu}\left(\sin \left(q_{1} x_{1}\right) \cdots \sin \left(q_{n} x_{n}\right)\right) d \sigma(x) .
\end{aligned}
$$


It follows that for $t=T$

$$
S\left(v_{0}\right)=u_{T}=u(T)=\sum_{q \in\left(\mathbb{N}^{*}\right)^{n}}\left(\sum_{p \in\left(\mathbb{N}^{*}\right)^{n}} a_{q, p} v_{p}\right) \sin \left(q_{1} x_{1}\right) \cdots \sin \left(q_{n} x_{n}\right)
$$

with

$$
a_{q, p}=-\left(\frac{2}{\pi}\right)^{n} \frac{e^{-i|p|^{2} T}-e^{-i|q|^{2} T}}{|q|^{2}-|p|^{2}} \int_{\partial \Omega} g(x) \frac{\partial}{\partial \nu}\left(\sin \left(p_{1} x_{1}\right) \cdots \sin \left(p_{n} x_{n}\right)\right) \frac{\partial}{\partial \nu}\left(\sin \left(q_{1} x_{1}\right) \cdots \sin \left(q_{n} x_{n}\right)\right) d \sigma(x) .
$$

In (39), we used the convention that

$$
\frac{e^{-i|p|^{2} t}-e^{-i|q|^{2} t}}{|q|^{2}-|p|^{2}}=i t e^{-i|q|^{2} t} \quad \text { for } \quad|p|=|q| .
$$

Introduce the operator $D^{\sigma}$ defined by

$$
D^{\sigma}\left(\sum_{p \in\left(\mathbb{N}^{*}\right)^{n}} c_{p} \sin \left(p_{1} x_{1}\right) \cdots \sin \left(p_{n} x_{n}\right)\right)=\sum_{p \in\left(\mathbb{N}^{*}\right)^{n}}|p|^{\sigma} c_{p} \sin \left(p_{1} x_{1}\right) \cdots \sin \left(p_{n} x_{n}\right)
$$

In what follows, $\sum_{p}$ and $\sum_{q}$ will stand for $\sum_{p \in\left(\mathbb{N}^{*}\right)^{n}}$ and $\sum_{q \in\left(\mathbb{N}^{*}\right)^{n}}$, respectively. We aim to prove that $v_{0} \in H_{D}^{s+2}(\Omega)$ for $u_{T} \in H_{D}^{s}(\Omega)$. For $v_{0}$ given by $(\underline{34})$, let

$$
\left\|v_{0}\right\|_{s}^{2}=\sum_{p}|p|^{2 s}\left|v_{p}\right|^{2}
$$

$C$ denoting a constant varying from line to line, we have that

$$
\begin{aligned}
\left\|v_{0}\right\|_{s+2} & \leq\left\|D^{s+1} v_{0}\right\|_{1} \\
& \leq C\left\|S\left(D^{s+1} v_{0}\right)\right\|_{-1} \\
& \leq C\left(\left\|D^{s+1}\left(S v_{0}\right)\right\|_{-1}+\left\|\left[S, D^{s+1}\right] v_{0}\right\|_{-1}\right) \\
& \leq C\left(\left\|u_{T}\right\|_{s}+\left\|\left[S, D^{s+1}\right] v_{0}\right\|_{-1}\right)
\end{aligned}
$$

Clearly

$$
\left[S, D^{s+1}\right] v_{0}=\sum_{q}\left(\sum_{p} a_{q, p}\left(|p|^{s+1}-|q|^{s+1}\right) v_{p}\right) \sin \left(q_{1} x_{1}\right) \cdots \sin \left(q_{n} x_{n}\right)
$$

hence

$$
\left\|\left[S, D^{s+1}\right] v_{0}\right\|_{-1}^{2}=\sum_{q}|q|^{-2}\left[\sum_{p} a_{q, p}\left(|p|^{s+1}-|q|^{s+1}\right) v_{p}\right]^{2} .
$$

Writing $\partial \Omega=\cup_{0 \leq l<2^{n}-1} F_{l}$, where the $F_{l}$ 's denote the faces of $\Omega$, the integral term in (39) may be written $\sum_{0 \leq l<2^{n}-1} I_{F_{l}}$, with

$$
I_{F_{l}}:=\int_{F_{l}} g(x) \frac{\partial}{\partial \nu}\left(\sin \left(p_{1} x_{1}\right) \cdots \sin \left(p_{n} x_{n}\right)\right) \frac{\partial}{\partial \nu}\left(\sin \left(q_{1} x_{1}\right) \cdots \sin \left(q_{n} x_{n}\right)\right) d \sigma(x) .
$$

Let us estimate $I_{F_{l}}$ for $F_{0}:=\left\{x \in \partial \Omega ; x_{n}=0\right\}=[0, \pi]^{n-1} \times\{0\}$. Then

$$
\begin{aligned}
\left|I_{F_{0}}\right| & =p_{n} q_{n}\left|\int_{[0, \pi]^{n-1}} g\left(x_{1}, \ldots, x_{n-1}, 0\right)\left[\prod_{j=1}^{n-1} \sin \left(p_{j} x_{j}\right) \sin \left(q_{j} x_{j}\right)\right] d x_{1} \cdots d x_{n-1}\right| \\
& =p_{n} q_{n}\left|\int_{[0, \pi]^{n-1}} g\left(x_{1}, \ldots, x_{n-1}, 0\right)\left[\prod_{j=1}^{n-1} \frac{1}{2}\left(\cos \left(p_{j}-q_{j}\right) x_{j}-\cos \left(p_{j}+q_{j}\right) x_{j}\right)\right] d x_{1} \cdots d x_{n-1}\right| .
\end{aligned}
$$

Using (31) and integrations by parts, we see that for every $k \in \mathbb{N}$, we have for some constant $C_{k}>0$

$$
\left|I_{F_{0}}\right| \leq C_{k} p_{n} q_{n} \prod_{j=1}^{n-1}\left\langle p_{j}-q_{j}\right\rangle^{-k}
$$


The corresponding contribution in $\left\|\left[S, D^{s+1}\right] v_{0}\right\|_{-1}^{2}$ is therefore estimated by

$$
A_{F_{0}}=\sum_{q}|q|^{-2}\left(\left.\sum_{p} p_{n} q_{n}\left(\prod_{j=1}^{n-1}\left\langle p_{j}-q_{j}\right\rangle^{-k}\right)\left\langle|q|^{2}-|p|^{2}\right\rangle^{-1}|| p\right|^{s+1}-|q|^{s+1}|| v_{p} \mid\right)^{2} .
$$

Since

$$
\frac{\left.|| p\right|^{s+1}-|q|^{s+1} \mid}{\left\langle|q|^{2}-|p|^{2}\right\rangle} \leq C \frac{|| p|-| q||\left(|p|^{s}+|q|^{s}\right)}{\left\langle|q|^{2}-|p|^{2}\right\rangle} \leq C \frac{|p|^{s}+|q|^{s}}{|p|+|q|}
$$

we have by Cauchy-Schwarz

$$
\begin{aligned}
A_{F_{0}} & \leq C \sum_{q}\left[\sum_{p} p_{n}\left(\prod_{j=1}^{n-1}\left\langle p_{j}-q_{j}\right\rangle^{-k}\right) \frac{|p|^{s}+|q|^{s}}{|p|+|q|}\left|v_{p}\right|\right]^{2} \\
& \leq C \sum_{q}\left(\sum_{p} \frac{|p|^{2 s}+|q|^{2 s}}{(|p|+|q|)^{2}} \prod_{j=1}^{n-1}\left\langle p_{j}-q_{j}\right\rangle^{-k}\right) \cdot\left(\sum_{p} p_{n}^{2}\left|v_{p}\right|^{2} \prod_{j=1}^{n-1}\left\langle p_{j}-q_{j}\right\rangle^{-k}\right)
\end{aligned}
$$

Pick any $k>1$. Then, as $s<0$, if we choose $k>1$

$$
\sum_{q_{n}} \sum_{p} \frac{|p|^{2 s}+|q|^{2 s}}{(|p|+|q|)^{2}} \prod_{j=1}^{n-1}\left\langle p_{j}-q_{j}\right\rangle^{-k} \leq \sum_{q_{n}} \sum_{p_{n}} \frac{p_{n}^{2 s}+q_{n}^{2 s}}{\left(p_{n}+q_{n}\right)^{2}} \sum_{p_{1}, \ldots, p_{n-1}} \prod_{j=1}^{n-1}\left\langle p_{j}-q_{j}\right\rangle^{-k}<\infty .
$$

Therefore

$$
\begin{aligned}
A_{F_{0}} & \leq C \sum_{q_{1}, \ldots, q_{n-1}} \sum_{p} p_{n}^{2}\left|v_{p}\right|^{2} \prod_{j=1}^{n-1}\left\langle p_{j}-q_{j}\right\rangle^{-k} \\
& \leq C \sum_{p}|p|^{2}\left|v_{p}\right|^{2} \sum_{q_{1}, \ldots, q_{n-1}} \prod_{j=1}^{n-1}\left\langle p_{j}-q_{j}\right\rangle^{-k} \\
& \leq C \sum_{p}|p|^{2}\left|v_{p}\right|^{2} .
\end{aligned}
$$

The estimate for another face $F_{l}$ is similar. We conclude that

$$
\left\|\left[S, D^{s+1}\right] v_{0}\right\|_{-1}^{2} \leq C\left\|v_{0}\right\|_{1}^{2} \leq C\left\|u_{T}\right\|_{-1}^{2}
$$

hence, with (41), $v_{0} \in H_{D}^{s+2}(\Omega)$. Let us now assume that $u_{T} \in H_{D}^{s}(\Omega)$ with $0 \leq s<\frac{1}{2}$. The proof is carried out as above when $-1<s<0$, except for the estimate of $A_{F_{0}}$ in (43). We know from the lines above that $v_{0} \in H_{D}^{\sigma}(\Omega)$ for any $\sigma<2$. Then, by Cauchy-Schwarz inequality,

$$
\begin{aligned}
A_{F_{0}} & \leq C \sum_{q}\left(\sum_{p} p_{n} \prod_{j=1}^{n-1}\left\langle p_{j}-q_{j}\right\rangle^{-k} \frac{|p|^{s}+|q|^{s}}{|p|+|q|}\left|v_{p}\right|\right)^{2} \\
& \leq C \sum_{q}\left(\sum_{p} \frac{|p|^{2 s}+|q|^{2 s}}{\left(|p|+|q|^{2}\right.}|p|^{-1} \prod_{j=1}^{n-1}\left\langle p_{j}-q_{j}\right\rangle^{-k}\right)\left(\sum_{p} p_{n}^{2}|p|\left|v_{p}\right|^{2} \prod_{j=1}^{n-1}\left\langle p_{j}-q_{j}\right\rangle^{-k}\right) .
\end{aligned}
$$

Note that

$$
\sum_{q_{n}}\left(\sum_{p} \frac{|p|^{2 s}+|q|^{2 s}}{(|p|+|q|)^{2}}|p|^{-1} \prod_{j=1}^{n-1}\left\langle p_{j}-q_{j}\right\rangle^{-k}\right) \leq C\left(S_{1}+S_{2}+S_{3}\right)
$$


where

$$
\begin{aligned}
& S_{1}=\sum_{q_{n}}\left(\sum_{p} \frac{|p|^{2 s-1}}{(|p|+|q|)^{2}} \prod_{j=1}^{n-1}\left\langle p_{j}-q_{j}\right\rangle^{-k}\right) \\
& S_{2}=\sum_{q_{n}}\left(\sum_{p} \frac{q_{n}^{2 s}|p|^{-1}}{(|p|+|q|)^{2}} \prod_{j=1}^{n-1}\left\langle p_{j}-q_{j}\right\rangle^{-k}\right) \\
& S_{3}=\sum_{q_{n}}\left(\sum_{p} \frac{\left|q^{\prime}\right|^{2 s}|p|^{-1}}{(|p|+|q|)^{2}} \prod_{j=1}^{n-1}\left\langle p_{j}-q_{j}\right\rangle^{-k}\right) \quad \text { where } q=\left(q^{\prime}, q_{n}\right) .
\end{aligned}
$$

Since $2 s-1<0$,

$$
S_{1} \leq \sum_{q_{n}}\left(\sum_{p} \frac{p_{n}^{2 s-1}}{\left(p_{n}+q_{n}\right)^{2}} \prod_{j=1}^{n-1}\left\langle p_{j}-q_{j}\right\rangle^{-k}\right) \leq \text { const }<\infty .
$$

Also,

$$
\begin{aligned}
S_{2} & \leq \sum_{q_{n}}\left(\sum_{p} \frac{q_{n}^{2 s} p_{n}^{-1}}{\left(p_{n}+q_{n}\right)^{2}} \prod_{j=1}^{n-1}\left\langle p_{j}-q_{j}\right\rangle^{-k}\right) \\
& \leq C \sum_{p_{n}} \sum_{q_{n}} \frac{q_{n}^{2 s} p_{n}^{-1}}{\left(p_{n}+q_{n}\right)^{2}} \\
& \leq C \sum_{p_{n}}\left(\frac{1}{p_{n}\left(p_{n}+1\right)}+p_{n}^{2 s-3}+\int_{1}^{\infty} \frac{x^{2 s}}{p_{n}\left(p_{n}+x\right)^{2}} d x\right) \\
& \leq C\left(1+\sum_{p_{n} \geq 1} p_{n}^{2 s-2} \int_{0}^{+\infty} \frac{y^{2 s}}{(1+y)^{2}} d y\right) \\
& \leq \text { const }<\infty .
\end{aligned}
$$

Finally,

$$
S_{3} \leq\left|q^{\prime}\right|^{2 s} \sum_{q_{n}} \sum_{p} \frac{p_{n}^{-1}}{\left(p_{n}+q_{n}\right)^{2}} \prod_{j=1}^{n-1}\left\langle p_{j}-q_{j}\right\rangle^{-k} \leq C\left|q^{\prime}\right|^{2 s}
$$

It follows that

$$
A_{F_{0}} \leq C \sum_{q_{1}, \ldots, q_{n-1}} \sum_{p} p_{n}^{2}|p|\left|v_{p}\right|^{2}\left|q^{\prime}\right|^{2 s} \prod_{j=1}^{n-1}\left\langle p_{j}-q_{j}\right\rangle^{-k} .
$$

Note that

$$
\sum_{q_{1}, \ldots, q_{n-1}}\left|q^{\prime}\right|^{2 s} \prod_{j=1}^{n-1}\left\langle p_{j}-q_{j}\right\rangle^{-k} \leq C\left|p^{\prime}\right|^{2 s}
$$

since, for $k>2 s+1$,

$$
\sum_{q_{j}} q_{j}^{2 s}\left\langle p_{j}-q_{j}\right\rangle^{-k} \leq C p_{j}^{2 s}
$$

(Split the sum into one for $q_{j} \leq 2 p_{j}$, and another one for $q_{j}>2 p_{j}$.) Therefore, since $0 \leq s<1 / 2$,

$$
A_{F_{0}} \leq C \sum_{p}|p|^{3+2 s}\left|v_{p}\right|^{2}=\left\|v_{0}\right\|_{s+\frac{3}{2}}^{2} \leq C|| u_{T} \|_{s-\frac{1}{2}}^{2} .
$$

Thus, we have proved that $S^{-1}$ is bounded from $H_{D}^{s}(\Omega)$ into $H_{D}^{s+2}(\Omega)$ for $-1 \leq s<\frac{1}{2}$. Note that, for $v_{0} \in H_{D}^{s+2}(\Omega), h \in H^{\frac{s+1}{2}}\left(\mathbb{T} ; L^{2}(\partial \Omega)\right)$ by (35).

Step 2. Since $S$ is an isomorphism from $H_{D}^{1}(\Omega)$ onto $H_{D}^{-1}(\Omega)$, it remains to prove that $S$ maps $H_{D}^{s+2}(\Omega)$ into $H_{D}^{s}(\Omega)$. The proof of Theorem 2.11 will thus be complete with the following result. 
Proposition 2.12 Let $s \in\left[-1, \frac{1}{2}\right)$ and $T>0$. For any $v_{0} \in H_{D}^{s+2}(\Omega)$, let $u=\Gamma v_{0}$ denote the solution of (32) associated with $h=\partial v / \partial \nu$, where $v(t)=W_{D}(t) v_{0}$. Then $\Gamma$ is a bounded operator from $H_{D}^{s+2}(\Omega)$ into $C\left([0, T] ; H_{D}^{s}(\Omega)\right)$.

Proof of Proposition 2.19. It is well known that for any $h \in L^{2}\left(0, T ; L^{2}(\partial \Omega)\right)$, there exists a unique solution $u \in C\left([0, T] ; H^{-1}(\Omega)\right)$ in the transposition sense of (32) (see e.g. [37). The result is therefore true for $s=-1$. Let us now assume that $s \in(-1,1 / 2)$. From Step 1 , we know that $u$ is given by

$$
u(t)=-\left(\frac{2}{\pi}\right)^{n} \sum_{q \in\left(\mathbb{N}^{*}\right)^{n}}\left(\sum_{p \in\left(\mathbb{N}^{*}\right)^{n}} v_{p} \frac{e^{-i|p|^{2} t}-e^{-i|q|^{2} t}}{|q|^{2}-|p|^{2}} I(g, p, q)\right) \sin \left(q_{1} x_{1}\right) \cdots \sin \left(q_{n} x_{n}\right)
$$

where

$$
I(g, p, q)=\int_{\partial \Omega} g(x) \frac{\partial}{\partial \nu}\left(\sin \left(p_{1} x_{1}\right) \cdots \sin \left(p_{n} x_{n}\right)\right) \frac{\partial}{\partial \nu}\left(\sin \left(q_{1} x_{1}\right) \cdots \sin \left(q_{n} x_{n}\right)\right) d \sigma(x) .
$$

Again $I(g, p, q)=\sum_{0 \leq l<2^{n}-1} I_{F_{l}}$, where the $F_{l}$ 's denote the faces of $\Omega$ and $I_{F_{l}}$ is given in (37). We have that

$$
\begin{aligned}
\left\|\Gamma v_{0}\right\|_{L^{\infty}\left(0, T ; H_{D}^{s}(\Omega)\right)} & =\left\|D^{s+1}\left(\Gamma v_{0}\right)\right\|_{L^{\infty}\left(0, T ; H_{D}^{-1}(\Omega)\right)} \\
& \leq\left\|\Gamma\left(D^{s+1} v_{0}\right)\right\|_{L^{\infty}\left(0, T ; H_{D}^{-1}(\Omega)\right)}+\left\|\left[\Gamma, D^{s+1}\right] v_{0}\right\|_{L^{\infty}\left(0, T ; H_{D}^{-1}(\Omega)\right)}
\end{aligned}
$$

Since

$$
\left\|\Gamma\left(D^{s+1} v_{0}\right)\right\|_{L^{\infty}\left(0, T ; H_{D}^{-1}(\Omega)\right)} \leq C\left\|D^{s+1} v_{0}\right\|_{1} \leq C\left\|v_{0}\right\|_{s+2},
$$

it remains to estimate the commutator $\left[\Gamma, D^{s+1}\right] v_{0}$. Clearly

$$
\left(\left[\Gamma, D^{s+1}\right] v_{0}\right)(t)=-\left(\frac{2}{\pi}\right)^{n} \sum_{q}\left(\sum_{p ;|p| \neq|q|} v_{p} \frac{|p|^{s+1}-|q|^{s+1}}{|q|^{2}-|p|^{2}}\left(e^{-i|p|^{2} t}-e^{-i|q|^{2} t}\right) I(g, p, q)\right) \prod_{j=1}^{n} \sin \left(q_{j} x_{j}\right) .
$$

The contribution in $\left\|\left(\left[\Gamma, D^{s+1}\right] v_{0}\right)(t)\right\|_{-1}^{2}$ due to $F_{0}=\left\{x \in \partial \Omega ; x_{n}=0\right\}$ is estimated with (42) by

$$
\begin{aligned}
B_{F_{0}} & \leq C \sum_{q}|q|^{-2}\left(\sum_{p,|p| \neq|q|}\left|v_{p}\right| \frac{|p|^{s}+|q|^{s}}{|p|+|q|}\left|I_{F_{0}}\right|\right)^{2} \\
& \leq C \sum_{q}\left(\sum_{p ;|p| \neq|q|}\left|v_{p}\right| \frac{|p|^{s}+|q|^{s}}{|p|+|q|^{2}} p_{n} \prod_{j=1}^{n-1}\left\langle p_{j}-q_{j}\right\rangle^{-k}\right)^{2} .
\end{aligned}
$$

Therefore, using the estimation of the r.h.s. of (44) in (45), we conclude that for $s<1 / 2$

$$
B_{F_{0}} \leq C\left\|v_{0}\right\|_{s+\frac{3}{2}}^{2}
$$

the constant $C$ being uniform in $t \in[0, T]$. Therefore

$$
\left\|\left[\Gamma, D^{s+1}\right] v_{0}\right\|_{L^{\infty}\left(0, T ; H_{D}^{-1}(\Omega)\right)} \leq C\left\|v_{0}\right\|_{s+2} .
$$

Thus, we have proved that

$$
\|u\|_{L^{\infty}\left(0, T ; H_{D}^{s}(\Omega)\right)} \leq C\left\|v_{0}\right\|_{H_{D}^{s+2}(\Omega)} .
$$

Since $u \in C\left([0, T] ; H_{D}^{-1}(\Omega)\right)$, we conclude that $u \in C_{w}\left([0, T] ; H_{D}^{s}(\Omega)\right)$. If we pick $\tilde{s} \in(s, 1 / 2)$ and $\tilde{v}_{0} \in H_{D}^{\tilde{s}+2}(\Omega)$, the corresponding solution $\tilde{u}$ belongs to $C_{w}\left([0, T] ; H_{D}^{\tilde{s}}(\Omega)\right)$, hence to $C\left([0, T] ; H_{D}^{s}(\Omega)\right)$, the embedding $H_{D}^{\tilde{s}}(\Omega) \subset H_{D}^{s}(\Omega)$ being compact. It follows from $(49)$ combined to the density of $H_{D}^{\tilde{s}+2}(\Omega)$ in $H_{D}^{s+2}(\Omega)$ that $u \in C\left([0, T] ; H_{D}^{s}(\Omega)\right)$ for $v_{0} \in H_{D}^{s+2}(\Omega)$. In particular, $u(T) \in H_{D}^{s}(\Omega)$, so that $S$ is an isomorphism from $H_{D}^{s+2}(\Omega)$ onto $H_{D}^{s}(\Omega)$. This completes the proof of Proposition 2.12 and of Theorem 2.11. 


\subsubsection{Neumann boundary control}

We adopt the following definition.

Definition 2.13 The open set $\Gamma_{0} \subset \partial \Omega$ is called a Neumann control domain if given any $u_{0}, u_{1} \in L^{2}(\Omega)$ and any time $T>0$, one may find a control $h \in L^{2}\left(0, T ; L^{2}\left(\Gamma_{0}\right)\right)$ such that the solution $u=u(x, t)$ of

$$
\begin{cases}i u_{t}+\Delta u=0 & \text { in } \Omega \times(0, T) \\ \frac{\partial u}{\partial \nu}=1_{\Gamma_{0}} h(x, t) & \text { on } \partial \Omega \times(0, T) \\ u(0)=u_{0} & \end{cases}
$$

satisfies $u(T)=u_{1}$.

The following result provides Neumann control domains in any dimension $n \geq 2$.

Proposition 2.14 Let $\Omega=(0, \pi)^{n}$, and let $\Gamma_{0} \subset \partial \Omega$ be a side of $\Omega$. Then $\Gamma_{0}$ is a Neumann control domain.

Proof. Assume e.g. that $\Gamma_{0}=\{0\} \times(0, \pi)^{n-1}$. By Dolecki-Russell criterion, we only have to check the following observability inequality

$$
\left\|v_{0}\right\|_{L^{2}(\Omega)}^{2} \leq C \int_{0}^{T} \int_{\Gamma_{0}}|v(x, t)|^{2} d \sigma d t
$$

where $v_{0}$ is any function in $L^{2}(\Omega)$ and $v=v(x, t)$ solves

$$
\begin{cases}i v_{t}+\Delta v=0 & \text { in } \Omega \times(0, T) \\ \frac{\partial v}{\partial \nu}=0 & \text { on } \partial \Omega \times(0, T) \\ v(0)=v_{0} . & \end{cases}
$$

Expanding $v_{0}$ as

$$
v_{0}(x)=\sum_{k \in \mathbb{N}^{n}} c_{k} \cos \left(k_{1} x_{1}\right) \cdots \cos \left(k_{n} x_{n}\right),
$$

then the corresponding solution $v(x, t)$ reads

$$
v(x, t)=\sum_{k \in \mathbb{N}^{n}} c_{k} e^{-i|k|^{2} t} \cos \left(k_{1} x_{1}\right) \cdots \cos \left(k_{n} x_{n}\right) .
$$

It follows that

$$
\begin{aligned}
\int_{0}^{T} \int_{\Gamma_{0}}|v(x, t)|^{2} d \sigma d t & =\int_{0}^{T} \int_{(0, \pi)^{n-1}}\left|\sum_{k \in \mathbb{N}^{n}} c_{k} e^{-i|k|^{2} t} \cos \left(k_{2} x_{2}\right) \cdots \cos \left(k_{n} x_{n}\right)\right|^{2} d x_{2} \cdots d x_{n} d t \\
& \sim \sum_{k_{2}, \ldots, k_{n} \geq 0} \int_{0}^{T}\left|\sum_{k_{1} \geq 0} c_{k} e^{-i k_{1}^{2} t}\right|^{2} d t \sim \sum_{k \in \mathbb{N}^{n}}\left|c_{k}\right|^{2} \sim\left\|v_{0}\right\|_{L^{2}(\Omega)}^{2},
\end{aligned}
$$

where we used the orthogonality of the functions $\cos \left(k_{2} x_{2}\right) \cdots \cos \left(k_{n} x_{n}\right)$ in $L^{2}\left(\Gamma_{0}\right)$ and Ingham's lemma.

We now aim to extend Proposition 2.14 to a control result in a space $H^{s}(\Omega), s>0$. We define $H_{N}^{s}(\Omega)=$ $D\left(A_{N}^{\frac{s}{2}}\right)$, where $A_{N}$ is the Neumann Laplacian (i.e. $A_{N} u=u-\Delta u$ with $D\left(A_{N}\right)=\left\{u \in H^{2}(\Omega), \partial u / \partial \nu=\right.$ 0 on $\left.\partial \Omega\} \subset L^{2}(\Omega)\right)$. A result similar to Theorem 2.11 may be obtained along the same lines. We limit ourselves to giving a weaker result with a very short proof.

Theorem 2.15 Let $\Gamma_{0}$ be a Neumann control domain, $T=2 \pi, s \in[0,1)$ and $u_{0}, u_{1} \in H_{N}^{s}(\Omega)$. Then there exists a control input $h \in H^{\frac{s}{2}}\left(\mathbb{T} ; L^{2}(\partial \Omega)\right)$ such that the solution $u$ of (50) satisfies $u(T)=u_{1}$.

Proof. Without loss of generality, we may assume that $u_{0}=0$. A direct computation shows that for any (smooth) solution $u$ of (50) emanating from $u_{0}=0$ and any (smooth) solution $v$ of (52), it holds

$$
i \int_{\Omega} u(x, T) \overline{v(x, T)} d x=-\int_{0}^{T} \int_{\partial \Omega} 1_{\Gamma_{0}} h(x, t) \bar{v} d \sigma d t .
$$


As usual, for any $h \in L^{2}\left(0, T ; L^{2}(\partial \Omega)\right)$, the solution $u \in C\left([0, T] ; L^{2}(\Omega)\right)$ of $(50)$ is defined by

$$
i(u(t), v(t))_{L^{2}(\Omega)}=-\left(h, 1_{\Gamma_{0}} v\right)_{L^{2}\left(0, t ; L^{2}(\partial \Omega)\right)}, \quad \forall t \in[0, T], \forall v_{0} \in L^{2}(\Omega)
$$

where $v(t)$ solves (52).

Claim 1. If $v_{0} \in H_{N}^{-s}(\Omega)$ for some $s \in \mathbb{R}$, then $v \in H^{-\frac{s}{2}}\left(\mathbb{T} ; L^{2}(\partial \Omega)\right)$.

Indeed, if we write $v_{0}=\sum_{k \in \mathbb{N}^{n}} c_{k} \cos \left(k_{1} x_{1}\right) \cdots \cos \left(k_{n} x_{n}\right)$ and

$$
v(x, t)=\sum_{k \in \mathbb{N}^{n}} c_{k} e^{-i|k|^{2} t} \cos \left(k_{1} x_{1}\right) \cdots \cos \left(k_{n} x_{n}\right)
$$

then we have that

$$
\|v\|_{H^{-\frac{s}{2}}\left(\mathbb{T}, L^{2}(\partial \Omega)\right)}^{2} \sim \sum_{k}\left(1+|k|^{2}\right)^{-s}\left|c_{k}\right|^{2} \sim\left\|v_{0}\right\|_{H_{N}^{-s}(\Omega)}^{2}
$$

We may rewrite 53 in the form

$$
i\langle u(T), v(T)\rangle_{H_{N}^{s}, H_{N}^{-s}}=-\left\langle h, 1_{\Gamma_{0}} v\right\rangle_{H^{\frac{s}{2}}\left(\mathbb{T} ; L^{2}(\partial \Omega)\right), H^{-\frac{s}{2}}\left(\mathbb{T} ; L^{2}(\partial \Omega)\right)} \cdot
$$

Note that $u \in C\left([0, T] ; H_{N}^{s}(\Omega)\right)$ if $0 \leq s<1$. It remains to establish the following

Claim 2. (Observability inequality) The following estimate holds for the solutions of (52):

$$
\left\|1_{\Gamma_{0}} v\right\|_{H^{-\frac{s}{2}}\left(\mathbb{T} ; L^{2}(\partial \Omega)\right)}^{2} \geq \mathrm{const}\left\|v_{0}\right\|_{H_{N}^{-s}(\Omega)}^{2}
$$

If (57) is not true, one can construct a sequence $\left\{v_{j}\right\}$ such that

$$
j\left\|1_{\Gamma_{0}} v_{j}\right\|_{H^{-\frac{s}{2}}\left(\mathbb{T} ; L^{2}(\partial \Omega)\right)}^{2}<\left\|v_{j}(0)\right\|_{H_{N}^{-s}(\Omega)}^{2}=1 .
$$

Let $w_{j}=\left(1-\partial_{t}^{2}\right)_{p}^{-\frac{s}{4}} v_{j}$, where for any $\sigma \in \mathbb{R}$

$$
\left(1-\partial_{t}^{2}\right)_{p}^{\sigma} \sum_{l \in \mathbb{Z}} c_{l} e^{i l t}=\sum_{l \in \mathbb{Z}}\left(1+|l|^{2}\right)^{\sigma} c_{l} e^{i l t} .
$$

Then $w_{j}$ solves (52) with $w_{j}(0)$ substituted to $v_{0}$, and from (58) we obtain

$$
1_{\Gamma_{0}} w_{j} \rightarrow 0 \text { in } L^{2}\left(\mathbb{T} ; L^{2}(\partial \Omega)\right) .
$$

As $\Gamma_{0}$ is a Neumann control domain, we infer that $w_{j}(0) \rightarrow 0$ in $L^{2}(\Omega)$, hence

$$
w_{j} \rightarrow 0 \text { in } L^{2}\left(\mathbb{T} ; L^{2}(\partial \Omega)\right) .
$$

This gives

$$
v_{j} \rightarrow 0 \text { in } H^{-\frac{s}{2}}\left(\mathbb{T} ; L^{2}(\partial \Omega)\right) .
$$

Using (55), we infer that $v_{j}(0) \rightarrow 0$ in $H_{N}^{-s}(\Omega)$, which contradicts (58). This completes the proof of Theorem 2.15 .

\section{Nonlinear systems}

\subsection{Internal control}

In this section we consider the following nonlinear control system

$$
\left\{\begin{array}{l}
i u_{t}+\Delta u+N(u)=i G h=i a(x) h(x, t), x \in \mathbb{T}^{n}, t>0, \\
u(x, 0)=\phi(x),
\end{array}\right.
$$

where $a \in C^{\infty}\left(\mathbb{T}^{n}\right)$, and the nonlinearity $N(u)$ reads

$$
N(u)=\lambda u^{\alpha_{1}} \bar{u}^{\alpha_{2}}, \quad \alpha_{1}+\alpha_{2}=: \alpha+1 \geq 2,
$$


with $\lambda \in \mathbb{R}$, and $\alpha, \alpha_{1}, \alpha_{2} \in \mathbb{N}$. Note that for any $\alpha=2 \beta \in 2 \mathbb{N}^{*},|u|^{\alpha} u=u^{\beta+1} \bar{u}^{\beta}$.

We introduce the number

$$
s_{\alpha, n}= \begin{cases}\frac{n}{2}-1 & \text { if } \alpha=1, \\ \frac{n}{2}-\frac{3}{4}-\frac{1}{4(n-1)} & \text { if } \alpha=2, \\ \frac{n}{2}-\frac{2}{\alpha} & \text { if } \alpha \geq 3 .\end{cases}
$$

Thus $s_{\alpha, n}=s_{c}:=\frac{n}{2}-\frac{2}{\alpha}$ (the critical Sobolev exponent obtained by scaling in NLS) for $\alpha \geq 3$, while $s_{\alpha, n}>s_{c}$ for $\alpha=1,2$ (except for $n=\alpha=2$ where $s_{2,2}=s_{c}=0$ ).

By Corollary 3.5 (see below), the system (60) is locally well-posed in the space $H^{s}\left(\mathbb{T}^{n}\right)$ for $\alpha \geq 1$ and $s>s_{\alpha, n}$ with $\phi \in H^{s}\left(\mathbb{T}^{n}\right)$ and $h \in L_{l o c}^{2}\left(\mathbb{R}, H^{s}\left(\mathbb{T}^{n}\right)\right)$.

Our main concern is its exact controllability in the space $H^{s}\left(\mathbb{T}^{n}\right)$.

Theorem 3.1 For given $n \geq 2, \alpha_{1}, \alpha_{2} \in \mathbb{N}$ with $\alpha_{1}+\alpha_{2}=: \alpha+1 \geq 2$, and $a \not \equiv 0$, the system (60) is locally exactly controllable in the space $H^{s}\left(\mathbb{T}^{n}\right)$ for any $s>s_{\alpha, n}$. More precisely, for any given $T>0$, there exists a number $\delta>0$ depending on $\alpha, n, T$ and $\lambda$ such that if $\phi, \psi \in H^{s}\left(\mathbb{T}^{n}\right)$ satisfy

$$
\|\phi\|_{s} \leq \delta, \quad\|\psi\|_{s} \leq \delta
$$

then one can choose a control input $h \in L^{2}\left(0, T ; H^{s}\left(\mathbb{T}^{n}\right)\right)$ such that the system (60) admits a solution $u \in C\left([0, T] ; H^{s}\left(\mathbb{T}^{n}\right)\right)$ satisfying

$$
u(x, 0)=\phi(x), \quad u(x, T)=\psi(x) .
$$

The system (60) can be rewritten in its equivalent integral form

$$
u(t)=W(t) \phi+i \int_{0}^{t} W(t-\tau)(N(u)(\tau)) d \tau+\int_{0}^{t} W(t-\tau)[G h](\tau) d \tau .
$$

To prove Theorem 3.1, a smoothing property is needed for the operator from $f$ to $u$, where

$$
u(t)=\int_{0}^{t} W(t-\tau) f(\tau) d \tau .
$$

This needed smoothing property was provided in Bourgain's work [8, 9] where he dealt with the Cauchy problem for the periodic Schrödinger equation.

For given $s, b \in \mathbb{R}$, the Bourgain space $X_{s, b}$ is the space of functions $u: \mathbb{T}^{n} \times \mathbb{R} \rightarrow \mathbb{C}$ for which the norm

$$
\|u\|_{X_{s, b}}=\|W(-t) u(., t)\|_{H_{t}^{b}\left(H_{x}^{s}\right)}
$$

is finite. Decomposing $u$ as

$$
u(x, t)=\sum_{k \in \mathbb{Z}^{n}} \int_{\mathbb{R}} \hat{u}(k, \tau) e^{i(k \cdot x+\tau t)} d \tau
$$

we have that

$$
\|u\|_{X_{s, b}}^{2}=\sum_{k \in \mathbb{Z}^{n}} \int_{\mathbb{R}}\left\langle\tau+|k|^{2}\right\rangle^{2 b}\langle k\rangle^{2 s}|\hat{u}(k, \tau)|^{2} d \tau
$$

where $\langle y\rangle:=\left(1+|y|^{2}\right)^{\frac{1}{2}}$. For given $T>0, X_{s, b}^{T}$ is the restriction norm space

$$
X_{s, b}^{T}=\left\{u_{\mid \mathbb{T}^{n} \times(0, T)} ; u \in X_{s, b}\right\}
$$

with the restriction norm

$$
\|u\|_{X_{s, b}^{T}}=\inf \left\{\|\tilde{u}\|_{X_{s, b}} ; \tilde{u} \in X_{s, b}, \tilde{u}_{\mid \mathbb{T}^{n} \times(0, T)}=u\right\} .
$$

Before we proceed to show the exact controllability results, we present the two following technical lemmas (see e.g. [50]) which play important roles in the proof of Theorem 3.1 .

Lemma 3.2 For given $T>0$ and $s, b \in \mathbb{R}$, there exists a constant $C>0$ such that

$$
\|W(t) \phi\|_{X_{s, b}^{T}} \leq C\|\phi\|_{s}
$$

for any $\phi \in H^{s}\left(\mathbb{T}^{n}\right)$. 
Lemma 3.3 For given $T>0, b>1 / 2$, and $s \in \mathbb{R}$, there exists a constant $C>0$ such that

$$
\left\|\int_{0}^{t} W(t-\tau) f(\tau) d \tau\right\|_{X_{s, b}^{T}} \leq C\|f\|_{X_{s, b-1}^{T}}
$$

for any $f \in X_{s, b-1}^{T}$.

The following multilinear estimate is crucial when applying the contraction mapping theorem.

Proposition 3.4 Let $n \geq 2, \alpha \in \mathbb{N}^{*}$ and $s>s_{\alpha, n}$. Then there exist some numbers $b \in\left(0, \frac{1}{2}\right)$ and $C>0$ such that

$$
\left\|\prod_{i=1}^{\alpha+1} \tilde{u}_{i}\right\|_{X_{s,-b}} \leq C \prod_{i=1}^{\alpha+1}\left\|u_{i}\right\|_{X_{s, b}} \quad \forall u_{1}, \ldots, u_{\alpha+1} \in X_{s, b}
$$

where $\tilde{u}_{i}$ denotes $u_{i}$ or $\overline{u_{i}}$.

Corollary 3.5 Let $n \geq 2, \alpha \in \mathbb{N}^{*}$, and $s>s_{\alpha, n}$. Pick $u_{0} \in H^{s}\left(\mathbb{T}^{n}\right)$ and $h \in X_{s, 0}=L^{2}\left(\mathbb{R} ; H^{s}\left(\mathbb{T}^{n}\right)\right)$. Then there exist two numbers $b>\frac{1}{2}$ and $T=T\left(\left\|u_{0}\right\|_{H^{s}\left(\mathbb{T}^{n}\right)},\|h\|_{X_{s, 0}}\right)$ so that the initial-value problem (60) admits a unique solution $u \in X_{s, b}^{T}$.

Remark 3.6 Proposition 3.4, which is proved in Appendix for the sake of completeness, is essentially due to Bourgain. It was proved in [9] when $\alpha=n=2$, and in [B] in Besov-type spaces when $s>s_{b}$, where

$$
s_{b}= \begin{cases}s_{c} & \text { if } n=2 \\ \max \left(s_{c}, \frac{3}{4}\right) & \text { if } n=3 \\ \max \left(s_{c}, \frac{3 n}{n+4}\right) & \text { if } n \geq 4\end{cases}
$$

Notice that $s_{b}>s_{c}$ only for $(\alpha, n) \in\{(2,3),(2,4),(2,5),(3,4)\}$. The corresponding values of $s_{b}, s_{c}$ and $s_{\alpha, n}$ are reported in Table 1. On the other hand, $s_{b}=s_{c}<s_{\alpha, n}$ for $\alpha=2$ and $n \geq 6$. Sharp results for the

\begin{tabular}{|l|c|c|c|c|}
\hline$(\alpha, n)$ & $(2,3)$ & $(2,4)$ & $(2,5)$ & $(3,4)$ \\
\hline$s_{b}$ & $\frac{3}{4}$ & $\frac{3}{2}$ & $\frac{5}{3}$ & $\frac{3}{2}$ \\
\hline$s_{\alpha, n}$ & $\frac{5}{8}$ & $\frac{7}{6}$ & $\frac{27}{16}$ & $\frac{4}{3}$ \\
\hline$s_{c}$ & $\frac{1}{2}$ & 1 & $\frac{3}{2}$ & $\frac{4}{3}$ \\
\hline
\end{tabular}

Table 1: $s_{b}, s_{\alpha, n}$ and $s_{c}$ for $(\alpha, n) \in\{(2,3),(2,4),(2,5),(3,4)\}$

local well-posedness of $N L S$ on $\mathbb{T}^{n}$ are also given in [2] for $\alpha=n=1$, and in [17] for $\left(\alpha_{1}, \alpha_{2}\right)=(0,2)$ and $2 \leq n \leq 4$.

It follows at once from Proposition 3.4 that for any $T>0, s>s_{\alpha, n}$, and some $b>1 / 2, b^{\prime}>b-1$ we have

$$
\|N(v)-N(w)\|_{X_{s, b^{\prime}}^{T}} \leq C\left(\|v\|_{X_{s, b}^{T}}^{\alpha}+\|w\|_{X_{s, b}^{T}}^{\alpha}\right)\|v-w\|_{X_{s, b}^{T}} \quad \forall v, w \in X_{s, b}^{T} .
$$

We are now in a position to give a proof of Theorem 3.1.

Proof of Theorem 3.1: Set

$$
\omega(v, T)=i \int_{0}^{T} W(T-\tau) N(v)(\tau) d \tau
$$

By Theorem 2.2, if we choose

$$
h=\Phi(\phi, \psi-\omega(v, T)),
$$


then

$$
\begin{gathered}
W(t) \phi+\int_{0}^{t} W(t-\tau)(i N(v)+G \Phi(\phi, \psi-\omega(v, T))(\tau) d \tau \\
= \begin{cases}\phi(x) \text { in } \mathbb{T}^{n} & \text { when } t=0 ; \\
\psi(x)-\omega(v, T)+\omega(v, T)=\psi(x) \text { in } \mathbb{T}^{n}, & \text { when } t=T .\end{cases}
\end{gathered}
$$

It suggests us to consider the nonlinear map:

$$
\Gamma(v)=W(t) \phi+i \int_{0}^{t} W(t-\tau)(i N(v)+G \Phi(\phi, \psi-\omega(v, T))(\tau) d \tau .
$$

The proof would be complete if we can show that this map $\Gamma$ has a fixed point in the space $X_{s, b}^{T}$, with $b \in\left(\frac{1}{2}, 1\right)$.

To this end, note that by using Lemma 3.2, Lemma 3.3 and Proposition 3.4, there exist a number $b \in\left(\frac{1}{2}, 1\right)$ and some constants $C_{j}, j=1,2,3$ such that

$$
\|\Gamma(v)\|_{X_{s, b}^{T}} \leq C_{1}\left(\|\phi\|_{s}+\|\psi\|_{s}+\|\omega(v, T)\|_{s}\right)+C_{2}\|v\|_{X_{s, b}^{T}}^{\alpha+1}
$$

for any $v \in X_{s, b}^{T}$ and

$$
\left\|\Gamma\left(v_{1}\right)-\Gamma\left(v_{2}\right)\right\|_{X_{s, b}^{T}} \leq C_{1}\left\|\omega\left(v_{1}, T\right)-\omega\left(v_{2}, T\right)\right\|_{s}+C_{3}\left(\left\|v_{1}\right\|_{X_{s, b}^{T}}^{\alpha}+\left\|v_{2}\right\|_{X_{s, b}^{T}}^{\alpha}\right)\left\|v_{1}-v_{2}\right\|_{X_{s, b}^{T}}
$$

for any $v_{1}, v_{2} \in X_{s, b}^{T}$. Note that there exists a constant $C_{4}>0$ such that

$$
\begin{aligned}
\|\omega(v, T)\|_{s} & \leq\left\|\int_{0}^{t} W(t-\tau) N(v)(\tau) d \tau\right\|_{C\left([0, T] ; H^{s}\left(\mathbb{T}^{n}\right)\right)} \\
& \leq \mathrm{const}\left\|\int_{0}^{t} W(t-\tau) N(v)(\tau) d \tau\right\|_{X_{s, b}^{T}} \\
& \leq C_{4}\|v\|_{X_{s, b}^{T}}^{\alpha+1} .
\end{aligned}
$$

Similarly

$$
\left\|\omega\left(v_{1}, T\right)-\omega\left(v_{2}, T\right)\right\|_{s} \leq C_{5}\left(\left\|v_{1}\right\|_{X_{s, b}^{T}}^{\alpha}+\left\|v_{2}\right\|_{X_{s, b}^{T}}^{\alpha}\right)\left\|v_{1}-v_{2}\right\|_{X_{s, b}^{T}} .
$$

As a result, by increasing the constants $C_{2}$ and $C_{3}$, we obtain

$$
\|\Gamma(v)\|_{X_{s, b}^{T}} \leq C_{1}\left(\|\phi\|_{s}+\|\psi\|_{s}\right)+C_{2}\|v\|_{X_{s, b}^{T}}^{\alpha+1}
$$

for any $v \in X_{s, b}^{T}$ and

$$
\left\|\Gamma\left(v_{1}\right)-\Gamma\left(v_{2}\right)\right\|_{X_{s, b}^{T}} \leq C_{3}\left(\left\|v_{1}\right\|_{X_{s, b}^{T}}^{\alpha}+\left\|v_{2}\right\|_{X_{s, b}^{T}}^{\alpha}\right)\left\|v_{1}-v_{2}\right\|_{X_{s, b}^{T}}
$$

for any $v_{1}, v_{2} \in X_{s, b}^{T}$. Pick $\delta>0, \phi, \psi \in H^{s}\left(\mathbb{T}^{n}\right)$ with $\|\phi\|_{s}+\|\psi\|_{s} \leq \delta$, and set $M=2 C_{1} \delta$. If $\|v\|_{X_{s, b}^{T}} \leq M$ and

$$
\left\|v_{j}\right\|_{X_{s, b}^{T}} \leq M, j=1,2
$$

then

$$
\begin{aligned}
\|\Gamma(v)\|_{X_{s, b}^{T}} & \leq C_{1} \delta+C_{2} M^{\alpha+1} \\
& \leq 2 C_{1} \delta=M
\end{aligned}
$$

as long as

$$
C_{2} M^{\alpha} \leq \frac{1}{2}
$$


Choose $\delta>0$ so that $M=2 C_{1} \delta$ fulfills

$$
C_{2} M^{\alpha} \leq \frac{1}{2} \text { and } C_{3} M^{\alpha} \leq \frac{1}{4}
$$

and let $B_{M}$ be the ball in the space $X_{s, b}^{T}$ centered at the origin of radius $M$. For given $\phi, \psi \in H^{s}\left(\mathbb{T}^{n}\right)$ with $\|\phi\|_{s}+\|\psi\|_{s} \leq \delta$, we have

$$
\|\Gamma(v)\|_{X_{s, b}^{T}} \leq M
$$

for any $v \in B_{M}$ and

$$
\left\|\Gamma\left(v_{1}\right)-\Gamma\left(v_{2}\right)\right\|_{X_{s, b}^{T}} \leq \frac{1}{2}\left\|v_{1}-v_{2}\right\|_{X_{s, b}^{T}}
$$

for any $v_{1}, v_{2} \in B_{M}$. That is to say, $\Gamma$ is a contraction in the ball $B_{M}$. The proof is complete.

Let us now consider the Schrödinger equation posed on a cube $\Omega=(0, \pi)^{n}$

$$
i u_{t}+\Delta u+N(u)=i a(x) h(x, t), \quad x \in \Omega, t \in(0, T)
$$

with either the homogeneous Dirichlet boundary conditions

$$
u(x, t)=0 \quad(x, t) \in \partial \Omega \times(0, T)
$$

or the homogeneous Neumann boundary conditions

$$
\frac{\partial u}{\partial \nu}(x, t)=0 \quad(x, t) \in \partial \Omega \times(0, T) .
$$

The nonlinearity $N(u)$ is still as in (61).

It is remarkable that internal control results with Dirichlet (resp. Neumann) homogeneous boundary conditions can be deduced from those already proved for periodic boundary conditions.

Corollary 3.7 For given $n \geq 2, \alpha_{1}, \alpha_{2} \in \mathbb{N}$ with $\alpha_{1}+\alpha_{2}=: \alpha+1 \geq 2$ and $\alpha$ even, and a $\not \equiv$, the system (60) $-(67)$ is locally exactly controllable in the space $H_{D}^{s}(\Omega)$ for any $s>s_{\alpha, n}$. More precisely, for any given $T>0$, there exists a number $\delta>0$ depending on $\alpha, n, T$ and $\lambda$ such that if $\phi, \psi \in H_{D}^{s}(\Omega)$ satisfy

$$
\|\phi\|_{H_{D}^{s}(\Omega)} \leq \delta, \quad\|\psi\|_{H_{D}^{s}(\Omega)} \leq \delta
$$

then one can choose a control input $h \in L^{2}\left(0, T ; H_{D}^{s}(\Omega)\right)$ such that the system (69)-(67) admits a solution $u \in C\left([0, T] ; H_{D}^{s}(\Omega)\right)$ satisfying

$$
u(x, 0)=\phi(x), \quad u(x, T)=\psi(x) .
$$

Corollary 3.8 For given $n \geq 2, \alpha_{1}, \alpha_{2} \in \mathbb{N}$ with $\alpha_{1}+\alpha_{2}=: \alpha+1 \geq 2$ and $a \neq 0$, the system (66)-(68) is locally exactly controllable in the space $H_{N}^{s}(\Omega)$ for any $s>s_{\alpha, n}$. More precisely, for any given $T>0$, there exists a number $\delta>0$ depending on $\alpha, n, T$ and $\lambda$ such that if $\phi, \psi \in H_{N}^{s}(\Omega)$ satisfy

$$
\|\phi\|_{H_{N}^{s}(\Omega)} \leq \delta, \quad\|\psi\|_{H_{N}^{s}(\Omega)} \leq \delta
$$

then one can choose a control input $h \in L^{2}\left(0, T ; H_{N}^{s}(\Omega)\right)$ such that the system (60)-(68) admits a solution $u \in C\left([0, T] ; H_{N}^{s}(\Omega)\right)$ satisfying

$$
u(x, 0)=\phi(x), \quad u(x, T)=\psi(x) .
$$

We shall say that a function from $(-\pi, \pi)^{n}$ to $\mathbb{C}$ is odd (resp. even), if it is odd with respect to each coordinate $x_{i}, 1 \leq i \leq n$. The proof relies on the basic, but crucial observation that the functions in $H_{D}^{s}(\Omega)$ (resp. $\left.H_{N}^{s}(\Omega)\right)$ coincide with the restrictions to $\Omega$ of the functions in $H^{s}\left(\mathbb{T}^{n}\right)$ which are odd (resp. even). The issue is therefore reduced to an extension of Theorem 3.1 in the framework of odd (resp. even) functions in $H^{s}\left(\mathbb{T}^{n}\right)$. Extending the function $a$ in $(66)$ to $\mathbb{T}^{n}$ as an even function, we notice that the control input $h$ in Theorem 2.2 can be chosen odd (resp. even) if the functions $\phi, \psi$ are odd (resp. even). Indeed, the observability inequality holds as well in the subspaces

$$
\begin{aligned}
H_{\text {odd }}^{s}\left(\mathbb{T}^{n}\right) & =\left\{u \in H_{p}^{s}\left(\mathbb{T}^{n}\right) ; u\left(x_{1}, \ldots, x_{i-1},-x_{i}, x_{i+1}, \ldots, x_{n}\right)=-u(x) \quad \forall x \in \mathbb{T}^{n}, \forall i\right\}, \\
H_{\text {even }}^{s}\left(\mathbb{T}^{n}\right) & =\left\{u \in H_{p}^{s}\left(\mathbb{T}^{n}\right) ; u\left(x_{1}, \ldots, x_{i-1},-x_{i}, x_{i+1}, \ldots, x_{n}\right)=u(x) \quad \forall x \in \mathbb{T}^{n}, \forall i\right\}
\end{aligned}
$$

of $H^{s}\left(\mathbb{T}^{n}\right)$ for $s \leq 0$. On the other hand, since $u$ and $N(u)$ are simultaneously odd (resp. even), we see that the contraction mapping theorem can be applied in a space of odd (resp. even) trajectories to derive the result in Corollary 3.7 (resp. 3.8). Full details are provided in 46 for $n=1$. 


\subsection{Boundary control}

In this section we consider the Schrödinger equation posed on a rectangle $\Omega=\left(0, l_{1}\right) \times \cdots \times\left(0, l_{n}\right)$

$$
i u_{t}+\Delta u+N(u)=0, \quad x \in \Omega, t \in(0, T)
$$

with either the Dirichlet boundary conditions

$$
u(x, t)=1_{\Gamma_{0}} h(x, t) \quad(x, t) \in \partial \Omega \times(0, T)
$$

or the Neumann boundary conditions

$$
\frac{\partial u}{\partial \nu}(x, t)=1_{\Gamma_{0}} h(x, t) \quad(x, t) \in \partial \Omega \times(0, T) .
$$

When we shall consider a smooth Dirichlet controller $g$, then the boundary condition (70) will be replaced by

$$
u(x, t)=g(x) h(x, t) \quad(x, t) \in \partial \Omega \times(0, T) .
$$

$N(u)$ still stands for the nonlinear term in NLS. We first give a result (with a small control region) providing precise informations on the smoothness of the control input and of the trajectories when $N(u)$ is weakly nonlinear. To simplify the exposition, we assume here that

$$
\Omega=(0, \pi)^{n} .
$$

We denote by $u=W_{D}(t) u_{0}$ the solution of (20) for $h=0$. For given $s, b \in \mathbb{R}, X_{s, b}(\Omega)$ denotes the Bourgain space of functions $u: \Omega \times \mathbb{R} \rightarrow \mathbb{C}$ for which the norm

$$
\|u\|_{X_{s, b}(\Omega)}=c\left\|W_{D}(-t) u(., t)\right\|_{H^{b}\left(\mathbb{R} ; H_{D}^{s}(\Omega)\right)}
$$

is finite. Decomposing $u$ as

$$
u(x, t)=\sum_{k \in\left(\mathbb{N}^{*}\right)^{n}} \int_{\mathbb{R}} \hat{u}(k, \tau) e^{i \tau t} \sin \left(k_{1} x_{1}\right) \cdots \sin \left(k_{n} x_{n}\right) d \tau
$$

we can choose the constant $c$ so that

$$
\|u\|_{X_{s, b}(\Omega)}^{2}=\sum_{k \in\left(\mathbb{N}^{*}\right)^{n}} \int_{\mathbb{R}}\left\langle\tau+|k|^{2}\right\rangle^{2 b}\langle k\rangle^{2 s}|\hat{u}(k, \tau)|^{2} d \tau<\infty .
$$

The restriction norm space $X_{s, b}^{T}(\Omega)$ is defined in the usual way (see above the definition of $X_{s, b}^{T}$ ). For $u \in H_{D}^{s}(\Omega)$ given, we denote by $\tilde{u}$ its odd extension to $\mathbb{T}^{n}=(-\pi, \pi)^{n}$; i.e., $\tilde{u}_{\mid(0, \pi)^{n}}=u$, and $\tilde{u}$ is odd with respect to each coordinate $x_{i}$. Note that $\tilde{u} \in H^{s}\left(\mathbb{T}^{n}\right)$ and $\|\tilde{u}\|_{s} \sim\|u\|_{H_{D}^{s}(\Omega)}$. Defining $\tilde{u}(., t)$ from $u(., t)$ in a similar way, we observe that

$$
\|\tilde{u}\|_{X_{s, b}^{T}} \sim\|u\|_{X_{s, b}^{T}(\Omega)}
$$

It is then clear that Lemmas 3.2 and 3.3 hold true with $W_{D}(t), H_{D}^{s}(\Omega)$ and $X_{s, b}^{T}(\Omega)$ substituted to $W(t)$, $H^{s}\left(\mathbb{T}^{n}\right)$ and $X_{s, b}^{T}$, respectively. We shall assume that the nonlinear term $N(u)$ satisfies the following multilinear estimate

$$
\|N(u)-N(v)\|_{X_{s, b^{\prime}}(\Omega)} \leq c(u, v)\|u-v\|_{X_{s, b}(\Omega)}
$$

where $s \in \mathbb{R},-1 / 2<b^{\prime}<b \leq b^{\prime}+1$ and $c(u, v) \rightarrow 0$ as $u \rightarrow 0, v \rightarrow 0$ in $X_{s, b}(\Omega)$.

Theorem 2.11 can be extended to a semilinear context as follows.

Theorem 3.9 Let $g$ be a smooth Dirichlet controller, and let the nonlinearity $N(u)$ satisfy (61) and (73) with $s \in\left[-1, \frac{1}{2}\right), b>0$ and $s+2 b<\frac{1}{2}$. Pick any $T>0$. Then there exists $\delta>0$ such that for any $u_{0}, u_{T} \in H_{D}^{s}(\Omega)$ satisfying

$$
\left\|u_{0}\right\|_{H_{D}^{s}(\Omega)} \leq \delta, \quad\left\|u_{T}\right\|_{H_{D}^{s}(\Omega)} \leq \delta
$$

one may find a control input $h \in H^{\frac{s+1}{2}}\left(\mathbb{T} ; L^{2}(\partial \Omega)\right)$ and a solution $u \in C\left([0, T] ; H_{D}^{s}(\Omega)\right) \cap X_{s, b}^{T}$ of $(69)$ and (72) such that $u(0)=u_{0}$ and $u(T)=u_{T}$. 
Proof. For $u_{T} \in H_{D}^{s}(\Omega)$, let $h$ be the control given by HUM which steers (32) from 0 to $u_{T}$, namely $h=$ $\partial v / \partial \nu$ with $v=W_{D}(t) v_{0}$ and $v_{0}=S^{-1} u_{T} \in H_{D}^{s+2}(\Omega)$ (cf. Theorem 2.11). Recall that $h \in H^{\frac{s+1}{2}}\left(\mathbb{T} ; L^{2}(\partial \Omega)\right.$ ) by (35). We set $u=\Lambda u_{T}=\Gamma S^{-1} u_{T}$. The regularity of $u$ is depicted in the following proposition.

Proposition 3.10 Assume that $-1 \leq s<1 / 2$ and $s+2 b<1 / 2$. Then $\Lambda$ maps continuously $H_{D}^{s}(\Omega)$ into $C\left([0, T] ; H_{D}^{s}(\Omega)\right) \cap X_{s, b}^{T}(\Omega)$.

Proof of Proposition 3.10. It follows from Proposition 2.12 and Theorem 2.11 that $\Lambda$ maps continuously $H_{D}^{s}(\Omega)$ into $C\left([0, T] ; H_{D}^{s}(\Omega)\right)$. Let us turn our attention to the Bourgain space $X_{s, b}^{T}(\Omega)$.

Step 1. We prove several claims used thereafter.

Claim 3. For any $\gamma>1 / 2$, it holds

$$
\sup _{\lambda \in \mathbb{R}} \sum_{k \in \mathbb{Z}}\left\langle\lambda^{2}-k^{2}\right\rangle^{-\gamma}<\infty .
$$

In what follows, $C$ denotes a constant independent of $\lambda$ and $k$ which may vary from line to line. Pick $\lambda \in \mathbb{R}^{+}$. For $0 \leq \lambda \leq 1$

$$
\left\langle\lambda^{2}-k^{2}\right\rangle^{-\gamma} \leq\left\langle k^{2}\right\rangle^{-\gamma}+\left\langle 1-k^{2}\right\rangle^{-\gamma}
$$

and the result is then obvious. For $\lambda>1$, we have

$$
\begin{aligned}
\sum_{k \in \mathbb{Z}}\left\langle\lambda^{2}-k^{2}\right\rangle^{-\gamma} & \leq C\left(\int_{0}^{\lambda-1}\left|\lambda^{2}-x^{2}\right|^{-\gamma} d x+\int_{\lambda+1}^{\infty}\left|x^{2}-\lambda^{2}\right|^{-\gamma} d x+1\right) \\
& =C \lambda^{1-2 \gamma}\left(\int_{0}^{1-\lambda^{-1}}\left|1-y^{2}\right|^{-\gamma} d y+\int_{1+\lambda^{-1}}^{+\infty}\left|y^{2}-1\right|^{-\gamma} d y+1\right) \\
& \leq C \lambda^{1-2 \gamma}\left(\int_{0}^{1-\lambda^{-1}}|1-y|^{-\gamma} d y+\int_{1+\lambda^{-1}}^{2}|y-1|^{-\gamma} d y+1\right) \\
& \leq \begin{cases}C \lambda^{1-2 \gamma}\left(\lambda^{-1+\gamma}+1\right) & \text { if } \gamma \neq 1 \\
C \lambda^{-1}(\ln \lambda+1) & \text { if } \gamma=1\end{cases}
\end{aligned}
$$

and the claim follows.

Claim 4. If $s \geq-1,0<\delta<1, s+2 \delta<1 / 2$, and $k>1+2(s+1)$, then for some constant $C>0$

$$
S(p):=\sum_{q ;|q| \neq|p|} \frac{q_{n}^{2 s+2}}{\left.|| q\right|^{2}-\left.|p|^{2}\right|^{2(1-\delta)}} \prod_{j=1}^{n-1}\left\langle p_{j}-q_{j}\right\rangle^{-k} \leq C\langle p\rangle^{2 s+2} .
$$

Write $S(p)=S^{1}(p)+S^{2}(p)$, where the sum $S^{1}(p)$ is restricted to the $q=\left(q^{\prime}, q_{n}\right)$ with $\left|q^{\prime}\right| \geq|p|$ and $|q| \neq|p|$. Noticing that $|q|^{2}-|p|^{2}=q_{n}^{2}+\left|q^{\prime}\right|^{2}-|p|^{2} \geq q_{n}^{2}$ for such $q$, we obtain that

$$
S^{1}(p) \leq \sum_{q_{n}} q_{n}^{2 s+4 \delta-2} \sum_{q^{\prime}} \prod_{j=1}^{n-1}\left\langle p_{j}-q_{j}\right\rangle^{-k} \leq C \leq C\langle p\rangle^{2 s+2}
$$

To bound $S^{2}(p)$, we fix any $q^{\prime} \in\left(\mathbb{N}^{*}\right)^{n-1}$ with $\left|q^{\prime}\right|<|p|$ and set

$$
\lambda=\sqrt{|p|^{2}-\left|q^{\prime}\right|^{2}} \geq 1
$$

We have that

$$
\begin{aligned}
\sum_{q_{n} ;\left|q_{n}^{2}-\lambda^{2}\right| \geq 1} \frac{q_{n}^{2 s+2}}{\left|q_{n}^{2}-\lambda^{2}\right|^{2(1-\delta)}} & \leq C\left(\int_{\left|x^{2}-\lambda^{2}\right| \geq 1} \frac{x^{2 s+2}}{\left|x^{2}-\lambda^{2}\right|^{2(1-\delta)}} d x+\lambda^{2 s+2}\right) \\
& \leq C\left(\lambda^{2 s+4 \delta-1} \int_{\left|y^{2}-1\right| \geq \lambda^{-2}} \frac{y^{2 s+2}}{\left|y^{2}-1\right|^{2(1-\delta)}} d y+\lambda^{2 s+2}\right) \\
& \leq C\left(\lambda^{2 s+4 \delta-1} \cdot \lambda^{2-4 \delta} \cdot \ln \lambda+\lambda^{2 s+2}\right) \\
& \leq C\left(p_{n}^{2 s+2}+\sum_{j=1}^{n-1}\left\langle p_{j}^{2}-q_{j}^{2}\right\rangle^{s+1}\right) .
\end{aligned}
$$


It follows that

$$
\begin{aligned}
S^{2}(p) & \leq C \sum_{q^{\prime}}\left(p_{n}^{2 s+2}+\sum_{j=1}^{n-1}\left\langle p_{j}^{2}-q_{j}^{2}\right\rangle^{s+1}\right) \prod_{l=1}^{n-1}\left\langle p_{l}-q_{l}\right\rangle^{-k} \\
& \leq C\left(p_{n}^{2 s+2}+\sum_{j=1}^{n-1} \sum_{q_{j} \geq 1}\left\langle p_{j}^{2}-q_{j}^{2}\right\rangle^{s+1}\left\langle p_{j}-q_{j}\right\rangle^{-k}\right) \\
& \leq C\left(p_{n}^{2 s+2}+\sum_{j=1}^{n-1} \sum_{q_{j} \geq 1}\left\langle p_{j}+q_{j}\right\rangle^{s+1}\left\langle q_{j}-p_{j}\right\rangle^{-(k-s-1)}\right) .
\end{aligned}
$$

To complete the proof of Claim 4, we need the following

Claim 5. Let $\sigma \geq 0$ and $k>\sigma+1$. Then there exists a constant $C>0$ such that

$$
\sum_{m \geq 1}\langle m+n\rangle^{\sigma}\langle m-n\rangle^{-k} \leq C n^{\sigma} \quad \forall n \geq 1 .
$$

Split the sum into $\Sigma_{1}+\Sigma_{2}$ where $\Sigma_{1}=\sum_{1 \leq m \leq 3 n}\langle m+n\rangle^{\sigma}\langle m-n\rangle^{-k}$. Note that

$$
\Sigma_{1} \leq\langle 4 n\rangle^{\sigma} \sum_{l \in \mathbb{Z}}\langle l\rangle^{-k} \leq C\langle n\rangle^{\sigma}
$$

since $k>1$. On the other hand, noticing that $m-n>(m+n) / 2$ for $m>3 n$, we have that

$$
\Sigma_{2} \leq \sum_{m>3 n}\langle 2(m-n)\rangle^{\sigma}\langle m-n\rangle^{-k} \leq C \sum_{m>3 n}\langle m-n\rangle^{-(k-\sigma)} \leq C .
$$

Claim 5 is proved. Pick $k>1+2(s+1) \geq 1$. It follows from Claim 5 that

$$
\sum_{q_{j}}\left\langle p_{j}+q_{j}\right\rangle^{s+1}\left\langle p_{j}-q_{j}\right\rangle^{-(k-s-1)} \leq C p_{j}^{s+1}
$$

Since $s+1 \geq 0$ and $p_{j} \geq 1$, we conclude that

$$
S(p) \leq C\left(p_{n}^{2 s+2}+\left\langle p^{\prime}\right\rangle^{s+1}\right) \leq C\langle p\rangle^{2 s+2} .
$$

This completes the proof of Claim 4.

Step 2. Assume that $s<0$ and $s+2 b<1 / 2$, and pick any $u_{T} \in H_{P}^{s}(\Omega)$ and any $\eta \in C_{0}^{\infty}(\mathbb{R})$ with $\eta(t)=1$ for $0 \leq t \leq T$. Let $v_{0}=S^{-1} u_{T} \in H_{D}^{s+2}(\Omega)$ be decomposed as in (34). Let us prove that $u=\Lambda u_{T} \in X_{s, b}^{T}$. It is sufficient to prove that

$$
\|\eta(t) u\|_{X_{s, b}} \leq C\left\|v_{0}\right\|_{H_{D}^{s+2}(\Omega)} .
$$

Recall that $u$ is given by (46)-(47), and that $u(t)$ may be defined this way for all $t \in \mathbb{R}$. Again, we can limit ourselves to proving that $u_{F_{0}} \in X_{s, b}^{T}$, where $u_{F_{0}}$ is the contribution due to $F_{0}=\left\{x \in \partial \Omega ; x_{n}=0\right\}$ in $u$. $u_{F_{0}}$ is decomposed as

$$
u_{F_{0}}=\sum_{q \in\left(\mathbb{N}^{*}\right)^{n}} u_{q}(t) \sin \left(q_{1} x_{1}\right) \cdots \sin \left(q_{n} x_{n}\right)
$$

where

$$
u_{q}(t)=-\left(\frac{2}{\pi}\right)^{n} \sum_{p \in\left(\mathbb{N}^{*}\right)^{n}} v_{p} \frac{e^{-i|p|^{2} t}-e^{-i|q|^{2} t}}{|q|^{2}-|p|^{2}} I_{F_{0}}
$$

with the convention (40). . denoting time Fourier transform, an application of the elementary property

$$
\widehat{e^{i r t} \eta(t)}(\tau)=\hat{\eta}(\tau-r)
$$

yields

$$
\widehat{\eta u_{q}}(\tau)=-\left(\frac{2}{\pi}\right)^{n}\left(\sum_{p ;|p| \neq|q|} v_{p} \frac{\hat{\eta}\left(\tau+|p|^{2}\right)-\hat{\eta}\left(\tau+|q|^{2}\right)}{|q|^{2}-|p|^{2}} I_{F_{0}}+\sum_{p ;|p|=|q|} i v_{p} \widehat{\operatorname{t\eta }(t)}\left(\tau+|q|^{2}\right) I_{F_{0}}\right) .
$$


For a function $w$ decomposed as

$$
w(x, t)=\sum_{q \in\left(\mathbb{N}^{*}\right)^{n}} w_{q}(t) \sin \left(q_{1} x_{1}\right) \cdots \sin \left(q_{n} x_{n}\right)
$$

we recall that

$$
\|w\|_{X_{s, b}(\Omega)}^{2}=\sum_{q \in\left(\mathbb{N}^{*}\right)^{n}} \int d \tau\left\langle\tau+|q|^{2}\right\rangle^{2 b}\langle q\rangle^{2 s}\left|\hat{w}_{q}(\tau)\right|^{2}
$$

Therefore, it is sufficient to check that

$$
I:=\sum_{q \in\left(\mathbb{N}^{*}\right)^{n}} \int d \tau\langle q\rangle^{2 s}\left\langle\tau+|q|^{2}\right\rangle^{2 b}\left|\widehat{\eta u_{q}}(\tau)\right|^{2} \leq c \sum_{p}\langle p\rangle^{2 s+4}\left|v_{p}\right|^{2} .
$$

Using (42), we may write

$$
I \leq c\left(I_{1}+I_{2}+I_{3}\right)
$$

where

$$
\begin{aligned}
I_{1} & =\sum_{q} \int d \tau\langle q\rangle^{2 s}\left\langle\tau+|q|^{2}\right\rangle^{2 b}\left(\sum_{p ;|p|=|q|}\left|v_{p} \widehat{\operatorname{t\eta }(t)}\left(\tau+|q|^{2}\right)\right| p_{n} q_{n} \prod_{j=1}^{n-1}\left\langle p_{j}-q_{j}\right\rangle^{-k}\right)^{2} \\
I_{2} & =\sum_{q} \int d \tau\langle q\rangle^{2 s}\left\langle\tau+|q|^{2}\right\rangle^{2 b}\left(\sum_{p ;|p| \neq|q|}\left|v_{p} \frac{\hat{\eta}\left(\tau+|q|^{2}\right)}{|q|^{2}-|p|^{2}}\right| p_{n} q_{n} \prod_{j=1}^{n-1}\left\langle p_{j}-q_{j}\right\rangle^{-k}\right)^{2} \\
I_{3} & =\sum_{q} \int d \tau\langle q\rangle^{2 s}\left\langle\tau+|q|^{2}\right\rangle^{2 b}\left(\sum_{p ;|p| \neq|q|}\left|v_{p} \frac{\hat{\eta}\left(\tau+|p|^{2}\right)}{|q|^{2}-|p|^{2}}\right| p_{n} q_{n} \prod_{j=1}^{n-1}\left\langle p_{j}-q_{j}\right\rangle^{-k}\right)^{2}
\end{aligned}
$$

We bound separately $I_{1}, I_{2}$ and $I_{3}$. 1 .

$$
\begin{aligned}
I_{1} & \leq C\left(\int d \sigma\langle\sigma\rangle^{2 b}|\widehat{\operatorname{t\eta }(t)}(\sigma)|^{2}\right) \sum_{q}\langle q\rangle^{2 s} q_{n}^{2}\left(\sum_{p ;|p|=|q|}\left|v_{p}\right| p_{n} \prod_{j=1}^{n-1}\left\langle p_{j}-q_{j}\right\rangle^{-k}\right)^{2} \\
& \leq C \sum_{q}\langle q\rangle^{2 s} q_{n}^{2}\left(\sum_{p ;|p|=|q|}\left|v_{p}\right|^{2} p_{n}^{2} \prod_{j=1}^{n-1}\left\langle p_{j}-q_{j}\right\rangle^{-k}\right)\left(\sum_{p ;|p|=|q|} \prod_{j=1}^{n-1}\left\langle p_{j}-q_{j}\right\rangle^{-k}\right)
\end{aligned}
$$

where we used successively a change of variables in the integral term, the fact that $\eta \in \mathcal{S}(\mathbb{R})$ and CauchySchwarz inequality. From

$$
\sum_{p ;|p|=|q|} \prod_{j=1}^{n-1}\left\langle p_{j}-q_{j}\right\rangle^{-k} \leq \sum_{p_{1}, \ldots, p_{n-1}}\left(\prod_{j=1}^{n-1}\left\langle p_{j}-q_{j}\right\rangle^{-k} \sum_{p_{n} ;|p|=|q|} 1\right) \leq \prod_{j=1}^{n-1} \sum_{p_{j} \in \mathbb{Z}}\left\langle p_{j}\right\rangle^{-k}<\infty
$$

we deduce that

$$
\begin{aligned}
I_{1} & \leq C \sum_{p}\left|v_{p}\right|^{2}|p|^{2} \sum_{q ;|q|=|p|}\langle q\rangle^{2 s+2} \prod_{j=1}^{n-1}\left\langle p_{j}-q_{j}\right\rangle^{-k} \\
& \leq C \sum_{p}\left|v_{p}\right|^{2}|p|^{2 s+4}
\end{aligned}
$$

2.

$$
\begin{aligned}
I_{2} & =C\left(\int d \sigma\langle\sigma\rangle^{2 b}|\hat{\eta}(\sigma)|^{2}\right) \sum_{q}\langle q\rangle^{2 s} q_{n}^{2}\left(\sum_{p ;|p| \neq|q|}\left|\frac{v_{p}}{|q|^{2}-|p|^{2}}\right| p_{n} \prod_{j=1}^{n-1}\left\langle p_{j}-q_{j}\right\rangle^{-k}\right)^{2} \\
& \leq c \sum_{q}\langle q\rangle^{2 s} q_{n}^{2}\left(\sum_{p ;|p| \neq|q|} \frac{\left|v_{p}\right|^{2} p_{n}^{2}}{\left.|| q\right|^{2}-\left.|p|^{2}\right|^{2(1-\delta)}} \prod_{j=1}^{n-1}\left\langle p_{j}-q_{j}\right\rangle^{-k}\right)\left(\left.\sum_{p ;|p| \neq|q|}|| q\right|^{2}-\left.|p|^{2}\right|^{-2 \delta} \prod_{j=1}^{n-1}\left\langle p_{j}-q_{j}\right\rangle^{-k}\right)
\end{aligned}
$$


where we used Cauchy-Schwarz inequality, and $\delta>1 / 4$ was chosen so that $s+2 \delta<1 / 2$. From Claim 3 , we obtain that

$$
\left.\sum_{p ;|p| \neq|q|}|| q\right|^{2}-\left.|p|^{2}\right|^{-2 \delta} \prod_{j=1}^{n-1}\left\langle p_{j}-q_{j}\right\rangle^{-k} \leq C \sum_{p^{\prime}} \prod_{j=1}^{n-1}\left\langle p_{j}-q_{j}\right\rangle^{-k} \sum_{p_{n} ;|p| \neq|q|}\left\langle|q|^{2}-|p|^{2}\right\rangle^{-2 \delta}<\text { const. }
$$

Therefore, since $s<0$, we see that

$$
I_{2} \leq C \sum_{q} q_{n}^{2 s+2} \sum_{p ;|p| \neq|q|} \frac{\left|v_{p}\right|^{2} p_{n}^{2}}{\left.|| q\right|^{2}-\left.|p|^{2}\right|^{2(1-\delta)}} \sum_{j=1}^{n-1}\left\langle p_{j}-q_{j}\right\rangle^{-k}
$$

and from Claim 4

$$
I_{2} \leq C \sum_{p}\left|v_{p}\right|^{2}|p|^{2 s+4}
$$

3. From the elementary estimate

$$
\left\langle\tau+|q|^{2}\right\rangle \leq c\left\langle\tau+|p|^{2}\right\rangle\left\langle|q|^{2}-|p|^{2}\right\rangle
$$

we infer that

$$
I_{3} \leq C \sum_{q} \int d \tau\langle q\rangle^{2 s}\left|q_{n}\right|^{2}\left(\sum_{p ;|p| \neq|q|}\left|v_{p}\right| \frac{\left|\hat{\eta}\left(\tau+|p|^{2}\right)\right|\left\langle\tau+|p|^{2}\right\rangle^{b}}{\left.|| q\right|^{2}-\left.|p|^{2}\right|^{1-b}} p_{n} \prod_{j=1}^{n-1}\left\langle p_{j}-q_{j}\right\rangle^{-k}\right)^{2} .
$$

For any fixed $\gamma>1$, we have that for some constant $c>0$

$$
\langle\sigma\rangle^{b}|\hat{\eta}(\sigma)| \leq c\langle\sigma\rangle^{-\gamma} \quad \forall \sigma \in \mathbb{R}
$$

Expanding the squared term in (74) results in

$$
\begin{aligned}
I_{3} \leq & C \sum_{q}\langle q\rangle^{2 s}\left|q_{n}\right|^{2} \sum_{p ;|p| \neq|q|} \sum_{\tilde{p} ;|\tilde{p}| \neq|q|} \frac{\left|v_{p}\right|\left|v_{\tilde{p}}\right| p_{n} \tilde{p}_{n}}{\left.|| q\right|^{2}-\left.\left.|p|^{2}\right|^{1-b}|| q\right|^{2}-\left.|\tilde{p}|^{2}\right|^{1-b}} \\
& \times\left(\prod_{j=1}^{n-1}\left\langle p_{j}-q_{j}\right\rangle^{-k}\left\langle\tilde{p}_{j}-q_{j}\right\rangle^{-k}\right) \int d \tau\left\langle\tau+|p|^{2}\right\rangle^{-\gamma}\left\langle\tau+|\tilde{p}|^{2}\right\rangle^{-\gamma} \\
\leq & C \sum_{q}\langle q\rangle^{2 s}\left|q_{n}\right|^{2} \sum_{p ;|p| \neq|q|} \sum_{\tilde{p} ;|\tilde{p}| \neq|q|} \frac{\left|v_{p}\right|\left|v_{\tilde{p}}\right| p_{n} \tilde{p}_{n}}{\left.|| q\right|^{2}-\left.\left.|p|^{2}\right|^{1-b}|| q\right|^{2}-\left.|\tilde{p}|^{2}\right|^{1-b}} \\
& \times\left(\prod_{j=1}^{n-1}\left\langle p_{j}-q_{j}\right\rangle^{-k}\left\langle\tilde{p}_{j}-q_{j}\right\rangle^{-k}\right)\left\langle|p|^{2}-|\tilde{p}|^{2}\right\rangle^{-\gamma}
\end{aligned}
$$

where we used the following estimate valid for $\gamma>1$ (see e.g. [33, Lemma 7.34])

$$
\int d \tau\left\langle\tau+\tau_{1}\right\rangle^{-\gamma}\left\langle\tau+\tau_{2}\right\rangle^{-\gamma} \leq c\left\langle\tau_{1}-\tau_{2}\right\rangle^{-\gamma}
$$

Thus

$$
I_{3} \leq C \sum_{q}\langle q\rangle^{2 s} q_{n}^{2} \sum_{p ;|p| \neq|q|} \frac{\left|v_{p}\right|^{2} p_{n}^{2}}{\left.|| q\right|^{2}-\left.|p|^{2}\right|^{2(1-b)}}\left(\prod_{j=1}^{n-1}\left\langle p_{j}-q_{j}\right\rangle^{-k}\right) \sum_{\tilde{p} ;|\tilde{p}| \neq|q|} \prod_{j=1}^{n-1}\left\langle\tilde{p}_{j}-q_{j}\right\rangle^{-k}\left\langle|p|^{2}-|\tilde{p}|^{2}\right\rangle^{-\gamma} .
$$

Since $\gamma>1 / 2$, it follows from Claim 3 that

$$
\sum_{\tilde{p}} \prod_{j=1}^{n-1}\left\langle\tilde{p}_{j}-q_{j}\right\rangle^{-k}\left\langle|p|^{2}-|\tilde{p}|\right\rangle^{-\gamma} \leq \sum_{\tilde{p}_{1}, \ldots, \tilde{p}_{n-1}} \prod_{j=1}^{n-1}\left\langle\tilde{p}_{j}-q_{j}\right\rangle^{-k} \sum_{\tilde{p}_{n}}\left\langle\tilde{p}_{n}^{2}+\left|\tilde{p}^{\prime}\right|^{2}-|p|^{2}\right\rangle^{-\gamma}<\text { const. }
$$


Thus

$$
I_{3} \leq C \sum_{q}\langle q\rangle^{2 s} q_{n}^{2} \sum_{p ;|p| \neq|q|} \frac{\left|v_{p}\right|^{2} p_{n}^{2}}{\left.|| q\right|^{2}-\left.|p|^{2}\right|^{2(1-b)}} \prod_{j=1}^{n-1}\left\langle p_{j}-q_{j}\right\rangle^{-k} .
$$

Using Claim 4 and the fact that $s \in[-1,0)$, we have that

$$
I_{3} \leq C \sum_{p}\left|v_{p}\right|^{2}|p|^{2} \sum_{q ;|q| \neq|p|} \frac{q_{n}^{2 s+2}}{\left.|| q\right|^{2}-\left.|p|^{2}\right|^{2(1-b)}} \prod_{j=1}^{n-1}\left\langle p_{j}-q_{j}\right\rangle^{-k} \leq \sum_{p}\left|v_{p}\right|^{2}|p|^{2 s+4} .
$$

Step 3. Assume that $s+2 b<1 / 2$ with $s \in[0,1 / 2)$. Let $u_{T}, v_{0}, u$ and $\eta$ be as in Step 2. Then

$$
\begin{aligned}
\left\|\eta(t) \Gamma v_{0}\right\|_{X_{s, b}} & \leq C\left\|\eta D^{s+1} \Gamma v_{0}\right\|_{X_{-1, b}} \\
& \leq C\left(\left\|\eta(t) \Gamma\left(D^{s+1} v_{0}\right)\right\|_{X_{-1, b}}+\left\|\eta(t)\left[\Gamma, D^{s+1}\right] v_{0}\right\|_{X_{-1, b}}\right) .
\end{aligned}
$$

According to Step 2, the first term in the r.h.s. of (75) is less than $C\left\|D^{s+1} v_{0}\right\|_{1} \leq C\left\|v_{0}\right\| \|_{s+2}$, for $-1+2 b<$ $1 / 2$. The contribution due to $F_{0}=\left\{x \in \partial \Omega ; x_{n}=0\right\}$ in $\left\|\eta(t)\left[\Gamma, D^{s+1}\right] v_{0}\right\|_{-1, b}^{2}$ is estimated by

$$
\begin{aligned}
C_{F_{0}} & \leq \sum_{q} \int d \tau\langle q\rangle^{-2}\left\langle\tau+|q|^{2}\right\rangle^{2 b}\left|\sum_{p ;|p| \neq|q|} v_{p} \frac{|p|^{s+1}-|q|^{s+1}}{|q|^{2}-|p|^{2}}\left(\hat{\eta}\left(\tau+|p|^{2}\right)-\hat{\eta}\left(\tau+|q|^{2}\right)\right) I_{F_{0}}\right|^{2} \\
& \leq C\left(I_{2}^{\prime}+I_{3}^{\prime}\right)
\end{aligned}
$$

where

$$
\begin{aligned}
I_{2}^{\prime} & =\sum_{q} \int d \tau\langle q\rangle^{-2}\left\langle\tau+|q|^{2}\right\rangle^{2 b}\left(\sum_{p ;|p| \neq|q|}\left|v_{p} \hat{\eta}\left(\tau+|q|^{2}\right)\right| \frac{|p|^{s}+|q|^{s}}{|p|+|q|} p_{n} q_{n} \prod_{j=1}^{n-1}\left\langle p_{j}-q_{j}\right\rangle^{-k}\right)^{2} \\
I_{3}^{\prime} & =\sum_{q} \int d \tau\langle q\rangle^{-2}\left\langle\tau+|q|^{2}\right\rangle^{2 b}\left(\sum_{p ;|p| \neq|q|}\left|v_{p} \hat{\eta}\left(\tau+|p|^{2}\right)\right| \frac{|p|^{s}+|q|^{s}}{|p|+|q|} p_{n} q_{n} \prod_{j=1}^{n-1}\left\langle p_{j}-q_{j}\right\rangle^{-k}\right)^{2} .
\end{aligned}
$$

We bound separately $I_{2}^{\prime}$ and $I_{3}^{\prime}$.

1. We have that

$$
\begin{aligned}
I_{2}^{\prime} & \leq C\left(\int d \sigma\langle\sigma\rangle^{2 b}|\hat{\eta}(\sigma)|^{2}\right) \sum_{q}\langle q\rangle^{-2}\left|q_{n}\right|^{2}\left|\sum_{p ;|p| \neq|q|}\right| v_{p}\left|\frac{|p|^{s}+|q|^{s}}{|p|+|q|} p_{n} \prod_{j=1}^{n-1}\left\langle p_{j}-q_{j}\right\rangle^{-k}\right|^{2} \\
& \leq C \sum_{q}\left|\sum_{p}\right| v_{p}\left|\frac{|p|^{s}+|q|^{s}}{|p|+|q|} p_{n} \prod_{j=1}^{n-1}\left\langle p_{j}-q_{j}\right\rangle^{-k}\right|^{2} \\
& \leq C \sum_{p}|p|^{3+2 s}\left|v_{p}\right|^{2} \\
& \leq C\left\|v_{0}\right\|_{s+\frac{3}{2}}^{2}
\end{aligned}
$$

where we used (44)-(45).

2. Doing computations similar to those performed in Step 2, we obtain that

$$
\begin{aligned}
I_{3}^{\prime} & \leq\left. C \sum_{q}\langle q\rangle^{-2} q_{n}^{2} \sum_{p ;|p| \neq|q|}\left|v_{p}\right|^{2} p_{n}^{2} \frac{|p|^{2 s}+|q|^{2 s}}{(|p|+|q|)^{2}}|| q\right|^{2}-\left.|p|^{2}\right|^{2 b} \prod_{j=1}^{n-1}\left\langle p_{j}-q_{j}\right\rangle^{-k} \\
& \leq C \sum_{p}\left|v_{p}\right|^{2}|p|^{2} \sum_{q ;|q| \neq|p|}(|p|+|q|)^{2 s+4 b-2} \prod_{j=1}^{n-1}\left\langle p_{j}-q_{j}\right\rangle^{-k} \\
& \leq C|| v_{0} \|_{1}^{2}
\end{aligned}
$$

where we used the fact that $s+2 b<1 / 2$. Since $s+2 \geq 1$, we finally have that

$$
C_{F_{0}} \leq C\left\|v_{0}\right\|_{H_{D}^{s+2}(\Omega)}^{2}
$$


This completes the proof of Proposition 3.10 .

We can now complete the proof of Theorem 3.9. Let $s, b, u_{0}$ and $u_{T}$ be as in the statement of the theorem. Using Proposition 3.10 and proceeding as in the proof of Theorem 3.1. one can show that the map

$$
\Gamma(v)=W_{D}(t) u_{0}+i \int_{0}^{t} W_{D}(t-\tau) N(v)(\tau) d \tau+\Lambda\left(u_{T}-W_{D}(T) u_{0}-\omega(v, T)\right)
$$

has a fixed-point $\Gamma(v)=v$ in some closed ball $B_{M} \subset X_{s, b}^{T}(\Omega)$ provided that $\left\|u_{0}\right\|_{H_{D}^{s}(\Omega)}+\left\|u_{T}\right\|_{H_{D}^{s}(\Omega)}$ is small enough. Such a trajectory $v$ fulfills all the requirements of Theorem 3.9. In particular, $v \in X_{s, b}^{T}(\Omega) \cap$ $C\left([0, T] ; H_{D}^{s}(\Omega)\right)$. The smoothness of the last term in $(76)$ follows from Proposition 3.10. In (76), we used the notation

$$
\omega(v, T)=i \int_{0}^{T} W_{D}(T-\tau) N(v)(\tau) d \tau .
$$

Note that $\int_{0}^{t} W_{D}(t-\tau) N(v)(\tau) d \tau \in X_{s, b^{\prime}+1}^{T}(\Omega) \subset C\left([0, T] ; H_{D}^{s}(\Omega)\right)$, by Lemma 3.3, (73), and the fact that $b^{\prime}>-1 / 2$. In particular, $\omega(v, T) \in H_{D}^{s}(\Omega)$. The proof of Theorem 3.9 is achieved.

Remark 3.11 (a) Using ideas from [1], it is likely that Theorem 3.9 may be applied when $n \geq 2, \Gamma_{0}$ is a neighborhood of a vertex, and $N(u)=\lambda|u|^{\alpha} u$ with $\alpha>0$ small enough.

(b) The condition $s+2 b<1 / 2$ in Proposition 3.10 is actually sharp. Indeed, let us take $n=1$ and pick any $p \in \mathbb{N}^{*}$ and any $\eta \in \mathcal{S}(\mathbb{R})$ with $|\hat{\eta}(\tau)|>1$ for $-1 \leq \tau \leq 1$. Set $v_{0}(x)=\sin (p x)$ for $x \in \Omega=(0, \pi)$. With $\Gamma_{0}=\{0\}$, we have that $I_{F_{0}}=p q$ with

$$
\widehat{\eta u_{q}}(\tau)= \begin{cases}-\frac{2 i}{\pi} \widehat{t \eta(t)}\left(\tau+p^{2}\right) p^{2} & \text { if } q=p \\ -\frac{2}{\pi} \frac{\hat{\eta}\left(\tau+p^{2}\right)-\hat{\eta}\left(\tau+q^{2}\right)}{q^{2}-p^{2}} p q & \text { if } q \neq p .\end{cases}
$$

Therefore

$$
\begin{aligned}
\frac{\pi^{2}}{4}\|\eta u\|_{X_{s, b}(\Omega)}^{2}= & \int d \tau \sum_{q ; q \neq p}\langle q\rangle^{2 s}\left\langle\tau+q^{2}\right\rangle^{2 b}\left|\frac{\hat{\eta}\left(\tau+p^{2}\right)-\hat{\eta}\left(\tau+q^{2}\right)}{q^{2}-p^{2}}\right|^{2} p^{2} q^{2} \\
& +\left(\int d \tau\left\langle\tau+p^{2}\right\rangle^{2 b}\left|\widehat{t \eta(t)}\left(\tau+p^{2}\right)\right|^{2}\right)\langle p\rangle^{2 s} p^{4} \\
= & \int d \tau \sum_{q ; q \neq p}\langle q\rangle^{2 s}\left\langle\tau+q^{2}\right\rangle^{2 b} \frac{\left|\hat{\eta}\left(\tau+p^{2}\right)\right|^{2}}{\left|q^{2}-p^{2}\right|^{2}} p^{2} q^{2}+J(p)
\end{aligned}
$$

where $|J(p)| \leq C p^{2 s+4} \leq C\left\|v_{0}\right\|_{s+2}^{2}$, according to the estimations of $I_{1}, I_{2}$, and the fact that

$$
\int d \tau\left\langle\tau+q^{2}\right\rangle^{2 b}\left|\hat{\eta}\left(\tau+p^{2}\right) \hat{\eta}\left(\tau+q^{2}\right)\right| d \tau \leq \text { const }<\infty
$$

Since for $q \neq p$

$$
\int d \tau\left\langle\tau+q^{2}\right\rangle^{2 b}\left|\hat{\eta}\left(\tau+p^{2}\right)\right|^{2} \geq \int_{-p^{2}-1}^{-p^{2}+1} d \tau\left\langle\tau+q^{2}\right\rangle^{2 b} \geq C\left|q^{2}-p^{2}\right|^{2 b}
$$

we have that for $s+2 b \geq 1 / 2$,

$$
\int d \tau \sum_{q ; q \neq p}\langle q\rangle^{2 s}\left\langle\tau+q^{2}\right\rangle^{2 b} \frac{\left|\hat{\eta}\left(\tau+p^{2}\right)\right|^{2}}{\left|q^{2}-p^{2}\right|^{2}} p^{2} q^{2} \geq C p^{2} \sum_{q ; q>p}\left|q^{2}-p^{2}\right|^{2 b-2}\langle q\rangle^{2 s} q^{2}=\infty
$$

therefore $\eta u \notin X_{s, b}(\Omega)$. The condition $s+2 b<1 / 2$ seems related to the fact that any smooth function on $\mathbb{T}^{n}$ with nonnull boundary values belongs to the space $H_{D}^{s}(\Omega)$ for $s<1 / 2$ only. Better results will probably require to consider other Bourgain spaces than $X_{s, b}(\Omega)$. 
Corollary 3.12 Let $n=1, \Omega=(0, \pi), \Gamma_{0}=\{0\}$, and let the nonlinear term $N(u)$ satisfy

$$
|N(u)-N(v)| \leq C\left(|u|^{\alpha}+|v|^{\alpha}\right)|u-v|, \quad \forall u, v \in \mathbb{R} .
$$

for some $\alpha \in[0,5 / 4)$. Let $p=\frac{4}{3}(\alpha+1)<3$. Then there exists a number $\delta>0$ such that for any $u_{0}, u_{T} \in L^{2}(\Omega)$ satisfying

$$
\left\|u_{0}\right\|_{L^{2}(\Omega)}<\delta, \quad\left\|u_{T}\right\|_{L^{2}(\Omega)}<\delta
$$

one may find a function $h \in H^{\frac{1}{2}}(0, T)$ and a solution $u \in C\left([0, T] ; L^{2}(\Omega)\right) \cap L^{p}\left(0, T ; L^{p}(\Omega)\right)$ of $(69)-(70)$ such that $u(0)=u_{0}$ and $u(T)=u_{T}$.

For instance, $N_{1}(u)=\lambda|u|^{\alpha} u$ with $0 \leq \alpha<5 / 4$, and $N_{2}(u)$ of the form (61) with $\alpha=1$ are concerned.

Proof. From the classical Strichartz estimate (see e.g. [50])

$$
\|u\|_{L^{4}\left(\mathbb{R} ; L^{4}(\mathbb{T})\right)} \leq C\|u\|_{X_{0, \frac{3}{8}}}
$$

we obtain at once the following estimates involving the spaces $X_{s, b}^{T}(\Omega)$

$$
\begin{aligned}
\|u\|_{L^{4}\left(0, T ; L^{4}(\Omega)\right)} & \leq C\|u\|_{X_{0, \frac{3}{8}}^{T}(\Omega)} \\
\|u\|_{X_{0,-\frac{3}{8}}^{T}(\Omega)} & \leq C\|u\|_{L^{\frac{4}{3}}\left(0, T ; L^{\frac{4}{3}}(\Omega)\right)}
\end{aligned}
$$

Notice that for $v \in L^{p}\left(0, T ; L^{p}(\Omega)\right)$, we have that

$$
\int_{0}^{t} W_{D}(t-\tau) N(v)(\tau) d \tau \in X_{0, \frac{5}{8}}^{T}(\Omega) \subset C\left([0, T] ; L^{2}(\Omega)\right) \cap L^{p}\left(0, T ; L^{p}(\Omega)\right) .
$$

Indeed,

$$
\begin{aligned}
\left\|\int_{0}^{t} W_{D}(t-\tau) N(v)(\tau) d \tau\right\|_{X_{0, \frac{5}{8}}^{T}(\Omega)} & \leq C\|N(v)\|_{X_{0,-\frac{3}{8}}^{T}(\Omega)} \\
& \leq C\|N(v)\|_{L^{\frac{4}{3}}\left(0, T ; L^{\frac{4}{3}}(\Omega)\right)} \\
& \leq C\|v\|_{L^{p}\left(0, T ; L^{p}(\Omega)\right)}^{\alpha+1}<\infty .
\end{aligned}
$$

In particular, $\omega(v, T)=i \int_{0}^{T} W_{D}(T-\tau) N(v)(\tau) d \tau \in L^{2}(\Omega)$. On the other hand, by Proposition 3.10, $\Lambda$ maps continuously $L^{2}(\Omega)$ into $C\left([0, T] ; L^{2}(\Omega)\right) \cap X_{0, b}^{T}(\Omega)$ for any $b<1 / 4$. Interpolating between

$$
X_{0, \frac{3}{8}} \subset L^{4}\left(\mathbb{R} ; L^{4}(\mathbb{T})\right) \quad \text { and } \quad X_{0,0}=L^{2}\left(\mathbb{R} ; L^{2}(\mathbb{T})\right)
$$

we obtain that

$$
X_{0, b} \subset L^{p}\left(\mathbb{R} ; L^{p}(\mathbb{T})\right) \quad \text { for } b=\frac{3}{2}\left(\frac{1}{2}-\frac{1}{p}\right)<\frac{1}{4}
$$

Therefore

$$
\Lambda\left(L^{2}(\Omega)\right) \subset C\left([0, T] ; L^{2}(\Omega)\right) \cap L^{p}\left(0, T ; L^{p}(\Omega)\right)
$$

It follows that the map

$$
\Gamma(v)=W_{D}(t) u_{0}+i \int_{0}^{t} W_{D}(t-\tau) N(v)(\tau) d \tau+\Lambda\left(u_{T}-W_{D}(T) u_{0}-\omega(v, T)\right)
$$

is well defined from $L^{p}\left(0, T ; L^{p}(\Omega)\right)$ into $C\left([0, T] ; L^{2}(\Omega)\right) \cap L^{p}\left(0, T ; L^{p}(\Omega)\right)$. Using the computations above, one readily sees that $\Gamma$ contracts in some ball $B_{M} \subset L^{p}\left(0, T ; L^{p}(\Omega)\right)$, provided that $\left\|u_{0}\right\|_{L^{2}(\Omega)}+\left\|u_{T}\right\|_{L^{2}(\Omega)}$ is small enough.

Corollary 3.13 Theorem 3.9 may be applied when $n=2, \Omega=(0, \pi)^{2}, g$ is a smooth Dirichlet controller, $N(u)=\bar{u}^{2}, s \in\left(-\frac{3}{8},-\frac{1}{3}\right), b \in\left(\frac{3}{8}, \frac{1}{2}\right)$ with $s+2 b<\frac{1}{2}$, and $b^{\prime}>-\frac{1}{2}$ is sufficiently close to $-\frac{1}{2}$.

Corollary 3.13 is a direct consequence of Theorem 3.9 and of the following result, whose proof is postponed in Appendix. 
Proposition 3.14 Let $s \in\left(-\frac{3}{8},-\frac{1}{3}\right)$ and $b \in\left(\frac{3}{8}, \frac{1}{2}\right)$. Then there exists $b^{\prime} \in\left(-\frac{1}{2},-\frac{5}{12}\right)$ and $C>0$ such that

$$
\begin{aligned}
\left\|\bar{v}_{1} \bar{v}_{2}\right\|_{X_{s, b^{\prime}}\left(\mathbb{T}^{2}\right)} \leq C\left\|v_{1}\right\|_{X_{s, b}\left(\mathbb{T}^{2}\right)}\left\|v_{2}\right\|_{X_{s, b}\left(\mathbb{T}^{2}\right)}, \quad \forall v_{1}, v_{2} \in X_{s, b}\left(\mathbb{T}^{2}\right), \\
\left\|\bar{u}_{1} \bar{u}_{2}\right\|_{X_{s, b^{\prime}}(\Omega)} \leq C\left\|u_{1}\right\|_{X_{s, b}(\Omega)}\left\|u_{2}\right\|_{X_{s, b}(\Omega)}, \quad \forall u_{1}, u_{2} \in X_{s, b}(\Omega) .
\end{aligned}
$$

Notice that if we increase the value of $s$, the state space in which the controllability result holds has to take into account the fact that the value (or the normal derivative) of the function vanishes on $\partial \Omega \backslash \Gamma_{0}$. To state a result of this kind, we limit ourselves to the situation when $\Gamma_{0}$ is a side, e.g.

$$
\Gamma_{0}=\{0\} \times\left(0, l_{2}\right) \times \cdots \times\left(0, l_{n}\right) .
$$

Introduce the domain $\tilde{\Omega}=\left(-1, l_{1}\right) \times\left(0, l_{2}\right) \cdots \times\left(0, l_{n}\right)$ and a function $a \in C_{0}^{\infty}(\tilde{\Omega} \backslash \bar{\Omega})$, and consider the internal control problem

$$
i u_{t}+\Delta u+N(u)=i a(x) h(x, t), \quad x \in \tilde{\Omega}, t \in(0, T) .
$$

Taking the restriction to $\Omega \times(0, T)$ of solutions of $(79)$, we obtain as a corollary of Theorem 3.1 that both systems (69)-(70) and (69)-(71) are locally exactly controllable in some subspace of $H^{s}(\Omega)$ for any $s>s_{\alpha, n}$.

Corollary 3.15 For given $\alpha \geq 1, n \geq 2, \lambda \in \mathbb{R}, s>s_{\alpha, n}$ and $T>0$, there exists a constant $\delta>0$ such that for any $u_{0}, u_{1} \in H^{s}(\Omega)$ satisfying

$$
\left\|u_{i}\right\|_{H^{s}(\Omega)} \leq \delta, i=0,1
$$

and

$$
\begin{array}{cc}
u_{i}=\Delta u_{i}=\cdots=\Delta^{p} u_{i}=0 \quad x \in \partial \Omega \backslash \Gamma_{0}, p \leq\left[\frac{2 s-1}{4}\right], i=0,1 \\
\text { (resp. } \left.\quad \frac{\partial u_{i}}{\partial \nu}=\frac{\partial \Delta u_{i}}{\partial \nu}=\cdots=\frac{\partial \Delta^{p} u_{i}}{\partial \nu}=0 \quad x \in \partial \Omega \backslash \Gamma_{0}, p \leq\left[\frac{2 s-3}{4}\right], i=0,1\right),
\end{array}
$$

then one can choose a control input $h$ such that system (69)-(70) (resp. system (69)-(77)) admits a solution $u \in C\left([0, T] ; H^{s}(\Omega)\right)$ with

$$
u(x, 0)=u_{0}(x), \quad u(x, T)=u_{1}(x) .
$$

Remark 3.16 By using the same extension and restriction argument, one can derive a local controllability result in the space $H^{s}(\Omega)$ when $s>s_{\alpha, n}$ and for any given bounded smooth set $\Omega$, provided that the control is applied on the whole boundary (i.e. $\Gamma_{0}=\partial \Omega$ ). A result of this kind for which the critical Sobolev exponent $s=s_{c}=s_{2,2}=0$ is reached, is given in [4].

\section{Stabilization}

In this section we focus on the internal stabilization of the semilinear Schrödinger equation on the torus $\mathbb{T}^{n}$

$$
i u_{t}+\Delta u+N(u)=-i a^{2}(x) u, \quad x \in \mathbb{T}^{n}
$$

where $a$ is any smooth real function with $a \not \equiv 0$.

We have the following local exponential stability result which does not require the Geometric Control Condition.

Theorem 4.1 Let $a \in C_{0}^{\infty}\left(\mathbb{T}^{n}\right), a \not \equiv 0$, and let $s>s_{\alpha, N}$. Then there exist some constants $\nu, C$ such that every solution $u$ of $(80)$ issued from the initial state $u_{0} \in H^{s}\left(\mathbb{T}^{n}\right)$ satisfies

$$
\|u(t)\|_{s} \leq C e^{-\nu t}\left\|u_{0}\right\|_{s} \quad \forall t \geq 0 .
$$

Proof. We proceed as in 46 . The operator $A_{a}=i \Delta-a^{2}$ with domain $\mathcal{D}\left(A_{a}\right)=H^{s+2}\left(\mathbb{T}^{n}\right)$ generates a continuous group $\left(W_{a}(t)\right)_{t \in \mathbb{R}}$ of operators on $H^{s}\left(\mathbb{T}^{n}\right)$. The first step is to check that the semigroup $\left(W_{a}(t)\right)_{t \in \mathbb{R}^{+}}$is exponentially stable in $H^{s}\left(\mathbb{T}^{n}\right)$. This is done in the following 
Proposition 4.2 There exist positive constants $C>0$ and $\nu>0$ such that

$$
\left\|W_{a}(t) u_{0}\right\|_{s} \leq C e^{-\nu t}\left\|u_{0}\right\|_{s} \quad \forall t \geq 0
$$

Proof. When $s=0$, the exponential stability of $\left(W_{a}(t)\right)_{t \in \mathbb{R}^{+}}$is a direct consequence of Theorem 2.2, according to [36]. To prove (32) when $s=2$, we pick any $u_{0} \in H^{2}\left(\mathbb{T}^{n}\right)$ and set $v:=u_{t}$. Then $v$ solves the system

$$
\left\{\begin{array}{l}
v_{t}=i \Delta v-a^{2}(x) v, \quad x \in \mathbb{T}^{n} \\
v(x, 0)=v_{0}(x):=i \Delta u_{0}(x)-a^{2}(x) u_{0}(x) .
\end{array}\right.
$$

By the property 82 ) established when $s=0$, we have

$$
\|u(t)\|_{0} \leq C e^{-\nu t}\left\|u_{0}\right\|_{0}, \quad\|v(t)\|_{0} \leq C e^{-\nu t}\left\|v_{0}\right\|_{0}
$$

Since $i \Delta u=v+a^{2} u$, we conclude that

$$
\|u(t)\|_{2} \leq C e^{-\nu t}\left\|u_{0}\right\|_{2} \quad \forall t \geq 0
$$

An easy induction yields (82) for any $s \in 2 \mathbb{N}$. The proposition then follows by a classical interpolation argument.

Let us now turn our attention to the stability properties of the nonlinear system

$$
u_{t}=A_{a} u+i N(u), \quad u(., 0)=u_{0}
$$

that we shall write in its integral form

$$
u(t)=W_{a}(t) u_{0}+i \int_{0}^{t} W_{a}(t-\tau) N(u)(\tau) d \tau .
$$

At this point, we need to establish linear estimates when $W_{a}$ is substituted to $W$.

Lemma 4.3 Let $T>0, s \geq 0$ and $0 \leq b \leq 1$ be given. Then there exists a constant $C>0$ depending only on $T, s$ and $b$ such that

$$
\left\|W_{a}(t) \phi\right\|_{X_{s, b}^{T}} \leq C\|\phi\|_{s}
$$

for any $\phi \in H^{s}\left(\mathbb{T}^{n}\right)$

Proof. An application of Duhamel formula gives

$$
W_{a}(t) \phi=W(t) \phi-\int_{0}^{t} W(t-\tau)\left(a^{2} W_{a}(\tau) \phi\right) d \tau .
$$

It follows that

$$
\begin{aligned}
\left\|W_{a}(t) \phi\right\|_{X_{s, b}^{T}} & \leq\|W(t) \phi\|_{X_{s, b}^{T}}+\left\|\int_{0}^{t} W(t-\tau)\left(a^{2} W_{a}(\tau) \phi\right) d \tau\right\|_{X_{s, b}^{T}} \\
& \leq C\|\phi\|_{s}+C\left\|a^{2} W_{a}(t) \phi\right\|_{X_{s, b-1}^{T}} \\
& \leq C\|\phi\|_{s}+C\left\|W_{a}(t) \phi\right\|_{L^{2}\left(0, T ; H^{s}\left(\mathbb{T}^{n}\right)\right)} \quad(\text { as } b-1 \leq 0) \\
& \leq C\|\phi\|_{s},
\end{aligned}
$$

as desired.

Lemma 4.4 Let $T>0, s \geq 0$, and $b \in\left(\frac{1}{2}, 1\right)$ be given. Then there exists a constant $C>0$ depending only on $T, s$ and $b$ such that

$$
\left\|\int_{0}^{t} W_{a}(t-\tau) f(\tau) d \tau\right\|_{X_{s, b}^{T}} \leq C\|f\|_{X_{s, b-1}^{T}}
$$

for any $f \in X_{s, b-1}^{T}$. 
Proof. It follows from (85) that

$$
\int_{0}^{t} W_{a}(t-\tau) f(\tau) d \tau=\int_{0}^{t} W(t-\tau) f(\tau) d \tau-\int_{0}^{t} W(t-\tau) a^{2}\left(\int_{0}^{\tau} W_{a}(\tau-s) f(s) d s\right) d \tau
$$

hence

$$
\begin{aligned}
\left\|\int_{0}^{t} W_{a}(t-\tau) f(\tau) d \tau\right\|_{X_{s, b}^{T}} & \leq C\|f\|_{X_{s, b-1}^{T}}+C\left\|a^{2} \int_{0}^{t} W_{a}(t-s) f(s) d s\right\|_{X_{s, b-1}^{T}} \\
& \leq C\|f\|_{X_{s, b-1}^{T}}+C\left\|\int_{0}^{t} W_{a}(t-s) f(s) d s\right\|_{X_{s, 0}^{T}} \\
& \leq C\|f\|_{X_{s, b-1}^{T}}+C T^{\alpha}\left\|\int_{0}^{t} W_{a}(t-s) f(s) d s\right\|_{X_{s, b}^{T}}
\end{aligned}
$$

for some constant $\alpha>0$, by virtue of Lemmas 3.2 and [50, Lemma 2.11]. The result follows at once if $T$ is small enough, say $T<T_{0}$. For $T \geq T_{0}$, the result follows from Lemma 4.3 and an easy induction.

Let us now proceed to the proof of the exponential stability of the system (80). Pick a number $s \geq 0$. According to Proposition 4.2, there exist positive constants $C, \nu$ such that

$$
\left\|W_{a}(t) u_{0}\right\|_{s} \leq C e^{-\nu t}\left\|u_{0}\right\|_{s} \quad \forall t \geq 0 .
$$

Pick a time $T>0$ such that

$$
C e^{-\nu T}<\frac{1}{4}
$$

and fix a number $b \in\left(\frac{1}{2}, 1\right)$. We seek a solution $u$ of the integral equation (84) in the form of a fixed point of the map

$$
\Gamma(u)=W_{a}(t) u_{0}+i \int_{0}^{t} W_{a}(t-\tau) N(u)(\tau) d \tau
$$

in some ball $B_{M}$ of the space $X_{s, b}^{T}$. This will be done provided that $\left\|u_{0}\right\|_{s} \leq \delta$ where $\delta$ is a small number to be determined. Furthermore, to ensure the exponential stability, $\delta$ and $M$ will be chosen in such a way that $\|u(T)\|_{s} \leq\left\|u_{0}\right\|_{s} / 2$. Pick for the moment any $\delta>0$ and $M>0$, and let $u_{0} \in H^{s}\left(\mathbb{T}^{n}\right)$ be such that $\left\|u_{0}\right\|_{s} \leq \delta$. By computations similar to those displayed in the proof of Theorem 3.1 with $W_{a}(t)$ substituted to $W(t)$, we arrive to

$$
\|\Gamma(u)\|_{X_{s, b}^{T}} \leq c|| u_{0} \|_{s}+c M^{\alpha+1} \quad \forall u \in B_{M}
$$

and

$$
\|\Gamma(u)-\Gamma(v)\|_{X_{s, b}^{T}} \leq c M^{\alpha}\|u-v\|_{X_{s, b}^{T}} \quad \forall u, v \in B_{M}
$$

for some constant $c>0$ independent of $\delta, M$, and $u_{0}$. On the other hand, using the estimate of $\|\omega(T, u)\|_{s}$ in the proof of Theorem 3.1, we obtain

$$
\begin{aligned}
\|\Gamma(u)(T)\|_{s} & \leq\left\|W_{a}(T) u_{0}\right\|_{s}+\left\|\int_{0}^{T} W_{a}(T-t) N(u)(t) d t\right\|_{s} \\
& \leq \frac{1}{4}\left\|u_{0}\right\|_{s}+c M^{\alpha+1} .
\end{aligned}
$$

Pick $\delta=4 c M^{\alpha+1}$ where $M>0$ is chosen so that

$$
\left(4 c^{2}+c\right) M^{\alpha+1} \leq M \text { and } c M^{\alpha} \leq \frac{1}{2} .
$$

Then we have

$$
\begin{aligned}
\|\Gamma(u)\|_{X_{s, b}^{T}} & \leq M \quad \forall u \in B_{M} \\
\|\Gamma(u)-\Gamma(v)\|_{X_{s, b}^{T}} & \leq \frac{1}{2}\|u-v\|_{X_{s, b}^{T}} \quad \forall u, v \in B_{M} .
\end{aligned}
$$

Thus the map $\Gamma$, which is a contraction in $B_{M}$, has a fixed point $u \in B_{M}$. By construction, $u$ fulfills

$$
\|u(T)\|_{s}=\|\Gamma(u)(T)\|_{s} \leq \frac{\delta}{2} .
$$


Assume now that $0<\left\|u_{0}\right\|_{s}<\delta$. Changing $\delta$ into $\delta^{\prime}:=\left\|u_{0}\right\|_{s}$ and $M$ into $M^{\prime}:=\left(\delta^{\prime} / \delta\right)^{\frac{1}{\alpha+1}} M$, we obtain that $\|u(T)\|_{s} \leq\left\|u_{0}\right\|_{s} / 2$, and an obvious induction yields $\|u(k T)\|_{s} \leq 2^{-k}\left\|u_{0}\right\|_{s}$ for any $k \geq 0$. As $X_{s, b}^{T} \subset C\left([0, T] ; H^{s}\left(\mathbb{T}^{n}\right)\right)$ for $b>1 / 2$, and $\|u\|_{X_{s, b}^{T}} \leq M=(\delta /(4 c))^{\frac{1}{\alpha+1}}$, we infer by the semigroup property that there exist some constants $C^{\prime}>0, \nu^{\prime}>0$ such that

$$
\|u(t)\|_{s} \leq C^{\prime} e^{-\nu^{\prime} t}\left\|u_{0}\right\|_{s}
$$

The proof is complete.

\section{Appendix}

\subsection{Proof of Proposition 3.4.}

We proceed as in [9, pp. 115-118]. We first introduce some notations. Let $|x|_{\infty}:=\sup _{1 \leq i \leq n}\left|x_{i}\right|$ for $x=\left(x_{i}\right)_{1 \leq i \leq n} \in \mathbb{R}^{n}$. We introduce a dyadic partition of $\mathbb{R}^{n}$

$$
\mathbb{Z}^{n}=\cup_{j \geq 0} D_{j}
$$

where $D_{0}=\{0\}$, and $D_{j}=\left\{k \in \mathbb{Z}^{n} ; 2^{j-1} \leq|k|_{\infty}<2^{j}\right\}$ for $j \geq 1$. For any Hölder exponent $p, q \in[1,+\infty]$, we write $L_{t}^{p} L_{x}^{q}$ for $L^{p}\left(\mathbb{R}_{t}, L^{q}\left(\mathbb{T}_{x}^{n}\right)\right)$. The (discrete) cube of center $x_{0} \in \mathbb{R}^{n}$ and sidelength $2 R>0$ is

$$
Q\left(x_{0}, R\right)=\left\{k \in \mathbb{Z}^{n} ;\left|k-x_{0}\right|_{\infty} \leq R\right\} .
$$

The Strichartz estimate $([\mathbb{8},[16])$

$$
\|u\|_{L_{t}^{4} L_{x}^{4}} \leq c\|u\|_{X_{s, b}}, \quad s>\frac{n}{2}-\frac{n+2}{4}, b>\frac{1}{2},
$$

when combined with the standard estimates

$$
\begin{aligned}
\|u\|_{L_{t}^{\infty} L_{x}^{2}} & \leq c\|u\|_{X_{0, b}}, \quad b>\frac{1}{2} \\
\|u\|_{L_{t}^{2} L_{x}^{2}} & =\|u\|_{X_{0,0}}
\end{aligned}
$$

and Sobolev embedding theorem, gives by interpolation the following result.

Lemma 5.1 (10, cor. 2.2]) Let $n \geq 2$.

(i) For all $p, q, s$ satisfying

$$
0<\frac{1}{p} \leq \frac{1}{4}, 0<\frac{1}{q} \leq \frac{1}{2}-\frac{1}{p}, s>\frac{n}{2}-\frac{2}{p}-\frac{n}{q}
$$

there exists a number $b \in\left(0, \frac{1}{2}\right)$ such that for all $u \in X_{s, b}$, it holds

$$
\|u\|_{L_{t}^{p} L_{x}^{q}} \leq c\|u\|_{X_{s, b}}
$$

(ii) For all $p, q, s, b$ satisfying

$$
0 \leq \frac{1}{p} \leq \frac{1}{q} \leq \frac{1}{2} \leq \frac{1}{p}+\frac{1}{q} \leq 1, s>(n-2)\left(\frac{1}{2}-\frac{1}{q}\right), \text { and } b>1-\frac{1}{p}-\frac{1}{q}
$$

then for all $u \in X_{s, b}$, (87) holds.

Let $\mathcal{F}_{x}$ denote the Fourier transform in $x$, and let $1_{Q}$ denote the characteristic function of the cube $Q$. The following result, inspired by an observation made in [8], indicates that for a function spatially supported in a cube, only the sidelength of the cube (not its center) comes into play in (87).

Lemma 5.2 (10, Lemma 2.4]) Assume that for $p, q, s, b$ the estimate (87) is valid. Then there exists a constant $c>0$ such that for any cube $Q$ of center $x_{0} \in \mathbb{R}^{n}$ and sidelength $R>0$ it holds

$$
\left\|\left(\mathcal{F}_{x}^{-1} 1_{Q} \mathcal{F}_{x}\right) u\right\|_{L_{t}^{p} L_{x}^{q}} \leq c R^{s}\|u\|_{X_{0, b}} .
$$


It follows that if (86) (or (88)) holds and if $u=u(x, t)$ is a function decomposed as

$$
u(x, t)=\sum_{\left|k-x_{0}\right|_{\infty} \leq R} \int_{\mathbb{R}} \hat{u}(k, \tau) e^{i(k \cdot x+\tau t)} d \tau
$$

then

$$
\|u\|_{L_{t}^{p} L_{x}^{q}} \leq c R^{s}\|u\|_{X_{0, b}}=c R^{s}\left(\sum_{\left|k-x_{0}\right|_{\infty} \leq R} \int_{\mathbb{R}}\left\langle\tau+|k|^{2}\right\rangle^{2 b}|\hat{u}(k, \tau)|^{2} d \tau\right)^{\frac{1}{2}} .
$$

Let the functions $u_{1}, \ldots, u_{\alpha+1} \in X_{s, b}$ be given, where $s$ and $b$ denote some positive numbers, and let us set

$$
u=\tilde{u}_{1} \tilde{u}_{2} \cdots \tilde{u}_{\alpha+1}
$$

where $\tilde{u}_{i}$ is $u_{i}$ or $\overline{u_{i}}$. To estimate $\|u\|_{X_{s,-b}}$ we proceed by duality, estimating the integral $\int_{\mathbb{R}} \int_{\mathbb{T}^{n}} u \bar{v} d x d t$ for any $v \in X_{-s, b}$ with $\|v\|_{X_{-s, b}} \leq 1$. By Plancherel theorem

$$
\begin{aligned}
\int_{\mathbb{R}} \int_{\mathbb{T}^{n}} u \bar{v} d x d t & =\sum_{k \in \mathbb{Z}^{n}} \int_{\mathbb{R}} \hat{u}(k, \tau) \overline{\hat{v}}(k, \tau) d \tau \\
& =\sum_{k_{1} \cdots k_{\alpha+1}} \int_{\tau_{1} \cdots \tau_{\alpha+1}}\langle k\rangle^{s}\left(\prod_{i=1}^{\alpha+1} \hat{\tilde{u}}_{i}\left(k_{i}, \tau_{i}\right)\right)\langle k\rangle^{-s} \overline{\hat{v}}(k, \tau)
\end{aligned}
$$

where $k=k_{1}+\cdots+k_{\alpha+1}$ and $\tau=\tau_{1}+\cdots+\tau_{\alpha+1}$. Notice that $\hat{\bar{u}}\left(k_{i}, \tau_{i}\right)=\overline{\hat{u}_{i}\left(-k_{i},-\tau_{i}\right)}$. Writing $k_{i} \in D_{j_{i}}$, $j_{i} \geq 0$, we obtain

$$
\left|\int_{\mathbb{R}} \int_{\mathbb{T}^{n}} u \bar{v} d x d t\right| \leq \sum_{j_{1} \cdots j_{\alpha+1}} \sum_{k_{i} \in D_{j_{i}}} \int_{\tau_{1} \cdots \tau_{\alpha+1}}\langle k\rangle^{s}\left(\prod_{i=1}^{\alpha+1}\left|\hat{u}_{i}\left(k_{i}, \tau_{i}\right)\right|\right)\langle k\rangle^{-s}|\hat{v}(k, \tau)|,
$$

where now $k= \pm k_{1} \cdots \pm k_{\alpha+1}, \tau= \pm \tau_{1} \cdots \pm \tau_{\alpha+1}\left(+k_{i}\right.$ if $\tilde{u}_{i}=u_{i},-k_{i}$ if $\tilde{u}_{i}=\overline{u_{i}}$, and the same for $\left.\pm \tau_{i}\right)$. We shall focus on the sum $\Sigma=\sum_{j_{1} \geq j_{2} \geq \cdots \geq j_{\alpha+1}}$, the other contributions leading to similar bounds. As $\left|k_{i}\right|_{\infty} \leq 2\left|k_{1}\right|_{\infty}$ for $i \geq 2$, we have that

$$
\Sigma \leq c \sum_{j_{1} \geq \cdots \geq j_{\alpha+1}} 2^{j_{1} s} \sum_{k_{i} \in D_{j_{i}}} \int_{\tau_{1} \cdots \tau_{\alpha+1}}\left(\prod_{i=1}^{\alpha+1}\left|\hat{u}_{i}\left(k_{i}, \tau_{i}\right)\right|\right)\langle k\rangle^{-s}|\hat{v}(k, \tau)| .
$$

Pick $\gamma \in \mathbb{N}^{*}$ with

$$
\alpha \leq 2^{\gamma-2}
$$

and split $\Sigma$ into $\Sigma_{1}+\Sigma_{2}$ where $\Sigma_{1}$ corresponds to the $j_{1}, \ldots, j_{\alpha+1}$ for which

$$
j_{1} \geq j_{2}+\gamma+2 \geq j_{2} \geq j_{3} \geq \cdots \geq j_{\alpha+1}
$$

Consider a "partition" of $D_{j_{1}}$ into a collection of cubes $Q_{l}$ of sidelength $2^{j_{2}}$

$$
D_{j_{1}}=\cup_{l} Q_{l}
$$

Note that each $k \in D_{j_{1}}$ belongs to at most $2^{n}$ cubes $Q_{l}$. For any $l$, we denote by $\tilde{Q}_{l}$ the cube of sidelength $2^{j_{2}+\gamma}$ with the same center as $Q_{l}$ if $k=k_{1} \pm k_{2} \cdots$, and with center the opposite of that of $Q_{l}$ if $k=$ $-k_{1} \pm k_{2} \cdots$. We claim that $k \in \tilde{Q}_{l}$ when $k_{1} \in Q_{l}$ and $k_{i} \in D_{j_{i}}$ for $i \geq 2$. Indeed

$$
\left|k_{2}\right|_{\infty}+\cdots+\left|k_{\alpha+1}\right|_{\infty} \leq \alpha 2^{j_{2}} \leq 2^{j_{2}+\gamma-2},
$$

hence if $Q_{l}=Q\left(x_{0}, 2^{j_{2}-1}\right)$

$$
\left| \pm x_{0}-k\right|_{\infty} \leq\left| \pm x_{0}- \pm k_{1}\right|_{\infty}+\left|k_{2}\right|_{\infty}+\cdots+\left|k_{\alpha+1}\right|_{\infty} \leq 2^{j_{2}-1}+2^{j_{2}+\gamma-2} \leq 2^{j_{2}+\gamma-1} .
$$


Notice also that $\tilde{Q}_{l} \subset D_{j_{1}-1} \cup D_{j_{1}} \cup D_{j_{1}+1}$ since the sidelength of $\tilde{Q}_{l}$ is at most $2^{j_{1}-2}$ and $Q_{l} \subset D_{j_{1}}$. It follows that

$$
\Sigma_{1} \leq c \sum_{\substack{j_{1} \geq j_{2}+\gamma+2 \\ j_{2} \geq j_{3} \geq \cdots \geq j_{\alpha+1}}} 2^{j_{1} s} \sum_{l} \sum_{\substack{k_{1} \in Q_{l} \\ k_{l}}} \sum_{\substack{k_{2} \in D_{j_{2}}, k_{\alpha+1} \in D_{j_{\alpha+1}}}} \int_{\tau_{1} \cdots \tau_{\alpha+1}}\left(\prod_{i=1}^{\alpha+1}\left|\hat{u}_{i}\left(k_{i}, \tau_{i}\right)\right|\right) 1_{\tilde{Q}_{l}}(k)\langle k\rangle^{-s}|\hat{v}(k, \tau)| .
$$

Let us introduce the functions

$$
\begin{aligned}
f_{l}(x, t) & =\sum_{k \in Q_{l}} \int_{\mathbb{R}}\left|\hat{u}_{1}(k, \tau)\right| e^{i(k \cdot x+\tau t)} d \tau \\
g_{l}(x, t) & =\sum_{k \in \tilde{Q}_{l}} \int_{\mathbb{R}}\langle k\rangle^{-s}|\hat{v}(k, \tau)| e^{i(k \cdot x+\tau t)} d \tau
\end{aligned}
$$

and

$$
h_{i}(x, t)=\sum_{k \in D_{j_{i}}} \int_{\mathbb{R}}\left|\hat{u}_{i}(k, \tau)\right| e^{i(k \cdot x+\tau t)} d \tau \quad \text { for } i=2, \ldots, \alpha+1 .
$$

By Plancherel theorem

$$
\Sigma_{1} \leq c \sum_{\substack{j_{1} \geq j_{2}+\gamma+2 \\ j_{2} \geq j_{3} \geq \cdots \geq j_{\alpha+1}}} 2^{j_{1} s} \sum_{l} \int_{\mathbb{R}} \int_{\mathbb{T}^{n}}\left|f_{l} h_{2} \cdots h_{\alpha+1} g_{l}\right| d x d t
$$

Pick Hölder exponents $p_{1}, q_{1}, p_{2}, q_{2} \in[1, \infty)$ such that

$$
\begin{aligned}
& \frac{3}{p_{1}}+\frac{\alpha-1}{p_{2}}=1 \\
& \frac{3}{q_{1}}+\frac{\alpha-1}{q_{2}}=1
\end{aligned}
$$

We have that

$$
\int_{\mathbb{R}} \int_{\mathbb{T}^{n}}\left|f_{l} h_{2} \cdots h_{\alpha+1} g_{l}\right| d x d t \leq\left\|f_{l}\right\|_{L_{t}^{p_{1}} L_{x}^{q_{1}}}\left\|g_{l}\right\|_{L_{t}^{p_{1}} L_{x}^{q_{1}}}\left\|h_{2}\right\|_{L_{t}^{p_{1}} L_{x}^{q_{1}}} \prod_{i=3}^{\alpha+1}\left\|h_{i}\right\|_{L_{t}^{p_{2}} L_{x}^{q_{2}}}
$$

Assume that for some exponents $s_{1}, b_{1}, s_{2}, b_{2}$ the following estimates hold

$$
\begin{aligned}
& \|u\|_{L_{t}^{p_{1}} L_{x}^{q_{1}}} \leq c\|u\|_{X_{s_{1}, b_{1}}}, \\
& \|u\|_{L_{t}^{p_{2}} L_{x}^{q_{2}}} \leq c\|u\|_{X_{s_{2}, b_{2}}} \text {. }
\end{aligned}
$$

Then, by (90) and the fact that the sidelength of $Q_{l}$ (resp. $\tilde{Q}_{l}$ ) is $2^{j_{2}}$ (resp. $2^{j_{2}+\gamma}$ ), we have

$$
\begin{aligned}
\left\|f_{l}\right\|_{L_{t}^{p_{1}} L_{x}^{q_{1}}} & \leq c 2^{j_{2} s_{1}}\left(\sum_{k \in Q_{l}} \int_{\tau}\left\langle\tau+|k|^{2}\right\rangle^{2 b_{1}}\left|\hat{u}_{1}\right|^{2}\right)^{\frac{1}{2}} \\
\left\|g_{l}\right\|_{L_{t}^{p_{1}} L_{x}^{q_{1}}} & \leq c 2^{j_{2} s_{1}}\left(\sum_{k \in \tilde{Q}_{l}} \int_{\tau}\left\langle\tau+|k|^{2}\right\rangle^{2 b_{1}}\langle k\rangle^{-2 s}|\hat{v}|^{2}\right)^{\frac{1}{2}} \\
\left\|h_{2}\right\|_{L_{t}^{p_{1}} L_{x}^{q_{1}}} & \leq c 2^{j_{2} s_{1}}\left(\sum_{k \in D_{j_{2}}} \int_{\tau}\left\langle\tau+|k|^{2}\right\rangle^{2 b_{1}}\left|\hat{u}_{2}\right|^{2}\right)^{\frac{1}{2}}
\end{aligned}
$$

and for $i=3, \ldots, \alpha+1$

$$
\begin{aligned}
\left\|h_{i}\right\|_{L_{t}^{p_{2}} L_{x}^{q_{2}}} & \leq c 2^{j_{i} s_{2}}\left(\sum_{k \in D_{j_{i}}} \int_{\tau}\left\langle\tau+|k|^{2}\right\rangle^{2 b_{2}}\left|\hat{u}_{i}\right|^{2}\right)^{\frac{1}{2}} \\
& \leq c\left(\sum_{k \in D_{j_{i}}} \int_{\tau}\left\langle\tau+|k|^{2}\right\rangle^{2 b_{2}}\langle k\rangle^{2 s_{2}}\left|\hat{u}_{i}\right|^{2}\right)^{\frac{1}{2}}
\end{aligned}
$$


Using Cauchy-Schwarz in $\sum_{l}$, we obtain

$$
\begin{aligned}
\Sigma_{1} \leq & c \sum_{\substack{j_{1} \geq j_{2}+\gamma+2 \\
j_{2} \geq j_{3} \geq \cdots \geq j_{\alpha+1}}} 2^{j_{1} s+3 j_{2} s_{1}}\left(\sum_{l} \sum_{k \in Q_{l}} \int_{\tau}\left\langle\tau+|k|^{2}\right\rangle^{2 b_{1}}\left|\hat{u}_{1}\right|^{2}\right)^{\frac{1}{2}}\left(\sum_{l} \sum_{k \in \tilde{Q}_{l}} \int_{\tau}\left\langle\tau+|k|^{2}\right\rangle^{2 b_{1}}\langle k\rangle^{-2 s}|\hat{v}|^{2}\right)^{\frac{1}{2}} \\
& \left(\sum_{k \in D_{j_{2}}} \int_{\tau}\left\langle\tau+|k|^{2}\right\rangle^{2 b_{1}}\left|\hat{u}_{2}\right|^{2}\right)^{\frac{1}{2}} \prod_{i=3}^{\alpha+1}\left(\sum_{k \in D_{j_{i}}} \int_{\tau}\left\langle\tau+|k|^{2}\right\rangle^{2 b_{2}}\langle k\rangle^{2 s_{2}}\left|\hat{u}_{i}\right|^{2}\right)^{\frac{1}{2}} \\
\leq & c \sum_{\substack{j_{1} \geq j_{2}+\gamma+2 \\
j_{2} \geq j_{3} \geq \cdots \geq j_{\alpha+1}}}\left(\sum_{k \in D_{j_{1}}} \int_{\tau}\left\langle\tau+|k|^{2}\right\rangle^{2 b_{1}}\langle k\rangle^{2 s}\left|\hat{u}_{1}\right|^{2}\right)^{\frac{1}{2}}\left(\sum_{k \in D_{j_{1}-1} \cup D_{j_{1}} \cup D_{j_{1}+1}}\left\langle\tau+|k|^{2}\right\rangle^{2 b_{1}}\langle k\rangle^{-2 s}|\hat{v}|^{2}\right)^{\frac{1}{2}} \\
& \left(\sum_{k \in D_{j_{2}}} \int_{\tau}\left\langle\tau+|k|^{2}\right\rangle^{2 b_{1}}\langle k\rangle^{6 s_{1}}\left|\hat{u}_{2}\right|^{2}\right)^{\frac{1}{2}} \prod_{i=3}^{\alpha+1}\left(\sum_{k \in D_{j_{i}}} \int_{\tau}\left\langle\tau+|k|^{2}\right\rangle^{2 b_{2}}\langle k\rangle^{2 s_{2}}\left|\hat{u}_{i}\right|^{2}\right)^{\frac{1}{2}}
\end{aligned}
$$

We used the fact that a point $k \in D_{j_{1}-1} \cup D_{j_{1}} \cup D_{j_{1}+1}$ belongs to (at most) a finite number of cubes $\tilde{Q}_{l}$, bounded by $\left(2^{\gamma+2}+1\right)^{n}$. A sum $\sum_{j_{i} \geq 0}\left(\sum_{k \in D_{j_{i}}} \int_{\tau}\left\langle\tau+|k|^{2}\right\rangle^{2 b_{2}}\langle k\rangle^{2 s_{2}}\left|\hat{u}_{i}\right|^{2}\right)^{\frac{1}{2}}$ can be estimated by $c|| u_{i} \|_{X_{s_{2}+\varepsilon, b_{2}}}$ for any $\varepsilon>0$ thanks to Cauchy-Schwarz. Summing successively in $k_{\alpha+1}, \ldots, k_{1}$, we arrive at

$$
\Sigma_{1} \leq c|| u_{1}\left\|_{X_{s, b_{1}}}\right\| v\left\|_{X_{-s, b_{1}}}\right\| u_{2}\left\|_{X_{3_{1}+\varepsilon, b_{1}}} \prod_{i=3}^{\alpha+1}\right\| u_{i} \|_{X_{s_{2}+\varepsilon, b_{2}}}
$$

The same bound for $\Sigma_{2}$ can be obtained by a more simple analysis. Indeed, as $j_{1} \leq j_{2}+\gamma+1$ in the sum over $j_{1}, \ldots, j_{\alpha+1}$, we obtain

$$
\Sigma_{2} \leq c \sum_{\substack{j_{1} \leq j_{2}+\gamma+1 \\ j_{2} \geq j_{3} \geq \cdots \geq j_{\alpha+1}}} 2^{j_{1} s} \int_{\mathbb{R}} \int_{\mathbb{T}^{n}}\left|f h_{2} \cdots h_{\alpha+1} g\right| d x d t
$$

where

$$
\begin{aligned}
f(x, t) & =\sum_{k \in D_{j_{1}}} \int_{\mathbb{R}}\left|\hat{u}_{1}(k, \tau)\right| e^{i(k \cdot x+\tau t)} d \tau \\
g(x, t) & =\sum_{|k| \leq\left(2^{\gamma+1}+\alpha\right) 2^{j_{2}}} \int_{\mathbb{R}}\langle k\rangle^{-s}|\hat{v}(k, \tau)| e^{i(k \cdot x+\tau t)} d \tau
\end{aligned}
$$

and $h_{2}, \ldots, h_{\alpha+1}$ as above. Since $2^{j_{1} s_{1}} \leq c 2^{j_{2} s_{1}}$, we still have

$$
\begin{aligned}
\|f\|_{L_{t}^{p_{1}} L_{x}^{q_{1}}} & \leq c 2^{j_{2} s_{1}}\left(\sum_{k \in D_{j_{1}}} \int_{\tau}\left\langle\tau+|k|^{2}\right\rangle^{2 b_{1}}\left|\hat{u}_{1}\right|^{2}\right)^{\frac{1}{2}} \\
\|g\|_{L_{t}^{p_{1}} L_{x}^{q_{1}}} & \leq c 2^{j_{2} s_{1}}\left(\sum_{k \in \mathbb{Z}^{n}} \int_{\tau}\left\langle\tau+|k|^{2}\right\rangle^{2 b_{1}}\langle k\rangle^{-2 s}|\hat{v}|^{2}\right)^{\frac{1}{2}}
\end{aligned}
$$

Next, $\Sigma_{2}$ is estimated as $\Sigma_{1}$ (see above). At this stage, we have proved that

$$
\Sigma \leq c\left\|u_{1}\right\|_{X_{s, b_{1}}}\|v\|_{X_{-s, b_{1}}}\left\|u_{2}\right\|_{X_{3_{1}+\varepsilon, b_{1}}} \prod_{i=3}^{\alpha+1}\left\|u_{i}\right\|_{X_{s_{2}+\varepsilon}, b_{2}}
$$

where $\varepsilon>0$ is arbitrary small, the exponents $s_{1}, b_{1}, s_{2}, b_{2}$ are taken so that (94)-(95) are satisfied, with the Hölder exponents $p_{1}, q_{1}, p_{2}, q_{2}$ satisfying (92)-(93). The proof will be complete if, in addition, we have

$$
s \geq \sup \left\{3 s_{1}+\varepsilon, s_{2}+\varepsilon\right\}, \quad b_{1}<\frac{1}{2}, b_{2}<\frac{1}{2} .
$$


We distinguish three cases: (i) $\alpha \geq 3$; (ii) $\alpha=2$; (iii) $\alpha=1$.

(i) $\alpha \geq 3$

We aim to reach any value $s>s_{c}$. To find the sets of exponents $\left(p_{1}, q_{1}, s_{1}, b_{1}\right),\left(p_{2}, q_{2}, s_{2}, b_{2}\right)$ satisfying (86), (92) and (93), and leading to the "smallest" value of $s$, we are let to minimize the functional $\sup \left\{3 \sigma_{1}, \sigma_{2}\right\}$, where

$$
\begin{aligned}
\sigma_{1} & =\frac{n}{2}-\left(\frac{2}{p_{1}}+\frac{n}{q_{1}}\right) \\
\sigma_{2} & =\frac{n}{2}-\left(\frac{2}{p_{2}}+\frac{n}{q_{2}}\right)
\end{aligned}
$$

under the constraints

$$
\begin{aligned}
& 4 \leq p_{1}<\infty \\
& 0<\frac{1}{q_{1}} \leq \frac{1}{2}-\frac{1}{p_{1}} \\
& 4 \leq p_{2}<\infty \\
& 0<\frac{1}{q_{2}} \leq \frac{1}{2}-\frac{1}{p_{2}} \\
& \frac{3}{p_{1}}+\frac{\alpha-1}{p_{2}}=1 \\
& \frac{3}{q_{1}}+\frac{\alpha-1}{q_{2}}=1 .
\end{aligned}
$$

At this point, it is convenient to introduce the numbers $r_{1}, r_{2}$ with

$$
\begin{aligned}
\frac{1}{r_{1}} & =\frac{2}{p_{1}}+\frac{n}{q_{1}} \\
\frac{1}{r_{2}} & =\frac{2}{p_{2}}+\frac{n}{q_{2}} .
\end{aligned}
$$

Note that, by (107)-(108),

$$
\frac{3}{r_{1}}+\frac{\alpha-1}{r_{2}}=n+2 \text {. }
$$

Therefore, $3 \sigma_{1}=\frac{n}{2}-2+\frac{\alpha-1}{r_{2}}$ (resp. $\sigma_{2}=\frac{n}{2}-\frac{1}{r_{2}}$ ) is a nonincreasing function (resp. a nondecreasing function) of $r_{2}$. Thus the least value of $\sup \left\{3 \sigma_{1}, \sigma_{2}\right\}$ is achieved when $3 \sigma_{1}=\sigma_{2}$, which yields

$$
r_{2}=\frac{\alpha}{2}, r_{1}=3\left(n+\frac{2}{\alpha}\right)^{-1}, \quad 3 \sigma_{1}=\sigma_{2}=\frac{n}{2}-\frac{2}{\alpha} .
$$

It remains to find $p_{1}, q_{1}, p_{2}, q_{2}$ satisfying (103)-(110). Note first that (108) is satisfied whenever (107) is, by (111). Taking $p_{1}$ as variable, we infer from (107), (109) and (110) that

$$
\frac{1}{p_{2}}=\frac{1}{\alpha-1}\left(1-\frac{3}{p_{1}}\right), \quad \frac{1}{q_{1}}=\frac{1}{3}\left(1+\frac{2}{n \alpha}\right)-\frac{2}{n p_{1}}, \quad \frac{1}{q_{2}}=\frac{2}{n(\alpha-1)}\left(\frac{3}{p_{1}}-\frac{1}{\alpha}\right) .
$$

The constraints (105), (104) and (106) are found to be respectively equivalent to

$$
p_{1} \leq 3\left(1-\frac{\alpha-1}{4}\right)^{-1}(\text { for } \quad \alpha \leq 4), \quad p_{1} \geq \sup \left\{6\left(n+\frac{2}{\alpha}\right)^{-1}, 6\left(1-\frac{2}{n}\right)\left(1-\frac{4}{n \alpha}\right)^{-1}\right\}, \quad p_{1}<3 \alpha .
$$

The value $p_{1}=6$ fulfills all the requirements in 113 . Let now $s>\frac{n}{2}-\frac{2}{\alpha}$ be given. Choose $\varepsilon>0$ such that $4 \varepsilon<s-\left(\frac{n}{2}-\frac{2}{\alpha}\right)$, and pick $s_{1} \in\left(\sigma_{1}, \sigma_{1}+\varepsilon\right)$, and $s_{2} \in\left(\sigma_{2}, \sigma_{2}+\varepsilon\right)$. Then (94) and (95) hold for some numbers $b_{1}<\frac{1}{2}, b_{2}<\frac{1}{2}$, according to Lemma 5.1. Set finally $b=\sup \left\{b_{1}, b_{2}\right\}$. Then we have

$$
\Sigma \leq c\left(\prod_{i=1}^{\alpha+1}\left\|u_{i}\right\|_{X_{s, b}}\right)\|v\|_{X_{-s, b}}
$$

which gives (64).

(ii) $\alpha=2$ 
Observe first that the approach followed in (i) does not work for $n>2$. Indeed, the constraints (103)-(112) impose $p_{1}=p_{2}=q_{1}=q_{2}=4$, and the equation $3 \sigma_{1}=\sigma_{2}$ is then satisfied only for $n=2$. Assume $n \geq 3$. We now search a couple $\left(p_{1}, q_{1}\right)$ satisfying

$$
0<\frac{1}{p_{1}} \leq \frac{1}{q_{1}} \leq \frac{1}{2} \leq \frac{1}{p_{1}}+\frac{1}{q_{1}} \leq 1, \quad s_{1}>(n-2)\left(\frac{1}{2}-\frac{1}{q_{1}}\right), \quad b_{1}>1-\frac{1}{p_{1}}-\frac{1}{q_{1}}
$$

while $\left(p_{2}, q_{2}\right)$ still satisfies

$$
0<\frac{1}{p_{2}} \leq \frac{1}{4}, \quad 0 \leq \frac{1}{q_{2}} \leq \frac{1}{2}-\frac{1}{p_{2}}, \quad s_{2}>\frac{n}{2}-\frac{2}{p_{2}}-\frac{n}{q_{2}} .
$$

The Hölder exponents $\left(p_{1}, q_{1}\right)$ and $\left(p_{2}, q_{2}\right)$ have to satisfy the relations

$$
\begin{aligned}
& \frac{3}{p_{1}}+\frac{1}{p_{2}}=1, \\
& \frac{3}{q_{1}}+\frac{1}{q_{2}}=1 .
\end{aligned}
$$

We still minimize the functional $\sup \left\{3 \sigma_{1}, \sigma_{2}\right\}$, where

$$
\sigma_{1}=(n-2)\left(\frac{1}{2}-\frac{1}{q_{1}}\right), \quad \sigma_{2}=\frac{n}{2}-\frac{2}{p_{2}}-\frac{n}{q_{2}}=\frac{n}{2}-\frac{2}{p_{2}}-n\left(1-\frac{3}{q_{1}}\right)
$$

by solving in $q_{1}$ the equation $3 \sigma_{1}=\sigma_{2}$. Taking $p_{2}=4$ to produce the least value of $\sigma_{2}$, we find as solution $q_{1}=3\left(1+\frac{1}{4 n-5}\right) \in(3,4)$, which yields $p_{1}=4$ and $q_{2}=4(n-1)$ by $(116)-(117)$, and

$$
3 \sigma_{1}=\sigma_{2}=\frac{n}{2}-\frac{3}{4}-\frac{1}{4(n-1)} .
$$

The constraints on $p_{1}, q_{1}, p_{2}, q_{2}$ in (114)-(115) are clearly fulfilled, for $n>2$. Pick now any $s>\frac{n}{2}-\frac{3}{4}-\frac{1}{4(n-1)}$ and $\varepsilon>0$ such that $4 \varepsilon<s-\left(\frac{n}{2}-\frac{3}{4}-\frac{1}{4(n-1)}\right)$. We next pick $s_{1} \in\left(\sigma_{1}, \sigma_{1}+\varepsilon\right), s_{2} \in\left(\sigma_{2}, \sigma_{2}+\varepsilon\right)$, $b_{1} \in\left(1-\frac{1}{p_{1}}-\frac{1}{q_{1}}, \frac{1}{2}\right)$, and $b_{2}<\frac{1}{2}$ so that (87) holds. Then (64) follows with $b=\sup \left\{b_{1}, b_{2}\right\}$.

(iii) $\alpha=1$

In this case, we have with $p_{1}=q_{1}=3$

$$
\Sigma \leq c|| u_{1}\left\|_{X_{s, b_{1}}}\right\| u_{2}\left\|_{X_{3 s_{1}+\varepsilon, b_{1}}}\right\| v \|_{X_{-s, b_{1}}}
$$

provided that (114) is satisfied, i.e.

$$
s_{1}>\sigma_{1}=\frac{n-2}{6}, \quad b_{1}>\frac{1}{3}
$$

Therefore, if $s>\frac{n}{2}-1$, taking $\varepsilon>0$ such that $4 \varepsilon<s-\left(\frac{n}{2}-1\right), s_{1} \in\left(\sigma_{1}, \sigma_{1}+\varepsilon\right)$, and $b=b_{1} \in\left(\frac{1}{3}, \frac{1}{2}\right)$, we conclude that

$$
\Sigma \leq c|| u_{1}\left\|_{X_{s, b}}\right\| u_{2}\left\|_{X_{s, b}}\right\| v \|_{X_{-s, b}}
$$

and (64) follows.

\subsection{Proof of Proposition 3.14.}

We begin with the proof of (77) by following closely [17]. Note, however, that the main concern here is to have the condition $s+2 b<1 / 2$ fulfilled. Let $s, b$ be as in the statement of Proposition 3.14, and let $v_{1}, v_{2} \in X_{s, b}$ be decomposed as

$$
v_{i}(x, t)=\int_{\mathbb{R}} \sum_{k \in \mathbb{Z}^{2}} \mathcal{F} v_{i}(k, \tau) e^{i(k \cdot x+\tau t)} d \tau \quad i=1,2 .
$$

(Here, we use the symbol $\mathcal{F}$ instead of $\hat{x}$ to denote Fourier transform in space and time.) Let

$$
f_{i}(k, \tau)=\langle k\rangle^{s}\left\langle\tau-|k|^{2}\right\rangle^{b} \mathcal{F} \bar{v}_{i}(k, \tau), \quad i=1,2 .
$$


Then

$$
\left\|\bar{v}_{1} \bar{v}_{2}\right\|_{X_{s, b^{\prime}}}=\left\|\langle k\rangle^{s}\left\langle\tau+|k|^{2}\right\rangle^{b^{\prime}} \int_{\tau_{1}+\tau_{2}=\tau} \sum_{k_{1}+k_{2}=k} \prod_{i=1}^{2}\left\langle k_{i}\right\rangle^{-s}\left\langle\tau_{i}-\left|k_{i}\right|^{2}\right\rangle^{-b} f_{i}\right\|_{L_{k, \tau}^{2}}
$$

where $\int_{\tau_{1}+\tau_{2}=\tau} \sum_{k_{1}+k_{2}=k}$ stands for $\int_{\mathbb{R}} d \tau_{1} \sum_{k_{1} \in \mathbb{Z}^{2}}$ with the relations $\tau_{1}+\tau_{2}=\tau$ and $k_{1}+k_{2}=k$ satisfied. Let $A_{0}$ (resp. $\left.A_{i}, i=1,2\right)$ denote the region where the largest number among $\left\langle\tau+|k|^{2}\right\rangle,\left\langle\tau_{1}-\left|k_{1}\right|^{2}\right\rangle$ and $\left\langle\tau_{2}-\left|k_{2}\right|^{2}\right\rangle$, is $\left\langle\tau+|k|^{2}\right\rangle$ (resp. $\left.\left\langle\tau_{i}-\left|k_{i}\right|^{2}\right\rangle, i=1,2\right)$. We infer from the relation

$$
\tau+|k|^{2}-\sum_{i=1}^{2}\left(\tau_{i}-\left|k_{i}\right|^{2}\right)=|k|^{2}+\sum_{i=1}^{2}\left|k_{i}\right|^{2}
$$

that

$$
\langle k\rangle^{2}+\sum_{i=1}^{2}\left\langle k_{i}\right\rangle^{2} \leq C\left(\left\langle\tau+|k|^{2}\right\rangle+\sum_{i=1}^{2}\left\langle\tau_{i}-\left|k_{i}\right|^{2}\right\rangle\right)
$$

Let us begin with the region $A_{0}$. (119) gives, with $0<\varepsilon<\inf \left\{\frac{1}{2}\left(\frac{1}{2}-|s|\right), 2(b-|s|)\right\}$ and $-b^{\prime}:=\frac{1}{2}\left(\frac{1}{2}-s\right)+\varepsilon<\frac{1}{2}$

$$
\langle k\rangle^{\frac{1}{2}+s} \prod_{i=1}^{2}\left\langle k_{i}\right\rangle^{-s+\varepsilon} \leq C\left\langle\tau+|k|^{2}\right\rangle^{-b^{\prime}}
$$

The contribution in (118) due to $A_{0}$ is therefore bounded by

$$
\begin{aligned}
& \left.C||\langle k\rangle^{-\frac{1}{2}} \int_{\tau_{1}+\tau_{2}=\tau} \sum_{k_{1}+k_{2}=k}\left\langle k_{i}\right\rangle^{-\varepsilon}\left\langle\tau_{i}-\left|k_{i}\right|^{2}\right\rangle^{-b}\left|f_{i}\right|\right|_{L_{k, \tau}^{2}} \\
& =C||\langle k\rangle^{-\frac{1}{2}} \int_{\tau_{1}+\tau_{2}=\tau} \sum_{k_{1}+k_{2}=k}\left\langle k_{i}\right\rangle^{s-\varepsilon} \mid \mathcal{F} \bar{v}_{i} \|_{L_{k, \tau}^{2}} \\
& =C \|\left.\prod_{i=1}^{2} J^{s-\varepsilon} \mathcal{F}^{-1}\left|\mathcal{F} \bar{v}_{i}\right|\right|_{L_{t}^{2} H_{x}^{-\frac{1}{2}}} \\
& \leq\left. C|| \prod_{i=1}^{2} J^{s-\varepsilon} \mathcal{F}^{-1}\left|\mathcal{F} \bar{v}_{i}\right|\right|_{L_{t}^{2} L_{x}^{q}}, \quad q>\frac{4}{3} \\
& \leq C \prod_{i=1}^{2}\left\|J^{s-\varepsilon} \mathcal{F}^{-1}\left|\mathcal{F} \bar{v}_{i}\right|\right\|_{L_{t}^{4} L_{x}^{2 q},} \quad q>\frac{4}{3} \\
& \leq C \prod_{i=1}^{2}\left\|J^{s-\varepsilon} \mathcal{F}^{-1}\left|\mathcal{F} \bar{v}_{i}\right|\right\|_{X_{\varepsilon, b}^{-}} \\
& \leq C \prod_{i=1}^{2}\left\|v_{i}\right\|_{X_{s, b}}
\end{aligned}
$$

where we used the fact that $L^{q}\left(\mathbb{T}^{2}\right) \subset H^{-\frac{1}{2}}\left(\mathbb{T}^{2}\right)$ for $q>4 / 3$ (by dualizing the Sobolev embedding $H^{\frac{1}{2}}\left(\mathbb{T}^{2}\right) \subset$ $L^{p}\left(\mathbb{T}^{2}\right)$ for $\left.p<4\right)$, Hölder inequality, and (87)-(88). We also used the notation

$$
\|u\|_{X_{s, b}^{-}}=\left(\int_{\mathbb{R}} \sum_{k \in \mathbb{Z}^{2}}\langle k\rangle^{2 s}\left\langle\tau-|k|^{2}\right\rangle^{2 b}|\mathcal{F} u(k, \tau)|^{2} d \tau\right)^{\frac{1}{2}}=\|\bar{u}\|_{X_{s, b}}
$$

borrowed from [16]. It remains to estimate the contributions in (118) due to the regions $A_{1}$ and $A_{2}$. By symmetry, we can consider only the region $A_{1}$. In $A_{1}$, since $-s+\frac{\varepsilon}{2}<b$, we have that

$$
\left\langle k_{2}\right\rangle^{-s+\varepsilon}\left\langle k_{1}\right\rangle^{-s} \leq C\left\langle\tau_{1}-\left|k_{1}\right|^{2}\right\rangle^{-s+\frac{\varepsilon}{2}} \leq C\left\langle\tau_{1}-\left|k_{1}\right|^{2}\right\rangle^{b}
$$

and therefore the contribution in (118) is bounded by

$$
\left.\left\|\langle k\rangle^{s}\left\langle\tau+|k|^{2}\right\rangle^{b^{\prime}} \int_{\tau_{1}+\tau_{2}=\tau} \sum_{k_{1}+k_{2}=k}\left|f_{1}\right|\left\langle k_{2}\right\rangle^{-\varepsilon}\left\langle\tau_{2}-\left|k_{2}\right|^{2}\right\rangle^{-b}\left|f_{2}\right|\right\|\right|_{L_{k, \tau}^{2}}=C|| \mathcal{F}^{-1}\left|f_{1}\right| J^{s-\varepsilon} \mathcal{F}^{-1}\left|\mathcal{F} \bar{v}_{2}\right| \| X_{s, b^{\prime}}
$$


By (86)-(87) with $-s>1 / 3$ and $-b^{\prime}$ chosen sufficiently close to $\frac{1}{2}$, we have that

$$
X_{-s,-b^{\prime}} \subset L^{6}\left(\mathbb{R} ; L^{6}\left(\mathbb{T}^{2}\right)\right), \quad \text { hence } \quad L^{\frac{6}{5}}\left(\mathbb{R} ; L^{\frac{6}{5}}\left(\mathbb{T}^{2}\right)\right) \subset X_{s, b^{\prime}}
$$

It follows that

$$
\begin{aligned}
\left\|\mathcal{F}^{-1}\left|f_{1}\right| J^{s-\varepsilon} \mathcal{F}^{-1}\left|\mathcal{F} \bar{v}_{2}\right|\right\| X_{s, b^{\prime}} & \leq\left. C\left\|\mathcal{F}^{-1}\left|f_{1}\right| J^{s-\varepsilon} \mathcal{F}^{-1}\left|\mathcal{F} \bar{v}_{2}\right|\right\|\right|_{L_{t}^{\frac{6}{5}} L_{x}^{\frac{6}{5}}} \\
& \leq\left.\left. C\left\|\mathcal{F}^{-1}\left|f_{1}\right|\right\|\right|_{L_{t}^{2} L_{x}^{2}}\left\|J^{s-\varepsilon} \mathcal{F}^{-1}\left|\mathcal{F} \bar{v}_{2}\right|\right\|\right|_{L_{t}^{3} L_{x}^{3}} \\
& \leq C\left\|\bar{v}_{1}\right\|_{X_{s, b}^{-}}\left\|J^{s-\varepsilon} \mathcal{F}^{-1}\left|\mathcal{F} \bar{v}_{2}\right|\right\|_{X_{\varepsilon, b}^{-}} \\
& \leq C\left\|v_{1}\right\|_{X_{s, b}}\left\|v_{2}\right\|_{X_{s, b}}
\end{aligned}
$$

where we used Hölder inequality and (87)-(88) with $p=q=3$. This completes the proof of 777$)$.

To derive (78) from (77), we consider two functions $u_{1}, u_{2}$ in $X_{0, b}(\Omega) \subset X_{s, b}(\Omega)$, and consider their odd extensions $v_{1}, v_{2}$ to $(-\pi, \pi)^{2}$; i.e., $v_{i}\left(\epsilon_{1} x_{1}, \epsilon_{2} x_{2}\right)=\epsilon_{1} \epsilon_{2} u_{i}\left(x_{1}, x_{2}\right)$ for $x=\left(x_{1}, x_{2}\right) \in \Omega$ and $\epsilon_{i}= \pm 1$. Note that $v_{1}, v_{2} \in X_{0, b}$ and that $\bar{u}_{1} \bar{u}_{2}=\left(\bar{v}_{1} \bar{v}_{2}\right)_{\left.\right|_{\Omega}}$. For any function $w=\sum_{k \in \mathbb{N}^{2}} \int_{\mathbb{R}} \mathcal{F} w(k, \tau) e^{i \tau t} \cos \left(k_{1} x_{1}\right) \cos \left(k_{2} x_{2}\right) d \tau$, we set

$$
\|w\|_{X_{s, b}(\Omega)_{N}}^{2}=\sum_{k \in \mathbb{N}^{2}} \int_{\mathbb{R}}\left\langle\tau+|k|^{2}\right\rangle^{2 b}\langle k\rangle^{2 s}|\mathcal{F} w(k, \tau)|^{2} d \tau .
$$

The Bourgain space $X_{s, b}(\Omega)_{N}$ (with Neumann boundary conditions) is defined as the space of the w's for which the norm $\|w\|_{X_{s, b}(\Omega)_{N}}$ is finite. Since the function $\bar{v}_{1} \bar{v}_{2}$ is even with respect to both $x_{1}$ and $x_{2}$, we have that

$$
\left\|\bar{u}_{1} \bar{u}_{2}\right\|_{X_{s, b^{\prime}}(\Omega)_{N}} \sim C\left\|\bar{v}_{1} \bar{v}_{2}\right\|_{X_{s, b^{\prime}}} \leq C\left\|v_{1}\right\|_{X_{s, b}}\left\|v_{2}\right\|_{X_{s, b}} \leq C\left\|u_{1}\right\|_{X_{s, b}(\Omega)}\left\|u_{2}\right\|_{X_{s, b}(\Omega)}
$$

We claim that $X_{s, b}(\Omega)=X_{s, b}(\Omega)_{N}$ for $|s|<1 / 2$ and $|b| \leq 1$. Note first that this is true for $|s|<\frac{1}{2}$ and $b=0$, since

$$
X_{s, 0}(\Omega)=L^{2}\left(\mathbb{R} ; H^{s}(\Omega)\right)=X_{s, 0}(\Omega)_{N} .
$$

The claim is also true for $|s|<1 / 2$ and $b=1$, since

$$
u \in X_{s, 1}(\Omega) \Longleftrightarrow u \in X_{s, 0}(\Omega) \text { and } i u_{t}+\Delta u \in X_{s, 0}(\Omega)
$$

and since a similar criterion may be written for $X_{s, 1}(\Omega)_{N}$. The claim is also true for $|s|<1 / 2$ and $0 \leq b \leq 1$ by interpolation, and for $|s|<1 / 2$ and $|b| \leq 1$ by duality. (78) follows for $u_{1}, u_{2} \in X_{0, b}(\Omega)$, and also for $u_{1}, u_{2} \in X_{s, b}(\Omega)$ by density. This completes the proof of Proposition 3.14 .

\section{References}

[1] Ball, J.M., Marsden, J.E., Slemrod, M., Controllability for distributed bilinear systems, SIAM J. Control and Optim. 20, 1982.

[2] Baudouin, L., Kavian, O., Puel, J.-P., Regularity for a Schrödinger equation with singular potential and application to bilinear optimal control, J. of Differential Equations 216 (2005), 188-222.

[3] Baudouin, L., Puel, J.-P., Uniqueness and stability in an inverse problem for the Schrödinger equation, Inverse Problems 18 (2001), 1537-1554.

[4] Baudouin, L., Salomon, J., Constructive solutions of a bilinear control problem for a Schrödinger equation, Systems and Control Letters, 57 (2008), 453-464.

[5] Beauchard, K., Local Controllability of a 1-D Schrödinger equation, J. Math. Pures et Appl. 84 (2005), 851-956.

[6] Beauchard, K., Coron, J.-M., Controllability of a quantum particle in a moving potential well, J. Functional Analysis 232 (2006), 328-389. 
[7] Boscain, U., Charlot, G., Gauthier, J.-P., Optimal control of the Schrödinger equation with two or three levels, Nonlinear and adaptive control (Sheffield, 2001), volume 281. Lecture Notes in Control and Inform. Sci., Springer, Berlin, 2003.

[8] Bourgain, J., Fourier transform restriction phenomena for certain lattice subsets and applications to non-linear evolution equations, part I: Schrödinger equations, Geom. \& Funct. Anal. 3 (1993), 107 156.

[9] Bourgain, J., Global Solutions of Nonlinear Schrödinger Equations, Colloqium Publication, Vol. 46, American Mathematical Society, 1999.

[10] Cancès, E., Le Bris, C., Pilot, M., Contrôle optimal bilinéaire d’une équation de Schrödinger, CRAS Paris 330 (2000), 567-571.

[11] Cardoulis, L., Gaitan, P., Simultaneous Identification of the Diffusion Coefficient and the Potential for the Schrödinger Operator with only one Observation, arXiv:0911.3300

[12] Cardoulis, L., Cristofol, M., Gaitan, P., Inverse Problem for the Schrödinger Operator in an Unbounded Strip, Journal of Inverse and Ill-posed Problems, 16 (2008), 127-146.

[13] Dehman, B., Gérard, P., Lebeau, G., Stabilization and control for the nonlinear equation on a compact surface, Math. Z 254(2006), 729-749.

[14] Dolecki S., Russell D.L., A general theory of observation and control, SIAM J. Control Optimization 15 (1977), 185-220.

[15] Glass, O., Guerrero, S., Some exact controllability results for the linear KdV equation and uniform controllability in the zero-dispersion limit, Asymptotic Analysis 60 (2008), 61-100.

[16] Grünrock, A., On the Cauchy- and periodic boundary value problem for a certain class of derivative nonlinear Schrödinger equations, arXiv:math/0006195v1 (2000).

[17] Grünrock, A., Some local wellposedness results for nonlinear Schrödinger equations below $L^{2}$, arXiv:math.AP/0011157v2 (2001).

[18] Hörmander, L., The Analysis of Linear Partial Differential Operators. I. Distribution Theory and Fourier Analysis. Reprint of the second (1990) edition. Classics in Mathematics. Springer-Verlag, Berlin, 2003.

[19] Illner, R., Lange, H., Teismann, H., A note on the exact internal control of nonlinear Schrödinger equations, CRM Proceedings and Lecture Notes 33 (2003), 127-137.

[20] Illner, R., Lange, H., Teismann, H., Limitations on the control of Schrödinger equations, ESAIM Control Optim. Calc. Var., 12 (2006), 615-635.

[21] Jaffard, S., Contrôle interne des vibrations d'une plaque rectangulaire, Portugal Math. 47 (4), 423-429 (1990).

[22] Kenig, C.E., Ponce, G., Vega, L., Quadratic forms for the 1-D semilinear Schrödinger equation, Transactions of the AMS 348 (1996), 3323-3353.

[23] Komornik, V., On the zeros of Bessel type functions and applications to exact controllability problems, Asymptotic Anal. 5 (1991), 115-128.

[24] Komornik, V., Loreti, P., Fourier series in control theory, Springer Monographs in Mathematics, Springer-Verlag, New York, 2005.

[25] Lange, H., Teismann, H., Controllability of nonlinear Schrödinger equation in the vicinity of the ground state, Mth.meth. Appl. Sci., (in press), 2007.

[26] Lasiecka, I., Triggiani, R., Optimal regularity, exact controllability and uniform stabilization of Schrödinger equaitons with Dirichlet control, Diff. Integral Eq. 5 (1992), 521-535.

[27] Lasiecka, I., Triggiani, R., Zhang, X., Carleman estimates at the $H^{1}(\Omega)$-and $L_{2}(\Omega)$-level for nonconservative Schrödinger equations with unobserved Neumann B.C., Arch. Inequal. Appl. 2 (2004), no. 2-3, $215-338$. 
[28] Laurent, C. Global controllability and stabilization for the nonlinear Schrödinger equation on an interval, ESAIM Control Optim. Calc. Var. (2009), DOI: 10.1051/cocv/2009001

[29] Laurent, C. Global controllability and stabilization for the nonlinear Schrödinger equation on some compact manifolds of dimension 3, arXiv:0903.1737 (March 2009)

[30] Laurent, C., Rosier, L., Zhang, B.-Y., Control and Stabilization of the Korteweg-de Vries Equation on a periodic domain, arXiv:0905.3478 (May 2009)

[31] Lebeau, G., Contrôle de l'équation de Schrödinger, J. Math. Pures Appl. 71 (1992), 267-792.

[32] Linares, F., Ortega, J.H., On the controllability and stabilization of the linearized Benjamin-Ono equation, ESAIM Control Optim. Calc. Var. 11 (2005), no. 2, 204-218.

[33] Linares, F., Ponce, G., Introduction to Nonlinear Dispersive Equations, Publicações Matemáticas, IMPA, 2006.

[34] Lions, J.-L., Contrôlabilité exaCte, Perturbations et stabilisation de Systèmes DistribuÉs, Tome 1, Masson, Paris, 1988.

[35] Lions, J.-L., Magenes, E., Problèmes Aux Limites non Homogènes et Applications, Vol. 1, Dunod, Paris, 1968.

[36] Liu, K., Locally distributed control and damping for the conservative systems, SIAM J. Cont. Optim., 35 (1997), 1574-1590.

[37] Machtyngier, E., Exact controllability for the Schrödinger equation, SIAM J. Control and Optimization, 32 (1994), 24-34.

[38] Machtyngier, E., Zuazua, E., Stabilization of the Schrödinger equation, Portugaliae Matematica 51 (1994), 243-256.

[39] Mercado, A., Osses, A., Rosier, L., Inverse problems for the Schrödinger equation via Carleman inequalities with degenerate weights, Inverse Problems 24 (2008), 015017 (18pp).

[40] Miller, L., How violent are fast controls for Schrödinger and plate vibrations? Arch. Ration. Merch. Anal. 172 (2004), 429-456.

[41] Mirrahimi, M., Rouchon, P., Turinici, G., Lyapounov control of bilinear Schrödinger equations, Automatica 41 (2005), 1987-1994.

[42] Phung, K.-D., Observability and controllability for Schrödinger equations, SIAM J. Control Optm. 40 (2001), 211-230.

[43] Ramdani, K., Takahashi, T., Tenenbaum, G., Tucsnak, M., A spectral approach for the exact observability of infinite-dimensional systems with skew-adjoint generator, Journal of Functional Analysis, 226 (2005) 193-229.

[44] Rosier, L., Exact boundary controllability for the Korteweg-de Vries equation on a bounded domain, ESAIM Control Optim. Calc. Var. 2 (1997), 33-55.

[45] Rosier, L., Zhang, B.-Y., Null controllability of the complex Ginzburg-Landau equation, Ann. Inst. H. Poincaré Anal. Non Linéaire 26 (2009), no. 2, 649-673.

[46] Rosier, L., Zhang, B.-Y., Local exact controllability and stabilizability of the nonlinear Schrödinger equation on a bounded domain, SIAM J. Control Optim. 48 (2009), no. 2, 972-992.

[47] Rosier, L., Zhang, B.-Y., Exact boundary controllability of the nonlinear Schrödinger equation, J. Differential Equations 246 (2009), no. 10, 4129-4153.

[48] Russell, D.L., Zhang, B.-Y., Exact controllability and stabilizability of the Korteweg-de Vries equation, Trans. Amer. Math. Soc., 348 (1996), 3643-3672.

[49] Simon, J.,Compact sets in the space $L^{p}(0, T ; B)$, Annali di Matematica pura ed applicata (IV), vol. CXLVI (1987), 65-96. 
[50] Tao, T., Nonlinear dispersive equations. Local and global analysis, CBMS Regional Conference Series in Mathematics, 106, Providence, 2006. xvi+373 pp.

[51] Yuan, G., Yamamoto, M., Carleman estimates for the Schrödinger equation and applications to an inverse problem and an observability inequality, preprint.

[52] Zuazua, E., Remarks on the controllability of the Schrödinger equation, Quantum control: mathematical and numerical challenges, 193-211, CRM Proc. Lecture Notes, 33, Amer. Math. Soc., Providence, RI, 2003. 